\title{
Numerical Investigation of a Novel RBCC Ejector Configuration Compared to a Traditional Circular Ejector
}

by

\section{Adrian Gerber}

\author{
A Thesis submitted to \\ the Faculty of Graduate Studies and Research \\ in partial fulfilment of \\ the requirements for the degree of \\ Master of Applied Science \\ in
}

\author{
Mechanical and Aerospace Engineering \\ Carleton University \\ Ottawa, Ontario, Canada
}

March 2015

Copyright (c)

2015 - Adrian Gerber 


\section{Abstract}

The rocket based combined cycle can be an alternative engine to power a vehicle into space. The engine has 4 stages and one of the more challenging stages to increase performance is the first stage, the ejector stage. One proposed method to increase performance is by achieving higher entrainment performance and mixing at low free-stream velocities. The Exchange Inlet is used as an alternative nozzle to achieve this performance. The Exchange Inlet is compared to a conical nozzle within a RBCC engine with computational fluid dynamics at various pressures. The mixing and entrainment properties are compared between the two engine configurations and additional cases are investigated with shorter mixing sections to further investigate these properties. The Exchange Inlet is found to have better entrainment than the circular nozzle in all cases. 
For my mother, who always pushed me to never give up. 


\section{Acknowledgments}

I'd like to thank the Japanese Space Agency for providing the software and expertise required to take on the project. I would also like to thank my professor for patiently pushing, motivating, and always showing me a little bit of light and knowledge at the end of every turn. Lastly I would like to thank my family, friends, and girlfriend especially for the constant support and patience. 


\section{Table of Contents}

Abstract ii

Acknowledgments iv

List of Tables $\quad$ vii

List of Figures viii

List of Acronyms xii

List of Symbols $\quad$ xiv

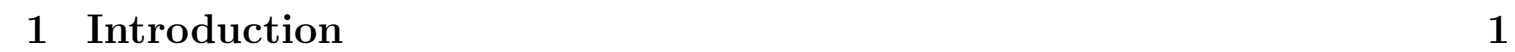

1.1 Background . . . . . . . . . . . . . . . . . . . 1

1.2 Rocket Based Combined Cycle . . . . . . . . . . . . . . . . . 4

1.3 Ejectors . . . . . . . . . . . . . . . . . . . . 6

1.4 Exchange Inlet . . . . . . . . . . . . . . . . . . . 11

1.5 Problem Statement . . . . . . . . . . . . . . . . . . . . . . . 13

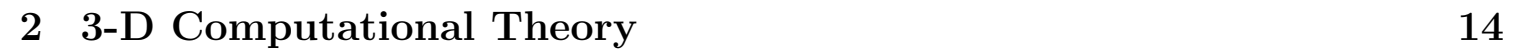

$2.1 \quad$ Discretization of Domain . . . . . . . . . . . . . . . . 15

2.2 Governing Equations . . . . . . . . . . . . . . . . 16

2.3 Favre-Averaged Navier-Stokes . . . . . . . . . . . . . . . . . . . . . . 18 
2.4 Turbulence Model . . . . . . . . . . . . . . . . . . . . . . . . . . . . . . . 25

2.5 Kodera . . . . . . . . . . . . . . . . . . . . . . . . . . . 28

\begin{tabular}{llr}
3 & Methodology & 29 \\
\hline
\end{tabular}

3.1 Geometry . . . . . . . . . . . . . . . . . . . . . . 29

3.1.1 $\quad$ Twin Circular Configuration . . . . . . . . . . . . . . . . . . . 29

3.1 .2 Exchange Inlet Geometry . . . . . . . . . . . . . . . . . 31

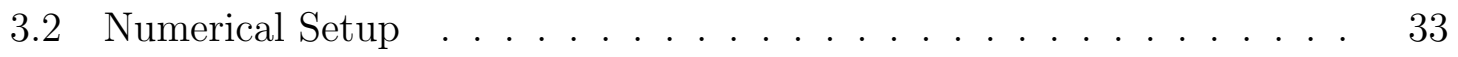

$3.2 .1 \quad$ Domain and Boundary Conditions . . . . . . . . . . . . . . . 33

3.2 .2 Initialization . . . . . . . . . . . . . . . . . . . . 34

3.2 .3 Residuals . . . . . . . . . . . . . . . . . . . 35

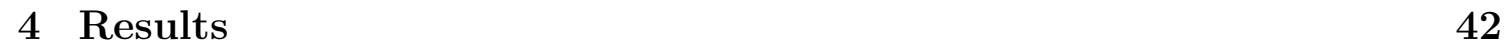

4.1 Validation . . . . . . . . . . . . . . . . . . . . . . . . . . . 42

4.2 Full Engine Configuration . . . . . . . . . . . . . . . . . 45

4.2 .1 Low Pressure Cold Gas Configuration . . . . . . . . . . . . . . 45

$4.2 .2 \quad$ High Pressure Cold Gas Configuration . . . . . . . . . . . . . 58

4.3 Half Engine Configuration . . . . . . . . . . . . . . . . . 70

$4.3 .1 \quad$ Low Pressure Cold Gas . . . . . . . . . . . . . . . . . . . . . . 70

4.3 .2 High Pressure Cold Gas . . . . . . . . . . . . . . . . . . . 80

4.4 Additional Experimental Data . . . . . . . . . . . . . . . . . 87

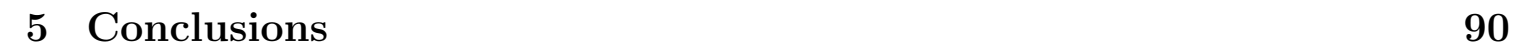

\begin{tabular}{ll}
\hline List of References & 92
\end{tabular} 


\section{List of Tables}

$3 \quad$ RNEP Area Properties $\ldots \ldots \ldots$. . . . . . . . . . . . . . . . . . . . 33

$4 \quad$ Rocket Nozzle Stagnation Conditions . . . . . . . . . . . . . . . 35

$5 \quad$ Grid Sizing $\ldots \ldots \ldots \ldots \ldots \ldots$

$6 \quad$ Summary Of Low Pressure configuration Results. . . . . . . . . . 57

7 Summary Of High Pressure Configuration Results . . . . . . . . . . 69

8 Summary Of Low Pressure Short and Long Configuration Results . . 79

$9 \quad$ Summary Of High Pressure Short Configuration Results _. . . . . . 88 


\section{List of Figures}

$1 \quad$ NASA Budget since 1954 (recreated from NASA Data $[4]$ ) . . . . . . 2

$2 \quad$ Specific Impulse of Various Technologies (taken from $[10 \mid$ data recre-

ated and taken from $[11]$. . . . . . . . . . . . . . . . 3

$3 \quad$ Rocket Flow path $[10]$. . . . . . . . . . . . . . . . . . 4

$4 \quad$ RBCC Engine modes $[10]$. . . . . . . . . . . . . . . . . . . . 5

5 Fabri choke . . . . . . . . . . . . . . . . . . . . . . . . . . . . 7

6 Saturated Supersonic Flow . . . . . . . . . . . . . . . . . . . . 8

$7 \quad$ Features of a strutjet configuration in a ramjet engine $[39]$. . . . . . 11

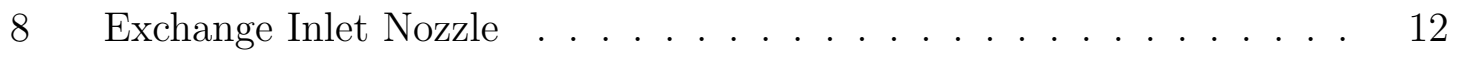

$9 \quad$ Cell Centered FVM volume centered FVM . . . . . . . . . . . . . . . 15

10 Twin Circular configuration 3D view . . . . . . . . . . . . . . . . . . 30

11 Twin Circular RNEP . . . . . . . . . . . . . . . . . . . . . . . . . . . 31

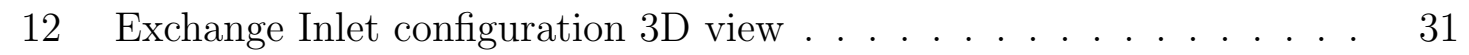

13 Exchange Inlet configuration RNEP . . . . . . . . . . . . . . . . . . . 32

14 Domain of Exchange Inlet configuration . . . . . . . . . . . . . . . . 34

15 Exchange Inlet configuration Mass flow Residuals . . . . . . . . . . . 36

$16 \quad$ Exchange Inlet configuration local residuals $\quad$. . . . . . . . . . . . . . 37

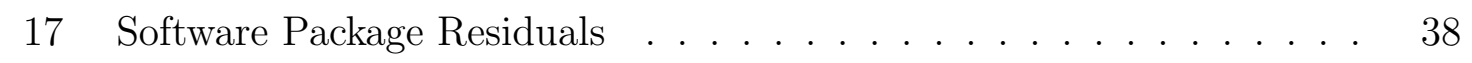

18 Medium Grid within the Exchange Inlet . . . . . . . . . . . . . . . . 39

19 Medium grid near Exchange inlet nozzle . . . . . . . . . . . . . . . . 39 
20 Grid Refinement Study (Pressure) $\ldots \ldots \ldots$. . . . . . . . . . 40

21 Grid Refinement Study (Velocity) . . . . . . . . . . . . . . . . . . 41

22 Exchange inlet lower surface pressure plot at Rocket Pressure $868 \mathrm{kPa} \quad 43$

$23 \quad$ Twin circular pressure plot along lower surface . . . . . . . . . . . 44

24 Lower Surface Low Pressure Distribution for Long Engine configuration 46

$25 \quad$ Upper Surface Low Pressure Distribution for Long Engine configuration 47

26 Low Rocket Chamber Pressure RNEP Mach Contours . . . . . . . . . 49

27 Low Rocket Chamber Pressure Mach Contours at ESP . . . . . . . 50

28 Low Rocket Chamber Pressure Mach Contours at EEP . . . . . . . . 52

29 Low Rocket Chamber Pressure $\mathrm{O}_{2}$ Partial Density at EEP . . . . . . 54

$30 \quad$ Stream Traces along Rocket Flow Plane of the Exchange Inlet $(28 \mathrm{~mm}$

offset of the symmetry Plane) $\ldots \ldots \ldots \ldots \ldots$

31 Twin Circular Stream Trace . . . . . . . . . . . . . . . . . 55

32 Exchange Inlet Rocket Flow Plane Pressure Plot . . . . . . . . . . . 56

33 Twin Circular Rocket Flow Plane Pressure Plot . . . . . . . . . . . 56

34 Exchange Inlet $\mathrm{O}_{2}$ Mass Fraction along Rocket Flow Plane . . . . . . 57

35 Twin Circular $\mathrm{O}_{2}$ Partial Density along Rocket flow Plane . . . . . 57

36 Lower Surface High Pressure Distribution for Long Engine Configuration 58

$37 \quad$ Upper Surface High Pressure Distribution for Long Engine configuration 59

38 High Rocket Chamber Pressure Mach Contours at RNEP . . . . . . . 61

39 High Rocket Chamber Pressure Mach Distribution at ESP . . . . . . 62

40 High Rocket Chamber Pressure Mach Distribution at EEP . . . . . . 64

41 High Rocket Chamber Pressure $\mathrm{O}_{2}$ Partial Density at EEP . . . . . . 65

42 Exchange Inlet configuration Stream Trace High Pressure $(28 \mathrm{~mm}$ offset of symmetry plane) $\ldots \ldots \ldots \ldots \ldots$

$43 \quad$ Twin circular High Pressure Stream trace. . . . . . . . . . . . . 67

44 Exchange Inlet configuration Pressure Distribution $\ldots \ldots \ldots$ 
45 Twin circular configuration Engine Rocket Flow plane Pressure Plot . 68

46 Exchange Inlet $\mathrm{O}_{2}$ Partial Density Rocket flow plane . . . . . . . . . 68

47 Twin circular configuration Engine Outlet $\mathrm{O}_{2}$ Partial Density. . . . . 69

48 Lower Surface Low Pressure Distribution for Short Engine configuration 71

49 Upper Surface low Pressure Distribution for short Engine configuration 72

50 Short Configuration Low Rocket Chamber Pressure Mach Contours at RNEP . . . . . . . . . . . . . . . . . . . . . 73

51 Short Configuration Low Rocket Chamber Pressure Mach Distribution at EEP . . . . . . . . . . . . . . . . . . . . . . 75

52 Short Configuration Low Rocket Chamber Pressure $\mathrm{O}_{2}$ Partial Density at EEP . . . . . . . . . . . . . . . . . . 76

53 Exchange Inlet Short Engine Configuration Stream Trace . . . . . . . 77

54 Exchange Inlet short engine $\mathrm{O}_{2}$ Partial Density planar view . . . . . . 78

55 Twin circular Short Configuration Low Pressure Rocket Centered Stream Trace . . . . . . . . . . . . . . . . . . . . . . . 78

56 Twin circular Short Engine Rocket Flow Plane $\mathrm{O}_{2}$ Partial Density . . $\quad 79$

57 Lower Surface High Pressure Distribution for Short Engine configuration 80

58 Upper Surface High Pressure Distribution for Short Engine configuration 81

59 Short Configuration High Rocket Chamber Pressure Mach Distributions at RNEP . . . . . . . . . . . . . . . . . . . . . . . . . . . . . . . 82

60 Short Configuration High Rocket Chamber Pressure Mach Distribution at EEP . . . . . . . . . . . . . . . . . . . . . . . 84

61 Short Configuration High Rocket Chamber Pressure $\mathrm{O}_{2}$ Partial Density at EEP . . . . . . . . . . . . . . . . . . . . . 85

62 Exchange Inlet Short configuration Stream Trace . . . . . . . . . . . 86

63 Twin Short Engine configuration Stream Trace. . . . . . . . . . . . . 86 
64 Exchange Inlet Short configuration $\mathrm{O}_{2}$ Partial Density along rocket flow plane . . . . . . . . . . . . . . . . . . 87

65 Twin Short configuration $\mathrm{O}_{2}$ Partial Density along Rocket Plane . . . 87

66 Exchange Inlet Short configuration Lower surface low pressure Experimental VS Numerical data . . . . . . . . . . . . . . . . . . . . 88

67 Twin circular Short configuration Lower surface low pressure Experimental VS Numerical data . . . . . . . . . . . . . . . . . . . . . . . . 89 


\section{List of Acronyms}

\begin{tabular}{ll} 
Acronyms & Definition \\
\hline \hline CFD & Computational Fluid Dynamics \\
EEP & Engine Exit Plane \\
FANS & Expanding Section Plane \\
FVM & Finite Volume Method \\
HLLEW & Harten, Lax, Van Leer, Wada Reimann Solver \\
ISP & Specific Impulse \\
JAXA & Japanese Space Agency \\
LUSGS & Lower Upper Symmetric Gauss Seidel Implicit Method \\
NASA & National Aeronatics and Space Administration \\
N-S & Navier Stokes \\
RANS & Reynolds Averaged Navier Stokes \\
RBCC & Rocket Based Combined Cycle
\end{tabular}




$\begin{array}{ll}\text { RNEP } & \text { Rocket Nozzle Exit Plane } \\ \text { Roscosmos } & \text { Russian Federal Space Agency } \\ \text { SSTO } & \text { Single Stage To Orbit }\end{array}$




\section{List of Symbols}

\begin{tabular}{ll}
\hline Symbols & Definition \\
\hline \hline$E$ & Total Energy $[\mathrm{J}]$ \\
$H$ & Total Enthalpy $[\mathrm{J}]$ \\
$I_{S P}$ & Specific Impulse $[\mathrm{s}]$ \\
$P r$ & Prandtl Number \\
$R$ & Specific Gas Constant $[\mathrm{J} / \mathrm{K}$ mol $]$ \\
$T$ & Temperature $[\mathrm{K}]$ \\
$U$ & Velocity $[\mathrm{m} / \mathrm{s}]$ \\
$V$ & Volume $\left[m^{3}\right]$ \\
$c_{p}$ & Specific Heat at constant pressure \\
$c_{v}$ & Specific Heat at constant volume \\
$e$ & Internal Energy per unit mass $[\mathrm{J} / \mathrm{kg}]$ \\
$h$ & Enthalpy per unit mass $[\mathrm{J} / \mathrm{kg}]$ \\
& \\
& \\
& \\
& \\
&
\end{tabular}




\begin{tabular}{|c|c|}
\hline$\dot{m}$ & Mass Flow \\
\hline$q$ & generic variable \\
\hline$q_{j}$ & Heat Flux $\left[\mathrm{W} / m^{2}\right]$ \\
\hline$s$ & cauchy stress tensor \\
\hline$t$ & Time $[\mathrm{s}]$ \\
\hline$u$ & Velocity x component $[\mathrm{m} / \mathrm{s}]$ \\
\hline$v$ & Velocity y component $[\mathrm{m} / \mathrm{s}]$ \\
\hline$p$ & Pressure $[\mathrm{Pa}]$ \\
\hline$x$ & Coordinate \\
\hline$\kappa$ & Thermal Conductivity $[\mathrm{W} / \mathrm{mK}]$ \\
\hline$\rho$ & Density $\left[\mathrm{kg} / \mathrm{m}^{3}\right]$ \\
\hline$\zeta$ & Stokes Hypothesis \\
\hline$\mu$ & dynamic viscosity $[\mathrm{Pa} \mathrm{s}]$ \\
\hline$\tau$ & Shear Tensor \\
\hline$\delta$ & Kroniker delta \\
\hline$\epsilon$ & Venkatakrishnan Limiter \\
\hline$\nu$ & Dynamic viscosity \\
\hline & Subscript \\
\hline$i$ & index $1: 3$ \\
\hline
\end{tabular}


j $\quad$ index 1:3

Superscript

$-\quad$ mean value from Reynolds Averaging
$\sim$
mean values from Favre Averaging
$\quad \quad$ fluctuations from Reynolds Averaging




\section{Chapter 1}

\section{Introduction}

\section{$1.1 \quad$ Background}

Modern rocketry has been in its current form since Robert Goddard first developed the first functional liquid fueled rocket in 1926 [1]. It has been since this first successful small rocket launch that rockets have been in serious development for almost the last century. It became a fascination of many individuals to develop a vehicle that could soar past the sky and into space, including Wernher Von Braun. During World War 2, he developed the famous V2 rocket that was able to fly $300 \mathrm{~km}$ in range and carry a payload of 1,000 kg. After the end of World War 2, rockets and space travel went hand in hand as ways to move beyond our atmosphere were examined. Many projects were developed to find optimal rocket technology under leading rocket men like Werner Van Braun. NASA was founded in 1958 where the beginning of manned space travel finds it's birth. NASA developed rockets under the United States government in competition with the Russian governments Roscosmos to accomplish firsts, such as the first object in space (Sputnik), the first man in space (Yuri Gagarin), and eventually the first man on the moon (Neil Armstrong). All of these were accomplished by the great works in rocket technology under both organizations. While technology development has slowed down since the late 60's due to the financial constraints, 


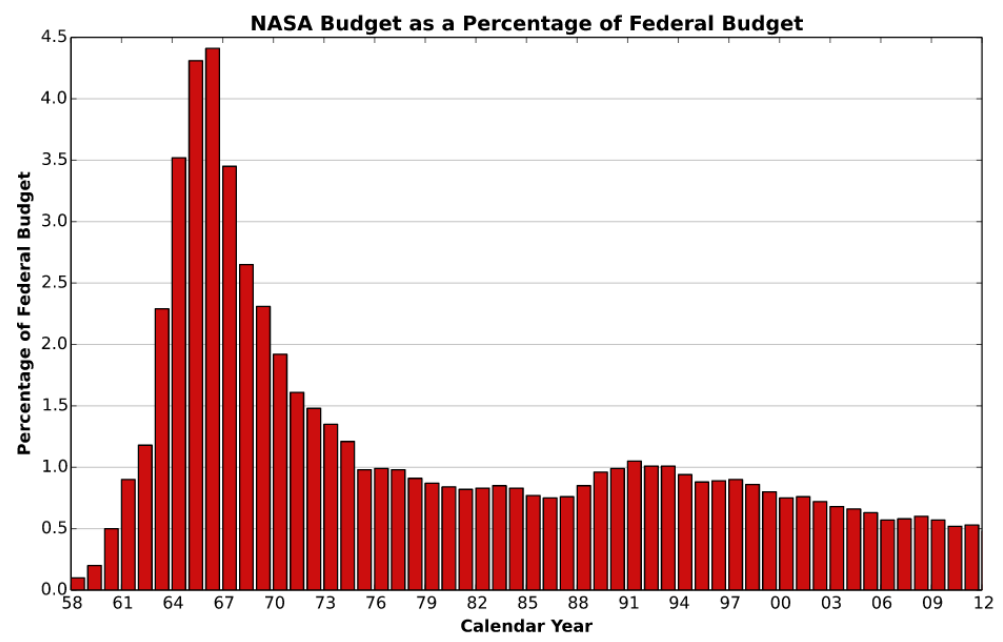

Figure 1: NASA Budget since 1954 (recreated from NASA Data [4])

they have still been underway [2].

The budget of NASA has continually declined over the years in terms of percentage of American budget and represents an example of the expenditure of society on space as shown in the Figure 1. As public interest has dwindled, the budgetary spending on space technology has reflected this quite well. NASA's spending on space research and technology is divided even further between it's respective departments. Of the 17 billion dollars allocated to NASA, 551 million and 705 million dollars are allocated towards aeronautic and space research respectively. For space launches, only $\$ 854$ million is being spent. That amounts to $5 \%$ of the budget being allocated towards any kind of rocket technology. That indicates a major restriction when it comes to buying fuel, resources for research and for rockets. This creates an understandable desire to design, build, and find cheaper rocket technology [3].

The goal of obtaining cheaper access to space can be achieved by designing vehicles with the highest possible specific impulse (ISP). Specific impulse is a measure of an engines ability to transfer energy into thrust [5]. There is a balance between specific impulse and total thrust where the payload weight starts to become a concern. Common payloads include man and satellites and with the world so dependent on 
space technology, reducing costs is important. Currently the common technology is to use rockets to send the desired payload into space. Alternative ideas exist, such as the space elevator and even a rail gun launch system but none have progressed beyond theory [6,7]. Often the rocket designs are not re-usable. There are exceptions to this such as the work being done by SpaceX, Swiss Space Systems and some of the previous NASA vehicles such as the shuttle. Current rocket designs also incorporate multistaging. Multistaging allows one to carry up tanks with oxidizer and fuel and drop them once the tank is empty. This decreases the amount of energy required to send the object into space as excess weight is cut. As examples, the shuttle used to transport man to space as well as the Russian Soyuz require 3 stages in order to reach space. A significant portion of the weight required to launch vehicles into space is due to the requirement to contain both the fuel and oxidizer on board. Escher [8,9] investigates alternatives to the shuttle and identifies a number of potential air breathing designs of which the Rocket Based Combined Cycle (RBCC) shows great promise. The focus of this document will be the rocket based combined cycle.

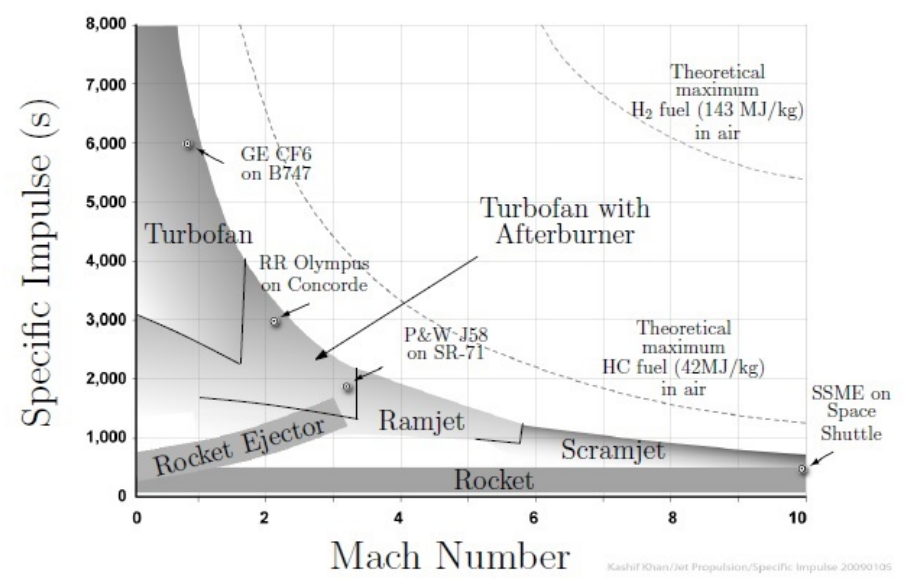

Figure 2: Specific Impulse of Various Technologies (taken from [10] data recreated and taken from [11])

From Figure 2 it can be seen that other engine modes have their advantages 
and disadvantages, the simplicity of the rocket based combined cycle makes it very appealing. In Figure 2 it can be seen that the other methods have their place and even higher efficiency at various flight stages/speeds. The turbine based combined cycle as an example would have a higher initial ISP at low speeds, however suffers from an inability to fly without atmosphere and relies on technology that does not exist yet. The Rocket Based Combined Cycle (RBCC) relies on rocket technology which already exists.

\subsection{Rocket Based Combined Cycle}

The RBCC has been a concept engine for a space plane since at least 1966 [8]. A rocket based combined cycle engine is described as an engine that houses both a rocket engine and is combined with an air breathing engine. This means that rocket flow and air flow are combined into a mixing duct allowing for augmented thrust resulting in a higher specific impulse over traditional rocket engines [12]. The flow is ignited either in the mixing duct or at a different location depending on the mode of operation. The flow path for an RBCC engine can be seen in Figure 3 .

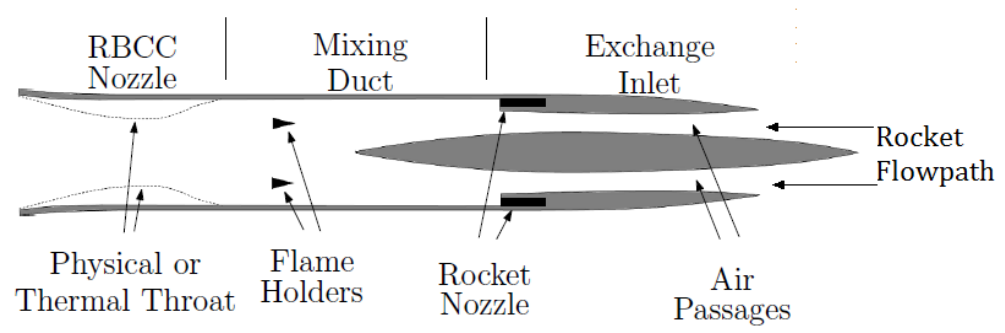

Figure 3: Rocket Flow path 10

The air enters through the air passages then begins mixing at the rocket nozzle in the mixing duct. The flame holders then ignite the flow and a throat exists downstream of the mixing duct to allow for further expansion of the flow if desired.

The rocket based combined cycle has 4 phases; ejector, ram jet, scram jet, and the 
pure rocket phase. Figure 4 a) shows the ejector mode, b) shows the ramjet mode and c) shows the rocket mode. These modes will be discussed in more detail following.

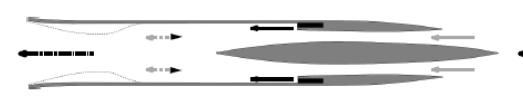

(a) Ejector Mode

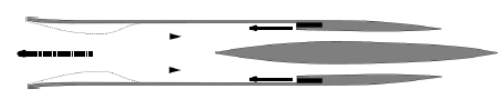

(c) Rocket Mode

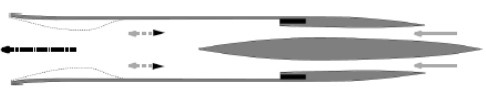

(b) Ramjet/Scramjet Mode

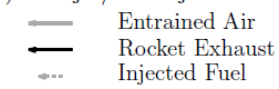

Injected Fuel

Figure 4: RBCC Engine modes 10

The ejector phase begins at low altitude and zero velocity and continues through the subsonic to sonic regime. In this phase, focus is on the entrainment process into the mixing duct as there is little air being driven into the vehicle by the vehicles speed initially. Entrainment is the process of drawing and accelerating atmospheric air in the free stream outside of the engine. The desired scenario is to create a choked entrained air flow with the duct to maximize the entrained mass flow. In Figure 4 a), the ejector is in operation and free stream flow is entrained into the duct with the flame holders, where it is ignited with the fuel for diffusion and after burning to increase thrust and expanded through the physical throat. This increases the efficiency of the engine over a pure rocket mode as can be seen in Figure 2.

After the vehicle reaches sonic flight conditions, the ram jet phase begins. The ram jet mode takes place from Mach 2 up until Mach 5-7. It is at the ram jet phase that compressed air would be used to ignite the fuel. While the operation of ram jet engines do function at lower Mach numbers, it is not until Mach 2 that high enough efficiencies are obtained [13]. A ramjet functions by allowing compressed air to enter into the engine and convert the inbound airflow from a high dynamic / low static pressure stream to a high static / low dynamic stream to allow for efficient combustion [13]. A ram jet can be as simple as a fuel injection section coupled with an entrained air section. 
After the ram jet phase is the scram jet phase which functions from approximately Mach 5-7 through to Mach 20 [14]. Scram jet technology is still under development with vehicles such as Boeings X-43, X-51, and various japanese studies investigating the potential for such a mode of operation [15, 16]. There are many difficulties in this stage such as the combustion of supersonic air flow. More work is required into scramjet technology to prove it's effectiveness. The scramjet phase is theorized to work up until $75 \mathrm{~km}$ altitude where the engine would convert to a pure rocket mode of operation.

The last engine mode is the pure rocket mode. Here there is no expectation of air from the atmosphere to be entering the engine to be utilized for thrust augmentation. The flow would be expanded out of the RBCC nozzle at the aft portion of the engine as is shown in Figure 4 c).

The focus of this work is on the ejector mode of operation.

\section{$1.3 \quad$ Ejectors}

Ejectors have many applications such as refrigeration cycles, chemical lasers and aerospace propulsion. They have been studied as early as 1942 by Keenan [17] where a simple 1 dimensional analysis is conducted assuming ideal gas and conservation of mass, momentum and energy. Ejectors have a number of primary variables of interest which are the entrainment ratio (the ratio of secondary to primary mass flow $(\omega)$ ), mixing length ratio (the length to diameter ratio (L/D) of the mixing duct), and pressure recovery (the ratio of initial total pressure to outlet total pressure).

Some of the first experimental analysis of the supersonic air-air ejector effect is analyzed by Fabri et al. [18, 19]. Fabri et al. show that for a given pressure ratio and nozzle, there are three types of flow through the secondary stream; A Fabri choke, in which the secondary flow is choked by the expanding plume of the primary flow 


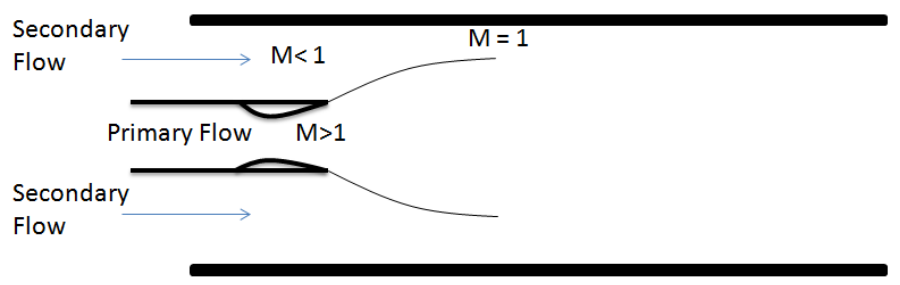

Figure 5: Fabri choke

shown in Figure 5, a saturated supersonic flow, in which the primary flow accelerates the secondary flow to sonic conditions and the duct acts as the aerodynamic choke shown in Figure 6, and a subsonic condition in which the secondary flow is not choked in any way despite there being a primary supersonic flow. An ideal circumstance is to choke the flow aerodynamically in a saturated supersonic flow. This means that the maximum mass flow of secondary flow is achieved due to choking at the minimum duct area as opposed to the Fabri choke where the minimum area would be reduced due to the difference between expanding plume and remaining area from the secondary stream duct.

Fabri [18] tests a cylindrical, axisymmetric flow and uses a quasi-one dimensional method to predict the flow structure of the primary stream. Using the conservation of mass, momentum, and energy from the inlet of both the secondary and primary flow, the outlet is solved for by summing the two flows and assuming uniform pressure between the flow and outlet conditions. Fabri's analysis also gives some of the flow patterns developed but does not give insight into the properties of the gas through the mixing chamber. Fabri's analysis also does not take into account viscous factors though a correction for wall friction is added.

Addy 20] then adds to Fabri's analysis using the same conditions of cylindrical, axisymmetric flow with a perfect gas by incorporating a method of characteristics on the primary plume. The method of characteristics allows for the shape of the 


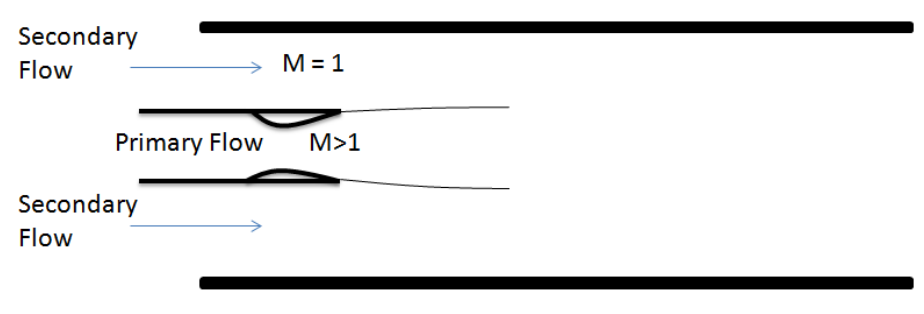

Figure 6: Saturated Supersonic Flow

expanding plume to be more fully incorporated into the control volume analysis. Addy also adds viscous effects into Fabri's initial analysis. The analysis provides for prediction of additional information in the mixing section of the ejector, as well as predicting the parameters at the inlet of the secondary stream. Addy also focuses on the supersonic-supersonic cases to further determine properties inside ejectors [20,21].

While both Fabri's and Addy's work on the prediction of characteristics within the mixing section gives some insight into the flow behavior, both assume a fully mixed outlet without regard to the length of the mixing section. Papamschou [22] analyses the flow under non ideal, partially mixed conditions. Both streams are investigated individually with the boundary calculated assuming pressure continuity. Empirical relations are then used for the shear stresses to describe the mixing layer. This method requires that both streams have the same initial pressure which limits the cases this method can be applied to.

There has been extensive extension by numerous authors of the quasi 1-D methods though most apply to circular and axisymmetric cases. Some examples of other quasi 1-D analysis are provided by Emanuel 23] who compares Fabri's analysis to other 1-D models, Huang [24] who validates against experiment a quasi 1-D approach to predicting entrainment performance when the primary flow is choked and the secondary flow reaches a critical choking condition, Han [25] investigates the effects of molecular weight with respect to the quasi-one dimensional model, and Del Valle [26] 
approaches Huang's analysis by generating the primary flow characteristics based on perturbation theory.

The quasi 1-D approach gives insight into flow characteristics but is often relegated to simple geometries and ideal conditions. In order to investigate realistic geometries or cases outside the realm of the quasi 1-D approach, experiments, visualization, and numerical analysis are conducted.

Using Computation Fluid Dynamics (CFD) to determine the behavior of an ejector requires accurate assessment of the modeling. Bartosiewicz [27 investigates different turbulence models for use in capturing the shock locations and strength in CFD of an ejector engine. Bartosiewicz investigates a number of different pressure ratios between the entrained air and nozzle flow in order to validate the use of CFD for ejectors. He is able to validate using CFD as a tool for ejector analysis and then compares 6 turbulence models to show that the $\mathrm{k}-\omega$ Menter SST model performs with the highest accuracy. CFD is an invaluable tool for predicting and optimizing ejector geometries and parameters prior to experimentation. CFD has been used to validated and predict a number of different geometric conditions for ejectors such as the placement of the nozzles in an ejector geometry by Zhu et al. and Varga et al. [28, 29]. Varga et al. also uses numerical analysis to validate the effect of the ratio of primary flow area to mixing area and primary flow throat area to mixing area [30. These numerical results were typically found to be within $10 \%$ of experimental results.

Numerical and experimental studies have been undertaken to determine the effect of the geometric ratios within gas ejectors. In a design trade off study by Jahingir and Huque [31, a combustion model is used to investigate the effects of the mixing length ratio $(\mathrm{L} / \mathrm{D})$ vs primary to secondary flow area vs inlet to expansion ratio. A sufficient L/D for a fully mixed flow has been cited by a number of authors as being between $5-10[32,33$ and this is more thoroughly examined by Jahingir and Huque. A 28 configuration study is run with constant total conditions at the secondary stream 
inlet and combusted rocket conditions from the primary stream. The ratio of secondary to primary flow changing shows different constriction ratios and its effects on the entrainment ratio $\left(\dot{m}_{\text {air }} / \dot{m}_{r}\right)$. It is found that the entrainment ratio generally increases as the secondary flow stream area increases. It is also found that the entrainment ratio increases as expansion ratio increases. Ejector compression ratio, or the ratio of exit total pressure to secondary total pressure at inlet decreases as the mixing ratio $(\mathrm{L} / \mathrm{D})$ and expansion ratio are increased while the ejector compression ratio increases as a result of decreasing the constriction ratio (smaller secondary area to primary area). It is found that the same result occurs in terms of ejector mixer thrust efficiency as ejector compression ratio, this makes sense as total pressure can be looked at as an energy density of the flow.

Lee et al. investigate the results of varied ejector throat area [34]. The throat of the ejector is kept constant while the nozzle throat is allowed to change. the pressure ratio is tested against various throat/ejector area ratios and at various pressures. It is shown that entrainment is a result of both pressure ratio of the two flows (where the primary motivating flow increases) and ratio between the ejector throat and the mixing duct throat.

The research group lead by Lineberry and Landrum investigate a Strujet engine configuration at great length in an effort to determine the effectiveness of changing the ejector configuration in a constant area entrained stream. The strutjet is pictured in Figure 7. Experimental and numerical investigation of a single nozzle in a strutjet configuration shows that a Fabri choke occurs rather than an aerodynamic choke 35 [36]. Further analysis into multiple rocket streams is conducted. Here the mass flow is kept constant with nozzles designed for one Mach number while ejector configuration is altered by changing the number of rockets from 1 to 2 [37] [38]. The research shows the effect of a single and dual nozzle configuration in terms of entrainment. It is found for their configurations that the entrainment ratio is almost identical when 


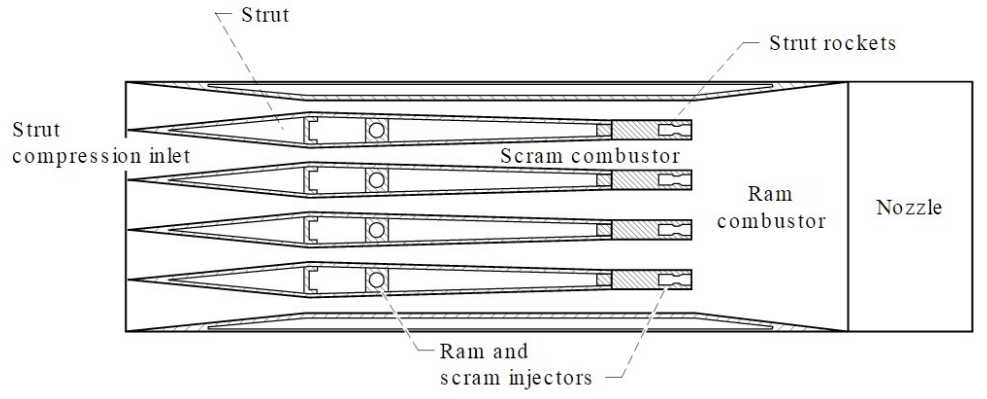

Figure 7: Features of a strutjet configuration in a ramjet engine [39]

matching the primary mass flows while increasing the number of rocket streams. As with the single nozzle configuration, Fabri choking occurs.

The geometry of the nozzle in an ejector can affect the entrainment ratios and mixing greatly. Mixing is assessed by the mixing length required. A variety of nozzle geometries are investigated such as a Clover shape by Samitha et al. [40]. Through experiment and numerical analysis Samitha et al. show that mixing length is reduced using this nozzle thereby having enhanced mixing. Rao and Jagadeesh show that a lobed nozzle similar to Samitha's clover nozzle also increases entrainment as much as $30 \%$ compared to a conical nozzle [41. Others have researched various concept designs such as the plug nozzles and dual bell nozzles which function for different ambient pressures, however not much consideration is given for entrainment as these are primarily researched for rocket driven vehicles [42]. The different nozzle designs attempt to increase shear layer area between the free stream and primary rocket stream to enhance mixing and entrainment. The research group at Carleton follows a similar strategy with the Exchange Inlet Nozzle.

\subsection{Exchange Inlet}

The Exchange Inlet is a nozzle design being researched at Carleton University. The goal of the Exchange Inlet is to increase mixing with a shorter mixing length and 
improve entrainment over other nozzle types at a given rocket pressure. The challenge for the ejector stage is to create a rocket stream that can transfer the high energy of the rocket exhaust into the free stream. The methodology of the Exchange Inlet is to increase the area of the shear layer between the two streams (free stream and rocket) such that the free stream reaches a choked level of operation at lower rocket pressures and rocket mass flows. The Exchange Inlet is pictured in Figure 12, In the Exchange Inlet, the rocket flow passes through the choke point of the nozzle (A), and then expands through the flow channel into several annular exhaust ports (B) and then out into the engine. When the expanded flow enters the engine, it mixes with the free stream, imparting some of the energy and accelerating the free stream air. The Exchange Inlet can be designed for any Mach number and mass flow [43].

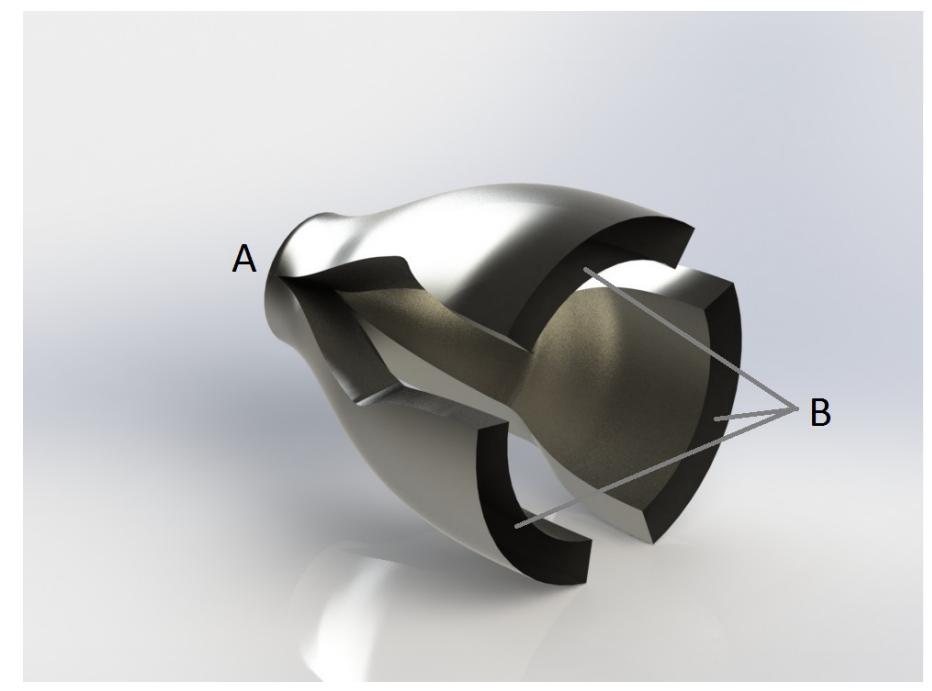

Figure 8: Exchange Inlet Nozzle

The Exchange Inlet is a design that can be changed depending on the needs of the rocket (Mach, Geometry, $U$ ). Free-stream air is entrained through the open areas of the nozzle and mixes with the annular flow coming out of the exhaust ports. An increase in the free-stream air $\left(\dot{m}_{\text {air }}\right)$ per rocket mass flow $\left(\dot{m}_{r}\right)$ increases the entrainment ratio which means more momentum is being transferred into the free stream by the rocket, which makes for an increased efficiency of the ejector. 


\subsection{Problem Statement}

At Carleton University, a nozzle design called the Exchange Inlet is being considered for use in a rocket based combined cycle engine. While research has been conducted into some of the losses present and some of the performance at various conditions, a comparison between the Exchange Inlet and other nozzle types has not been conducted in a realistic RBCC engine setting.

From previous work, it is predicted an annular nozzle entrains air better than a circular nozzle and the Exchange Inlet further enhances mixing and thereby has better entrainment properties for a rocket based combined cycle when compared to a circular nozzle $[44-46]$. At one of the more critical stages, the ejector stage, the Exchange Inlet is sought to improve performance over that of a circular nozzle.

Before a design is brought to reality, it is tested in a simulation realm. In this work, the validity of a computational code for an air breathing application based on completed experiments is conducted for both a circular nozzle design as well as the Exchange Inlet design. With close agreement between the available experimental data and the numerical data, further analysis into the rocket performance can be conducted. The analysis is extended to various operating rocket pressures and engine geometries to assess the differences in entrainment and mixing between the Exchange Inlet nozzle and circular nozzle. 


\section{Chapter 2}

\section{3-D Computational Theory}

Determining the key aspects of a flow inside a rocket engine is a complex issue. However, the use of computers allow for simulations to be done that give accurate accounts of what is occurring inside the region of interest as well as some detail into the potential flow interactions. Ejector flows incorporate large temperature and density gradients, compressibility effects, and shocks at various points. As such, the code utilized must be able to accurately capture these complex features without diverging. Also,a post processor must be used in order to analyze all these effects.

Computer technology still continues to follow the Moore's Law trend of doubling every 2 years and as such makes computational fluid dynamics increasingly appealing [47, 48]. With such advances in computer technology, many software packages have become available. There are many software packages that are able to simulate such flows such as the commercially available CFX-Fluent or CFD - ACE. There are also open source solutions such as Open-foam and the NASA OVERFLOW package. The availability of software to do simulations has increased over the years as computer technology continues to advance, however, selecting an adequate code or package is important. For this project, a software package designed by the Japanese Aerospace Exploration Agency (JAXA) called Kodera is used. 

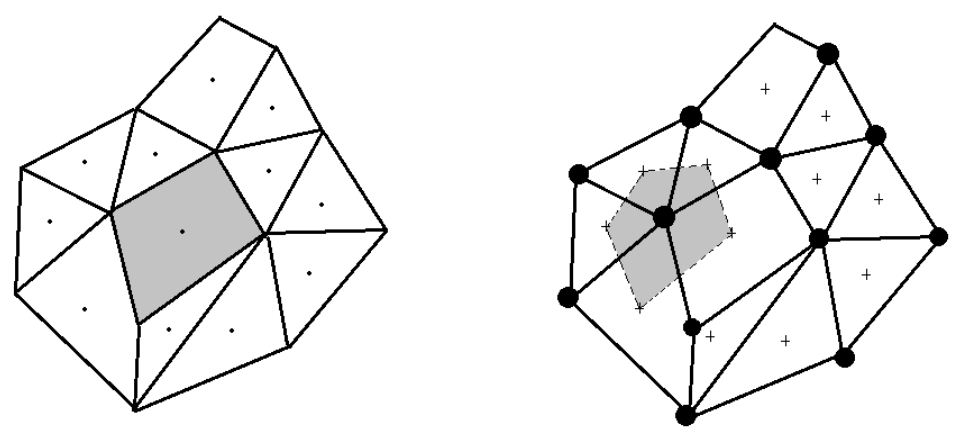

Figure 9: a) Cell Centered FVM b) Volume centered FVM

\subsection{Discretization of Domain}

In order to define the space and structures around and within which one wishes to simulate a flow, the domain must be discretized. In the finite volume method, the domain of interest is constructed using small structured or unstructured volumes that are the locations where calculations occur. The calculations can be done around a cell centered or volume centered method.

The cell centered method is pictured in Figure 9 a). In this method the nodes are placed in the center of the control volume while in b) the nodes are placed at the vertices of the volumes. In the volume centered FVM, a sub volume is created around the node by connecting the centers of the volumes with the midpoints of the edges. The Kodera software package uses the volume centered FVM.

For the discretization of the domain, there are similarly many packages available that allow the construction of structured and unstructured elements. Often these packages are coupled with a CFD package. The two ways a domain is constructed is with structured or unstructured elements 49 .

There are many types of structured grids such as cartesian, orthogonal and nonorthogonal grids. Structured grids can be more complex to generate in complex geometries, however are often sighted for their quick convergence and higher resolutions 
depending on the region of interest 49 .

In the structured method, since it is known how many nodes are in the different locations, the CFD method can be relatively straightforward, however in the unstructured method, since there can be multiple elements attached at each of the corners, a method must exist in the CFD code to calculate the contributions at each connected element.

Unstructured grids may use tetrahedron of various sizes to construct a grid around a complex geometry. The advantage this represents is that a grid can be quickly generated and can be generated for any geometry more easily. An unstructured grid also has an advantage for compressible flows where shock locations are not specifically

known beforehand. Due to both the complex geometry being simulated and the uncertainty of shock locations, an unstructured grid is used for this work.

At the wall there must be enough nodes to accurately calculate the viscous effects that may affect flow in the free stream. This means a biased accumulation of nodes must be created along no slip walls.

With a domain discretized, the solver can be used to solve the flow features of interest with the appropriate governing equations.

\subsection{Governing Equations}

The Navier-Stokes equations are a set of equations used to solve for the properties of a flow that has a greater amount of unknowns than there are equations.

The Navier-Stokes equations are given with three sets of equations

The momentum balance

$$
\frac{\partial}{\partial t}\left(\rho u_{i}\right)+\frac{\partial}{\partial x_{j}}\left(\rho u_{i} u_{j}\right)=-\frac{\partial}{\partial x_{j}}\left(p \delta_{i j}\right)+\frac{\partial \tau_{i j}}{\partial x_{j}}
$$

The conservation of mass or continuity 


$$
\frac{\partial \rho}{\partial t}+\frac{\partial}{\partial x_{j}}\left(\rho u_{j}\right)=0
$$

The conservation of energy

$$
\frac{\partial}{\partial t}(\rho E)+\frac{\partial}{\partial x_{j}}\left(\rho u_{j} H\right)=\frac{\partial}{\partial x_{j}}\left(u_{i} \tau_{i j}\right)-\frac{\partial q_{j}}{\partial x_{j}}
$$

where $\mathrm{H}$ is the total enthalpy per unit mass and $q_{j}$ is the heat flux vector, $E$ is the total energy per unit mass, and $\tau$ is the viscous stress tensor.

$$
\begin{gathered}
E=e+\frac{1}{2} u_{i} u_{i} \\
H=h+\frac{1}{2} u_{i} u_{i}=e+\frac{p}{\rho}+\frac{1}{2} u_{i} u_{i} \\
\tau_{i j}=2 \mu s_{i j}+\zeta s_{k k} \delta_{i j}
\end{gathered}
$$

And the species transport equation

$$
\frac{\partial}{\partial t}\left(\rho Y_{I}\right)+\frac{\partial}{\partial x_{j}}\left(\rho u_{j} Y_{I}\right)=\frac{\partial}{\partial x_{j}}\left(\rho D \frac{\partial Y_{I}}{\partial x_{j}}\right)+\dot{\omega}_{I}
$$

From above, $\rho$ is the density, $p$ is the pressure, $e$ is the energy, $h$ is the enthalpy, $x$ accounts for the location, $t$ is the time, $u$ are the velocity components, $\mu$ is the dynamic viscosity, $\zeta$ is the bulk viscosity, and the indices $i=1,2,3$ and $j$ is summed as $\sum_{j=1}^{3}$ for each i. $s_{i j}=\frac{1}{2}\left(\frac{\partial u_{i}}{\partial x_{j}}+\frac{\partial u_{j}}{\partial x_{i}}\right)$ is the strain rate tensor, $\zeta=-\frac{2}{3} \mu$ is found using Stokes Hypothesis, $\delta_{i j}$ is the Kronecker delta, and $k=1,2,3$ represent the index of their respective variables. $\mathrm{Y}$ is the partial density of each species I, D is the species diffusion coefficient and $\dot{\omega}_{I}$ is the source term assumed here to be zero. The viscous stress tensor can be simplified further with the stress deviator tensor $S_{i j}=s_{i j}-\frac{1}{3} s_{k k} \delta_{i j}$, so $\tau_{i j}$ becomes

$$
\tau_{i j}=2 \mu s_{i j}-\frac{2}{3} \mu s_{k k} \delta_{i j}=2 \mu S_{i j}
$$


In addition to the governing equations, density must be solved for as the compressibility effects cannot be neglected. In order to close the equations, additional equations are required as the total number of unknowns $\left(u_{i}, \rho, p, E, H, \mu\right.$ and $\left.q_{j}\right)$ is greater than the equations given. Using the equations of state and the relations between specific heats and energy we have the additional equations

$$
\begin{array}{lc}
p= & \rho R T \\
e= & c_{v} T \\
h= & e+\frac{p}{\rho}=c_{p} T
\end{array}
$$

Here $\mathrm{R}$ is the universal gas constant $8.314 \mathrm{~J} /(\mathrm{Kmol})$ and can be given specifically for each gas $R_{\text {specific }}=c_{p}-c_{v}$. The equation of state is used to supplement the energy equations and close the partial differential equations.

\subsection{Favre-Averaged Navier-Stokes}

The Favre-Averaged Navier-Stokes (FANS) equations are the density weighted average of the Navier-Stokes equations [50]. In the RANS flows, the properties of a given value are averaged in time and the Reynolds decomposition is used for each variable. Favre-Averaging incorporates a density weighted average to the Reynolds averaging. Using a generic flow variable q to represent either the temperature or velocity terms, the Reynolds average and Favre average of any variable are denoted by $\bar{q}$ and $\tilde{q}$ respectively. The weighted function of Favre averaging is given as :

$$
\tilde{q}=\frac{\overline{\rho q}}{\bar{\rho}}
$$

The value for any flow variable can be decomposed from its instantaneous value 
into it's mean (or average) and fluctuating components for both the Reynolds and Favre averages as

$$
\begin{aligned}
& q=\bar{q}+q^{\prime} \\
& q=\tilde{q}+q^{\prime \prime}
\end{aligned}
$$

where $q$ is the instantaneous value and the fluctuations are (') and (") for the Reynolds and Favre average respectively.

The operators used extensively in the FANS derivations are

$$
\begin{gathered}
\overline{q^{\prime}}=\overline{q-\bar{q}}=0 \\
\overline{\rho q^{\prime \prime}}=\bar{\rho} \tilde{q^{\prime \prime}}=0 \quad \overline{q^{\prime \prime}}=\overline{q-\tilde{q}}=\bar{q}-\tilde{q} \neq 0 \\
\widetilde{\frac{\partial u_{i}}{\partial x_{j}}}=\frac{\partial \tilde{u}_{i}}{\partial x_{j}}
\end{gathered}
$$

Using these relations, the Navier-Stokes equations can be re-written with the averaged terms as follows. Referring to Equation (1) of the Navier-Stokes Equation for momentum

$$
\frac{\partial}{\partial t}\left(\rho u_{i}\right)+\frac{\partial}{\partial x_{j}}\left(\rho u_{i} u_{j}\right)=-\frac{\partial}{\partial x_{j}}\left(p \delta_{i j}\right)+\frac{\partial \tau_{i j}}{\partial x_{j}}
$$

can be decomposed and averaged to

$$
\begin{gathered}
\overline{\left.\frac{\partial}{\partial t}\left(\rho\left(\tilde{u}_{i}\right)+u_{i}^{\prime \prime}\right)\right)+\frac{\partial}{\partial x_{j}}\left(\rho\left(\tilde{u}_{i}+u_{i}^{\prime \prime}\right)\left(\tilde{u}_{j}+u_{j}^{\prime \prime}\right)\right)}=-\overline{\frac{\partial}{\partial x_{j}}\left(p \delta_{i j}\right)+\frac{\partial \tau_{i j}}{\partial x_{j}}} \\
\frac{\partial}{\partial t}\left(\bar{\rho} \tilde{u}_{i}\right)+\frac{\partial}{\partial x_{j}}\left(\bar{\rho} \tilde{u}_{i} \tilde{u}_{j}+\overline{\left.\rho u_{i}^{\prime \prime} u_{j}^{\prime \prime}\right)}\right)=-\frac{\partial}{\partial x_{j}}\left(\bar{p} \delta_{i j}\right)+\frac{\partial \bar{\tau}_{i j}}{\partial x_{j}}
\end{gathered}
$$

and rearranging such that the term $\overline{\rho u_{i}^{\prime \prime} u_{j}^{\prime \prime}}$, often referred to as the Reynolds stress, is on the right hand side. This produces the FANS equation for momentum.

$$
\frac{\partial}{\partial t}\left(\bar{\rho} \tilde{u}_{i}\right)+\frac{\partial}{\partial x_{j}}\left(\bar{\rho} \tilde{u}_{i} \tilde{u}_{j}\right)=-\frac{\partial}{\partial x_{j}}\left(\bar{p} \delta_{i j}\right)+\frac{\partial}{\partial x_{j}}\left(\bar{\tau}_{i j}-\overline{\rho u_{i}^{\prime \prime} u_{j}^{\prime \prime}}\right)
$$


Applying the same operations to the conservation of mass (2)

$$
\frac{\partial \rho}{\partial t}+\frac{\partial}{\partial x_{j}}\left(\rho u_{j}\right)=0
$$

$$
\overline{\frac{\partial \rho}{\partial t}+\frac{\partial}{\partial x_{j}}\left(\rho \tilde{u}_{j}+\rho u_{j}^{\prime \prime}\right)}=0
$$

$$
\begin{gathered}
\overline{\frac{\partial \rho}{\partial t}+\frac{\partial}{\partial x_{j}}\left(\rho \tilde{u}_{j}+\rho u_{j}^{\prime \prime}\right)}=\frac{\partial \bar{\rho}}{\partial t}+\frac{\partial}{\partial x_{j}}\left(\bar{\rho} \tilde{u}_{j}\right)+\frac{\partial}{\partial x_{j}}\left(\overline{\rho u_{j}^{\prime \prime}}\right)=\frac{\partial \bar{\rho}}{\partial t}+\frac{\partial}{\partial x_{j}}\left(\bar{\rho} \tilde{u}_{j}\right) \\
\frac{\partial \bar{\rho}}{\partial t}+\frac{\partial}{x_{j}}\left(\bar{\rho} \tilde{u}_{j}\right)=0
\end{gathered}
$$

Equation (11) is the FANS equation for conservation of mass. From the energy equation (3), it is useful to identify the individual components since they are much more involved in their decompositions. It is also worthwhile to define the Turbulent Kinetic Energy (TKE) $k=\sum_{i=1}^{3} \frac{1}{2} \widetilde{u_{i}^{\prime \prime} u_{i}^{\prime \prime}}$.

$$
\underbrace{\frac{\partial}{\partial t}(\rho E)}_{I}+\underbrace{\frac{\partial}{\partial x_{j}}\left(\rho u_{j} H\right)}_{I I}=\underbrace{\frac{\partial}{\partial x_{j}}\left(u_{i} \tau_{i j}\right)}_{I I I}-\underbrace{\frac{\partial q_{j}}{\partial x_{j}}}_{I V}
$$


I)

$$
\begin{aligned}
\overline{\frac{\partial}{\partial t}(\rho E)} & =\overline{\frac{\partial}{\partial t}\left[\rho\left(\tilde{e}+e^{\prime \prime}+\sum_{i=1}^{3} \frac{1}{2}\left(\tilde{u}_{i}+u_{i}^{\prime \prime}\right)\left(\tilde{u}_{i}+u_{i}^{\prime \prime}\right)\right)\right]} \\
& =\frac{\partial}{\partial t}\left[\bar{\rho}\left(\tilde{e}+\sum_{i=1}^{3} \frac{1}{2} \tilde{u}_{i} \tilde{u}_{i}\right)+\sum_{i=1}^{3} \bar{\rho} \frac{1}{2} \widetilde{\left.u_{i}^{\prime \prime} u_{i}^{\prime \prime}\right]}\right. \\
& =\frac{\partial}{\partial t}\left[\bar{\rho}\left(\tilde{e}+\sum_{i=1}^{3} \frac{1}{2} \tilde{u}_{i} \tilde{u}_{i}+k\right)\right] \\
& =\frac{\partial}{\partial t}(\bar{\rho} \tilde{E})
\end{aligned}
$$

II)

$$
\begin{aligned}
\overline{\frac{\partial}{\partial x_{j}}\left(\rho u_{j} H\right)} & =\overline{\frac{\partial}{\partial x_{j}}\left[\rho\left(\tilde{u}_{j}+u_{j}^{\prime \prime}\right)\left(\tilde{h}+h^{\prime \prime}+\sum_{i=1}^{3} \frac{1}{2}\left(\tilde{u}_{i}+u_{i}^{\prime \prime}\right)\left(\tilde{u}_{i}+u_{i}^{\prime \prime}\right)\right)\right]} \\
& =\frac{\partial}{\partial x_{j}}\left[\rho\left(\tilde{u}_{j}+u_{j}^{\prime \prime}\right)\left(\tilde{h}+h^{\prime \prime}+\sum_{i=1}^{3}\left(\frac{1}{2} \tilde{u}_{i} \tilde{u}_{i}+u_{i}^{\prime \prime} \tilde{u}_{i}+\frac{1}{2} u_{i}^{\prime \prime} u_{i}^{\prime \prime}\right)\right)\right] \\
& =\frac{\partial}{\partial x_{j}}\left(\bar{\rho} \tilde{u}_{j} \tilde{h}+\bar{\rho} \tilde{u}_{j} \frac{1}{2} \tilde{u}_{i} \tilde{u}_{i}+\bar{\rho} \tilde{u}_{j} k+\overline{\rho u_{j}^{\prime \prime} h^{\prime \prime}}+\overline{\rho u_{j}^{\prime \prime} u_{i}^{\prime \prime}} \tilde{u}_{i}+\frac{1}{2} \overline{\left.\rho u_{j}^{\prime \prime} u_{i}^{\prime \prime} u_{i}^{\prime \prime}\right)}\right. \\
& =\frac{\partial}{\partial x_{j}}\left(\bar{\rho} \tilde{u}_{j} \tilde{H}+\overline{\rho u_{j}^{\prime \prime} h^{\prime \prime}}+\overline{\rho u_{j}^{\prime \prime} u_{i}^{\prime \prime}} \tilde{u}_{i}+\frac{1}{2} \overline{\rho u_{j}^{\prime \prime} u_{i}^{\prime \prime} u_{i}^{\prime \prime}}\right)
\end{aligned}
$$

III)

$$
\begin{aligned}
\overline{\frac{\partial}{\partial x_{j}}\left(u_{i} \tau_{i j}\right)} & =\overline{\frac{\partial}{\partial x_{j}}\left(\left(\tilde{u}_{i}+u_{i}^{\prime \prime}\right) \tau_{i j}\right)} \\
& =\frac{\partial}{\partial x_{j}}\left(\tilde{u}_{i} \bar{\tau}_{i j}+\overline{u_{i}^{\prime \prime} \tau_{i j}}\right)
\end{aligned}
$$

IV)

$$
\frac{\overline{\partial q_{j}}}{\partial x_{j}}=\frac{\partial \bar{q}_{j}}{\partial x_{j}}
$$

Gathering all the terms and combining back into one equation yields the FANS equations for energy. 


$$
\frac{\partial}{\partial t}(\bar{\rho} \tilde{E})+\frac{\partial}{\partial x_{j}}\left(\bar{\rho} \tilde{u}_{j} \tilde{H}\right)=\frac{\partial}{\partial x_{j}}\left(\left(\bar{\tau}_{i j}-\overline{\rho u_{i}^{\prime \prime} u_{j}^{\prime \prime}}\right) \tilde{u}_{i}\right)-\frac{\partial}{\partial x_{j}}\left(\bar{q}_{j}+\overline{\rho u_{j}^{\prime \prime} h^{\prime \prime}}\right)+\frac{\partial}{\partial x_{j}}\left(-\frac{1}{2} \overline{\rho u_{j}^{\prime \prime} u_{i}^{\prime \prime} u_{i}^{\prime \prime}}+\overline{u_{i}^{\prime \prime} \tau_{i j}}\right)
$$

Using the same rules, the FANS equation for the species transport is given as

$$
\begin{gathered}
\overline{\left.\frac{\partial}{\partial t}\left(\rho \tilde{Y}_{I}+Y_{I}^{\prime \prime}\right)\right)+\frac{\partial}{\partial x_{j}}\left(\rho\left(\tilde{Y}_{I}+Y_{I}^{\prime \prime}\right)\left(\tilde{u}_{j}+u_{j}^{\prime \prime}\right)\right)}=\overline{\frac{\partial}{\partial x_{j}}\left(\rho D_{I} \frac{\partial}{\partial x_{j}}\left(\tilde{Y}_{I}+Y_{I}^{\prime \prime}\right)\right)} \\
\frac{\partial}{\partial t}\left(\bar{\rho} \tilde{Y}_{I}\right)+\frac{\partial}{\partial x_{j}}\left(\bar{\rho} \tilde{Y}_{i} \tilde{u}_{j}+\overline{\rho Y_{I}^{\prime \prime} u_{j}^{\prime \prime}}\right)=\frac{\partial}{\partial x_{j}}\left(\bar{\rho} D_{I} \frac{\partial \tilde{Y}_{I}}{\partial x_{j}}\right)+\frac{\partial}{\partial x_{j}}\left(\bar{\rho} D_{I} \frac{\overline{\partial Y_{I}^{\prime \prime}}}{\partial x_{j}}\right) \\
\frac{\partial}{\partial t}\left(\bar{\rho}_{I}\right)+\frac{\partial}{\partial x_{j}}\left(\bar{\rho} \tilde{u}_{j} \tilde{Y}_{I}\right)=\frac{\partial}{\partial x_{j}}\left(\bar{\rho} D_{I} \frac{\partial \tilde{Y}_{I}}{\partial x_{j}}-\overline{\rho Y_{I}^{\prime \prime} u_{j}^{\prime \prime}}\right)+\left(\bar{\rho} D_{I} \frac{\overline{\partial Y_{I}^{\prime \prime}}}{\partial x_{j}}\right)
\end{gathered}
$$

Equations (10) (11), (12), (13) make up the FANS equations and we notice some extra terms that are not distinctly evident in the N-S equations (1), (2) (3), (4). The terms are a result of the turbulence and are $-\overline{\rho u_{i}^{\prime \prime} u_{j}^{\prime \prime}}, \overline{\rho u_{j}^{\prime \prime} h^{\prime \prime}},-\frac{1}{2} \overline{\rho u_{j}^{\prime \prime} u_{i}^{\prime \prime} u_{i}^{\prime \prime}}$, and $\overline{u_{i}^{\prime \prime} \tau_{i j}}$ which are the Reynolds stress, the Reynolds heat flux, the transport term for turbulent kinetic energy by turbulent velocity fluctuations, and the work done by viscous stress due to turbulent velocity fluctuations respectively.

Equation (5) for the viscous shear tensor must also be Favre-Averaged. A $3^{\text {rd }}$ order polynomial is used to determine the dynamic viscosity. The equation for the dynamic viscosity takes the form of

$$
\begin{gathered}
\mu(I)=\left(C \mu_{I 1} * T+C \mu_{I 2} * T^{2}+C \mu_{I 3} * T^{3}+C \mu_{I 4}\right. \\
\mu=\sum_{I=1}^{I \max } \mu(I) * M_{I}
\end{gathered}
$$

where $\mathrm{I}$ is the indice for the species and $C \mu$ are the constants for a given species. It is also useful to work in terms of kinematic viscosity instead of dynamic viscosity where $\nu=\mu / \rho$. Applying both of these operations to equation (5) yields 


$$
\begin{gathered}
\overline{\tau_{i j}=2\left(\rho\left(\tilde{\nu}+\nu^{\prime \prime}\right)\left(\tilde{S}_{i j}+S_{i j}^{\prime \prime}\right)\right.} \\
\bar{\tau}_{i j}=2 \bar{\rho} \tilde{\nu} \tilde{S}_{i j}+2 \overline{\rho \nu^{\prime \prime} S_{i j}^{\prime \prime}}
\end{gathered}
$$

where it is assumed that $2 \bar{\rho} \tilde{\nu} \tilde{S}_{i j}$ is $>>2 \overline{\rho \nu^{\prime \prime} S_{i j}^{\prime \prime}}$ so the final equation for the stress term is

$$
\bar{\tau}_{i j}=2 \bar{\rho} \tilde{\nu} \tilde{S}_{i j}
$$

The equations of state (6) to be used for closure, with Favre-Averaging is given as

$$
\bar{p}=\bar{\rho} R \tilde{T}
$$

Applying the same Favre-Averaging to Equations (7) and 8 gives

$$
\begin{gathered}
\tilde{e}=c_{v} \tilde{T} \\
\tilde{h}=\tilde{e}+\frac{\tilde{p}}{\bar{\rho}}=c_{p} \tilde{T}
\end{gathered}
$$

Where the specific heats are given for each species from a $5^{\text {th }}$ order polynomial of the form

$$
\begin{gathered}
c_{p}(I)=\left(C P_{I 1} * T+C P_{I 2} * T^{2}+C P_{I 3} * T^{3}+C P_{I 4} * T^{4}+C P_{I 5} * T^{5}+C P_{I 6}\right) * R \\
c_{p}=\sum_{I=1}^{I m a x} c_{p}(I) * M_{I}
\end{gathered}
$$

Where $\mathrm{I}$ is an indice representing a species, $\mathrm{CP}$ are the constants and $\mathrm{M}$ is the molar mass for a given species.

In dealing with the heat flux terms present in Equation $(12), q_{j}$, we apply Fouriers theorem which yields

$$
q_{j}=-\kappa \frac{\partial T}{\partial x_{j}}=-\frac{\kappa}{c_{p}} \frac{\partial h}{\partial x_{j}}=-\frac{\mu}{\operatorname{Pr}} \frac{\partial h}{\partial x_{j}}=-\frac{\rho \nu}{\operatorname{Pr}} \frac{\partial h}{\partial x_{j}}
$$


where $\kappa$ is the thermal conductivity and $\operatorname{Pr}=c_{p} \mu / \kappa$ is the Prandtl number. From here, applying the same averaging functions and following the same steps as with the FANS equations

$$
\begin{aligned}
\bar{q}_{j} & =-\frac{1}{\operatorname{Pr}} \overline{\rho\left(\tilde{\nu}+\nu^{\prime \prime}\right) \frac{\partial}{\partial x_{j}}\left(\tilde{h}+h^{\prime \prime}\right)} \\
& =-\frac{1}{\operatorname{Pr}}\left(\bar{\rho} \tilde{\nu} \frac{\partial \tilde{h}}{\partial x_{j}}+\overline{\rho \nu^{\prime \prime} \frac{\partial h^{\prime \prime}}{\partial x_{j}}}\right)
\end{aligned}
$$

Assuming $\bar{\rho} \tilde{\nu} \frac{\partial h}{\partial x_{j}}>>\overline{\rho \nu^{\prime \prime} \frac{\partial h^{\prime \prime}}{\partial x_{j}}}$ gives a final equation for the heat flux

$$
\bar{q}_{j}=-\frac{\bar{\rho} \tilde{\nu}}{\operatorname{Pr}} \frac{\partial \tilde{h}}{\partial x_{j}}
$$

A summary of the FANS Equations are

$$
\begin{gathered}
\frac{\partial}{\partial t}\left(\bar{\rho} \tilde{u}_{i}\right)+\frac{\partial}{\partial x_{j}}\left(\bar{\rho} \tilde{u}_{i} \tilde{u}_{j}+\overline{\rho u_{i}^{\prime \prime} u_{j}^{\prime \prime}}\right)=-\frac{\partial \bar{p} \delta_{i} j}{\partial x_{j}}+\frac{\partial}{\partial x_{j}}\left(2 \bar{\rho} \tilde{v} \tilde{S}_{i j}-\overline{\rho u_{i}^{\prime \prime} u_{j}^{\prime \prime}}\right) \\
\frac{\partial \bar{\rho}}{\partial t}+\frac{\partial}{x_{i}}\left(\bar{\rho} \tilde{u}_{j}\right)=0 \\
\frac{\partial}{\partial t}(\bar{\rho} \tilde{E})+\frac{\partial}{\partial x_{j}}\left(\bar{\rho} \tilde{u}_{j} \tilde{H}\right)=\frac{\partial}{\partial x_{j}}\left(\left(2 \bar{\rho} \tilde{v} \tilde{S}_{i j}-\overline{\left.\rho u_{j}^{\prime \prime} u_{i}^{\prime \prime}\right)} \tilde{u}_{i}\right)\right. \\
-\frac{\partial}{\partial x_{j}}\left(-\frac{\bar{\rho} \tilde{\nu}}{P r} \frac{\partial \tilde{h}}{\partial x_{j}}+\overline{\rho u_{j}^{\prime \prime} h^{\prime \prime}}\right)+\frac{\partial}{\partial x_{j}}\left(-\frac{1}{2} \overline{\rho u_{j}^{\prime \prime} u_{i}^{\prime \prime} u_{i}^{\prime \prime}}+\overline{u_{i}^{\prime \prime} \tau_{i j}}\right)
\end{gathered}
$$

The remaining unknowns are $-\overline{\rho u_{i}^{\prime \prime} u_{j}^{\prime \prime}}, \overline{\rho u_{j}^{\prime \prime} h^{\prime \prime}},-\frac{1}{2} \overline{\rho u_{j}^{\prime \prime} u_{i}^{\prime \prime} u_{i}^{\prime \prime}}, \overline{u_{i}^{\prime \prime} \tau_{i j}}$, and the TKE $k$. These remaining terms are handled using a turbulence model. 


\subsection{Turbulence Model}

There are many turbulence models that exist to close the equations due to the stress terms created by the fluctuating velocities in the FANS equations. The $\mathrm{k}-\omega$ model is a two equation model for the eddy viscosity that is used in this work. The remaining terms are modeled by relating them to the mean flow through scalar functions. The Reynolds Stress $-\overline{\rho u_{i}^{\prime \prime} u_{j}^{\prime \prime}}$ is modeled using

$$
-\overline{\rho u_{i}^{\prime \prime} u_{j}^{\prime \prime}}=2 \mu_{t} \overline{S_{i j}}-\frac{2}{3} \bar{\rho} k \delta_{i j}
$$

The Reynolds heat flux $\overline{\rho u_{j}^{\prime \prime} h^{\prime \prime}}$ is modeled based on an eddy turbulent thermal conductivity.

$$
\overline{\rho u_{j}^{\prime \prime} h^{\prime \prime}}=-\kappa_{t} \frac{\partial \tilde{T}}{\partial x_{j}}
$$

where $\kappa_{t}$ is the eddy turbulent thermal conductivity which can be related to the eddy turbulent viscosity and turbulent Prandtl number. This gives $\kappa_{t}=c_{p} \mu_{t} / P r_{t}$. Using this relation gives a the Reynolds Heat flux its final form

$$
\overline{\rho u_{j}^{\prime \prime} h^{\prime \prime}}=-\kappa_{t} \frac{\partial \tilde{T}}{\partial x_{j}}=\frac{c_{p} \mu_{t}}{P r_{t}} \frac{\partial \tilde{T}}{\partial x_{j}}
$$

Lastly, the TKE transport term and turbulent work term $-\frac{1}{2} \overline{\rho u_{j}^{\prime \prime} u_{i}^{\prime \prime} u_{i}^{\prime \prime}}$ and $\overline{u_{i}^{\prime \prime} \tau_{i j}}$ are modeled using the gradient diffusion hypothesis defined by

$$
-\frac{1}{2} \overline{\rho u_{j}^{\prime \prime} u_{i}^{\prime \prime} u_{i}^{\prime \prime}}+\overline{u_{i}^{\prime \prime} \tau_{i j}}=\left(\bar{\mu}+\sigma_{k} \mu_{t}\right) \frac{\partial k}{\partial x_{j}}
$$

where $\sigma_{k}$ is a calibration variable given by the turbulence model. This leaves the only unknowns as the turbulent eddy viscosity and TKE $\left(\mu_{t}\right.$ and $\left.k\right)$. 
The TKE equation is derived by multiplying the instantaneous momentum Equation (1) by the fluctuating velocity term $u_{i}^{\prime \prime}$.

$$
u_{i}^{\prime \prime}\left(\frac{\partial}{\partial t}\left(\rho u_{i}\right)+\frac{\partial}{\partial x_{j}}\left(\rho u_{i} u_{j}\right)\right)=u_{i}^{\prime \prime}\left(-\frac{\partial p \delta_{i} j}{\partial x_{j}}+\frac{\partial \tau_{i j}}{\partial x_{j}}\right)
$$

The TKE equation is then Reynolds averaged and through a similar derivation as the FANS equations the turbulent kinetic energy equation for FANS is

$$
\begin{aligned}
& \frac{\partial}{\partial t}(\bar{\rho} k)+\frac{\partial}{\partial x_{j}}\left(\bar{\rho} \tilde{u}_{j} k\right)=-\overline{\rho u_{i}^{\prime \prime} u_{j}^{\prime \prime}} \frac{\tilde{u}_{i}}{\partial x_{j}}-\overline{\tau_{i j} \frac{\partial u_{i}^{\prime \prime}}{\partial x_{j}}}+\frac{\partial}{\partial x_{j}}\left(-\frac{1}{2} \overline{\rho u_{j}^{\prime \prime} u_{i}^{\prime \prime} u_{i}^{\prime \prime}}+\overline{\tau_{i j} u_{i}^{\prime \prime}}\right) \\
& -\overline{u_{i}^{\prime \prime}} \frac{\partial \bar{p} \delta_{i} j}{\partial x_{i}}-\frac{\partial}{\partial x_{i}}\left(\bar{u}_{i}^{\prime \prime} p^{\prime}\right)+\overline{p^{\prime} \frac{\partial u_{i}^{\prime \prime}}{\partial x_{i}}}
\end{aligned}
$$

The terms $-\overline{\rho u_{i}^{\prime \prime} u_{j}^{\prime \prime}},-\frac{1}{2} \overline{\rho u_{j}^{\prime \prime} u_{i}^{\prime \prime} u_{i}^{\prime \prime}}$, and $\overline{u_{i}^{\prime \prime} \tau_{i j}}$ are again present and modeled with the assumptions from Equations 19 and 20 . This leaves the unknown terms $\overline{\tau_{i j} \frac{\partial u_{i}^{\prime \prime}}{\partial x_{j}}}$ which is the dissipation rate, and also the pressure terms. The final closure for the $k-\omega$ model assumes that the pressure terms are negligible and that the dissipation rate can be related to turbulent frequency $\omega$. In the $k-\epsilon$ model, the dissipation rate is modeled as

$$
\bar{\rho} \epsilon=\overline{\tau_{i j} \frac{\partial u_{i}^{\prime \prime}}{\partial x_{j}}}
$$

but the $\mathrm{k}-\omega$ model assumes that $\omega \propto \epsilon / k$. So

$$
\bar{\rho} \epsilon=\overline{\tau_{i j} \frac{\partial u_{i}^{\prime \prime}}{\partial x_{j}}}=\beta^{*} \bar{\rho} \omega k
$$

The k- $\omega$ Menter SST turbulence set of equations allow for a set of equations that deal well with the near wall field and far field (free stream). The k-epsilon model has been noted for it's great accuracy in the far field, while Wilcox' k- $\omega$ model is superior 
in the near wall region. Menter changes the epsilon equation into a $\omega$ equation by substituting $\epsilon=k \omega$ and using a blending function for the transition between the near wall field and far field. The model is governed by the following sets of equations 51 kinematic eddy viscosity:

$$
\mu_{T}=\frac{a_{1} \bar{\rho} k}{\max \left(a_{1} \omega, \Omega F_{2}\right)}
$$

Turbulence kinetic Energy:

$$
\frac{\partial}{\partial t}(\bar{\rho} k)+\frac{\partial}{\partial x_{j}}\left(\bar{\rho} \tilde{u}_{j} k\right)=\left(2 \mu_{t} \bar{S}_{i j}-\frac{2}{3} \bar{\rho} k \delta_{i j}\right) \frac{\partial \tilde{u}_{i}}{\partial x_{j}}-\beta^{*} \bar{\rho} k \omega+\frac{\partial}{\partial x_{j}}\left(\left(\bar{\mu}+\sigma_{k} \mu_{t}\right) \frac{\partial k}{\partial x_{j}}\right)
$$

Specific Dissipation Rate:

$$
\begin{aligned}
\frac{\partial}{\partial t}(\bar{\rho} \omega)+\frac{\partial}{\partial x_{j}}\left(\bar{\rho} \tilde{u}_{j} \omega\right)=\frac{\alpha}{\nu_{t}}( & \left.2 \mu_{t} \bar{S}_{i j}-\frac{2}{3} \bar{\rho} k \delta_{i j}\right) \frac{\partial \tilde{u}_{i}}{\partial x_{j}}-\beta^{*} \bar{\rho} \omega^{2} \\
& +\frac{\partial}{\partial x_{j}}\left(\left(\bar{\mu}+\sigma_{\omega} \mu_{t}\right) \frac{\partial k}{\partial x_{j}}\right)+2\left(1-F_{1}\right) \bar{\rho} \sigma_{\omega 2} \frac{1}{\omega} \frac{\partial k}{\partial x_{j}} \frac{\partial \omega}{\partial x_{j}}
\end{aligned}
$$

with the following additional equations for the remaining terms

$$
\begin{gathered}
\sigma_{k}=F_{1} \sigma_{k 1}+\left(1-F_{1}\right) \sigma_{k 2} \quad \sigma_{\omega}=F_{1} \sigma_{\omega 1} \quad \beta=F_{1} \beta_{1}+\left(1-F_{1}\right) \beta_{2} \\
\alpha=F_{1} \alpha_{1}+\left(1-F_{1}\right) \alpha_{2} \quad \alpha_{1}=\frac{\beta_{1}}{\beta^{*}}-\sigma_{\omega_{1}} \frac{\Upsilon^{2}}{\sqrt{\beta^{*}}} \quad \alpha_{2}=\frac{\beta_{2}}{\beta^{*}}-\sigma_{\omega_{2}} \frac{\Upsilon^{2}}{\sqrt{\beta^{*}}} \\
F_{1}=\tanh \left(\arg _{1}^{4}\right) \quad \arg _{1}=\min \left(\max \left(\frac{\sqrt{k}}{0.09 \omega d} \frac{500 \bar{\nu}}{d^{2} \omega}\right), \frac{4 \bar{\rho} \sigma_{\omega 2} k}{C D_{k \omega} d^{2}}\right) \\
F_{2}=\tanh \left(\arg _{2}^{2}\right) \quad \arg g_{2}=\max \left(\frac{2 \sqrt{k}}{0.09 \omega d}, \frac{500 \bar{\nu}}{d^{2} \omega}\right) \\
C D_{k \omega}=\max \left(2 \bar{\rho} \sigma_{\omega 2} \frac{1}{\omega} \frac{\partial k}{\partial x_{j}} \frac{\partial \omega}{\partial x_{j}}, 0\right) \quad \Omega=\sqrt{2 \tilde{\Omega}_{i j} \tilde{\Omega}_{i j}}
\end{gathered}
$$


with $\Omega$ as the variable for vorticity and the remaining constants are

$$
\begin{gathered}
\sigma_{k 1}=0.85 \quad \sigma_{\omega 1}=0.65 \quad \sigma_{k 2}=1.00 \quad \sigma_{\omega 2}=0.856 \\
\beta_{1}=0.075 \quad \beta_{2}=0.828 \quad \beta^{*}=0.09 \quad a_{1}=0.31 \quad \Upsilon=0.41
\end{gathered}
$$

\section{$2.5 \quad$ Kodera}

The software package used for this work is named Kodera and is used primarily by the Japanese Space Agency for much of its CFD research. It has been used in the past to perform many high speed flow simulations such as a transonic flow around an airplane and wing, a numerical study of a strut jet engine, and a high speed flow through a scramjet inlet at high altitude conditions [52 [54]. It uses a Lower Upper Symmetric Gauss Seidel Implicit method (LUSGS) with a local Courant Freidrichs condition (CFL) and the Harten, Lax, Van Leer, Einfeldt, Wada Riemann solver (HLLEW). The HLLEW Reimann solver couples a Monotonic Upstream Centered Scheme for Conservation Laws (MUSCL) with the Advection Upstream Splitting Method (AUSMDV) for the flux terms. The MUSCL scheme allows for a second order spatially accurate solver that is able to accurately capture shock discontinuities [55, 56]. The Venkatakrishnan Limiter is used to increase the rate of convergence. The turbulence model used is the Menter $k-\omega$ shear stress transport model. 


\section{Chapter 3}

\section{Methodology}

\section{$3.1 \quad$ Geometry}

Two geometries are simulated in this thesis. Both reflect engine geometry used by JAXA. The two geometries are designed such that the mixing length ratio (L/D) are equal, the rocket stagnation conditions are equal $\left(\rho_{0}, T_{o}, p_{o}\right)$, and the ratio of entrained air flow to total flow area are equal in both geometries. In both geometries two rocket nozzles are used. Both nozzles are designed for Mach 2.0 when including viscous effects and placed $140 \mathrm{~mm}$ downstream of the engine inlet. The difference between the two geometries are that the nozzle designs are different. For the Twin Circular configuration, two simple conical expansions are used while the Exchange Inlet design uses two Exchange Inlet nozzles currently under research at Carleton University.

\subsubsection{Twin Circular Configuration}

The twin circular nozzles are converging-diverging rocket nozzles. Typical circular nozzles are bell nozzles, De Laval nozzles, and simple conical nozzles. Simple conical nozzles are used in this work. There are two nozzles with symmetry along the center plane between them. Figure 10 shows half engine of the Twin Circular configuration 
with the flow direction. The hatched darker surface represents the Symmetry plane. All measurements are in millimeters $[\mathrm{mm}]$ and the $18 \mathrm{~mm}$ diameter circle represents the exit of the nozzle.

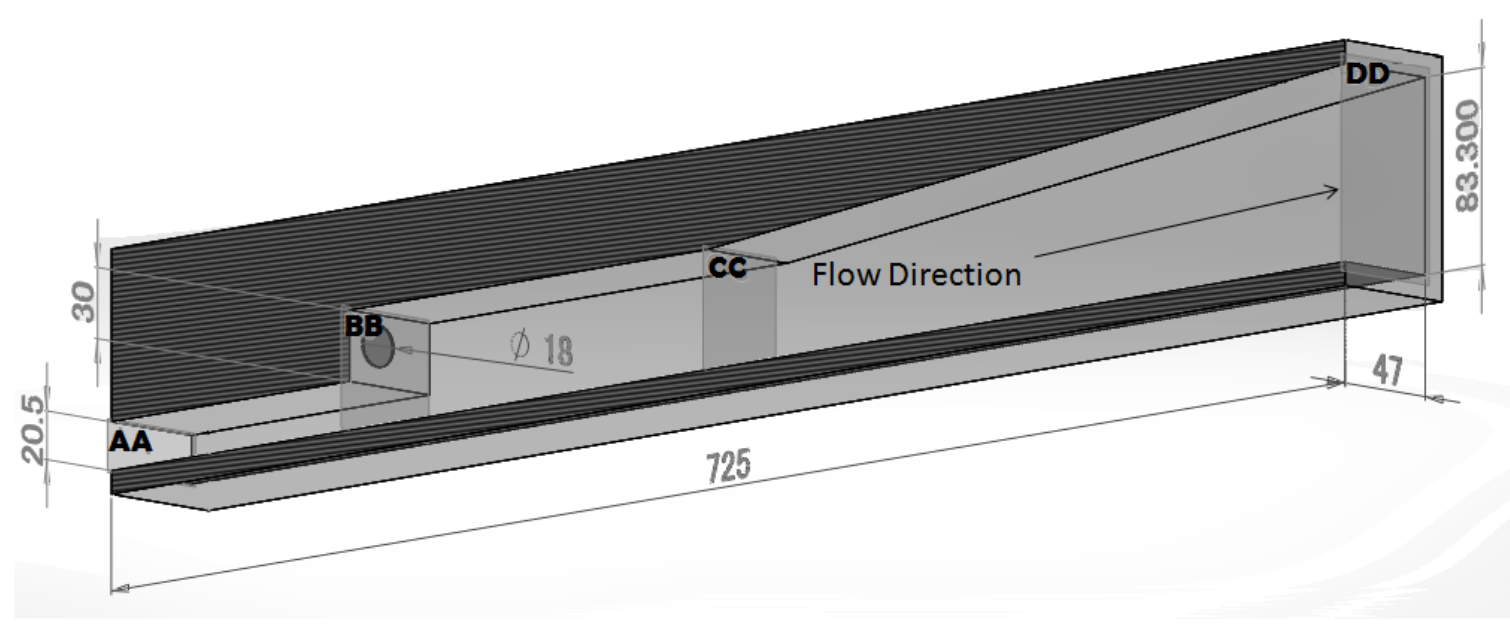

Figure 10: Twin Circular configuration 3D view

It is useful to describe the geometry in terms of areas and planes. In Figure 10 AA represents the entrainment duct inlet, BB represents the Rocket Nozzle Exit Plane (RNEP), CC is the beginning of the geometric expansion and is called the Expansion Section Plane (ESP), and DD is the Engine Exit Plane (EEP). The space between $\mathrm{AA}$ and $\mathrm{BB}$ is the entrainment duct, between $\mathrm{BB}$ and $\mathrm{CC}$ is the straight duct, and between $\mathrm{CC}$ and $\mathrm{DD}$ is the expanding duct. The entire engine length is $725 \mathrm{~mm}$ and the length from AA to $\mathrm{CC}$ is $350 \mathrm{~mm}$ and length of the expanding duct is 375 $\mathrm{mm}$. The lengths presented are held constant between both configurations. It is also helpful to show the RNEP to distinguish the areas of the entrainment duct and rocket.

In Figure 11 the RNEP of the Twin Circular configuration is shown. The rocket nozzles are above the entrained air area. The entrainment duct is where choking is desired to occur. 


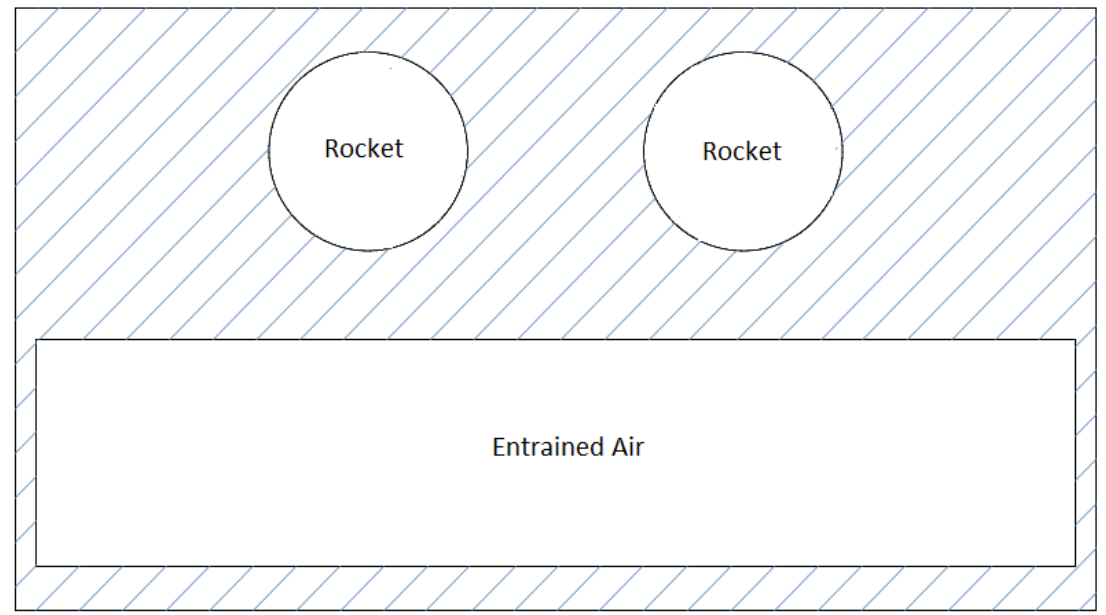

Figure 11: Twin Circular RNEP

\subsubsection{Exchange Inlet Geometry}

The Exchange Inlet geometry has two Exchange Inlet nozzles designed with 3 annular exit locations which accelerate flow to Mach 2.0. The nozzle geometry for the Exchange Inlet was designed to achieve the same operating conditions in terms of Mach number and mass flow as the Twin Circular nozzle. A close up of the nozzle is shown in Figure 8, Note that this particular design of the Exchange Inlet has 3 annular exhaust sections. The flow chokes at A and rocket exhaust exits at B.

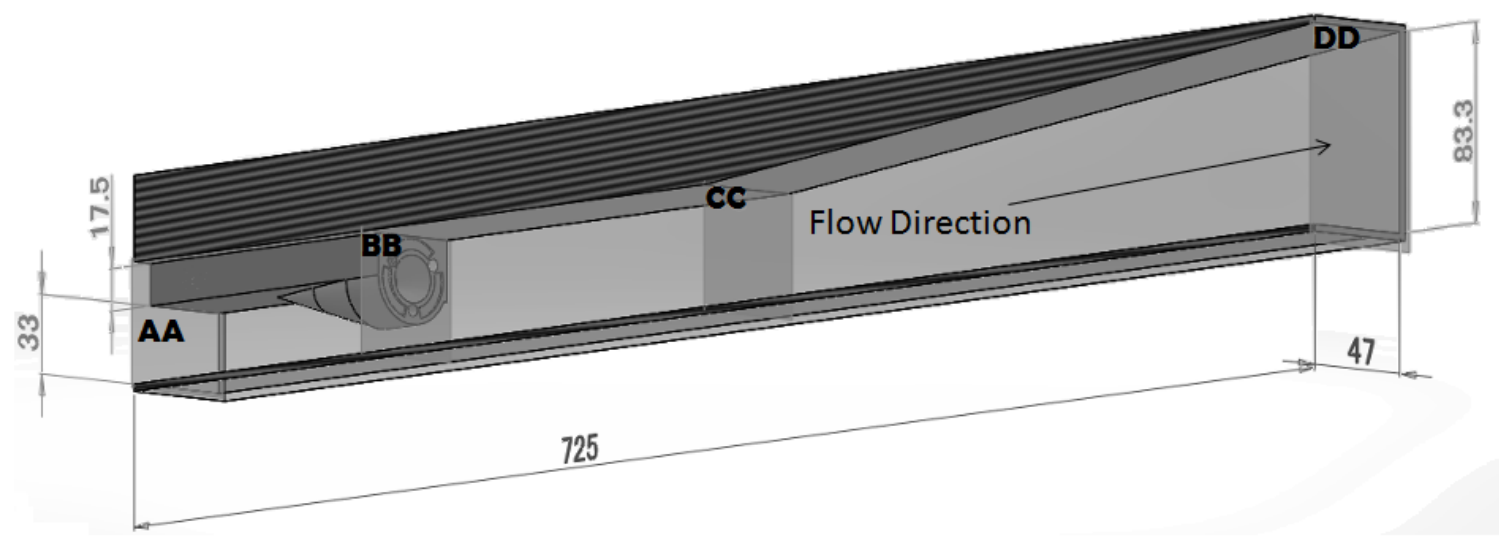

Figure 12: Exchange Inlet configuration 3D view 
In Figure 12 the Exchange Inlet nozzle is shown inside half of the rocket engine. Similar to Figure 10, Figure 12 shows the symmetry plane with the black hatched area and shows AA as the Entrainment Duct inlet, BB shows the RNEP, CC shows the ESP, and DD shows the EEP. The region between AA and BB is the entrainment duct, between $\mathrm{BB}$ and $\mathrm{CC}$ is the straight duct, and between $\mathrm{CC}$ and $\mathrm{DD}$ is the geometric expansion duct.

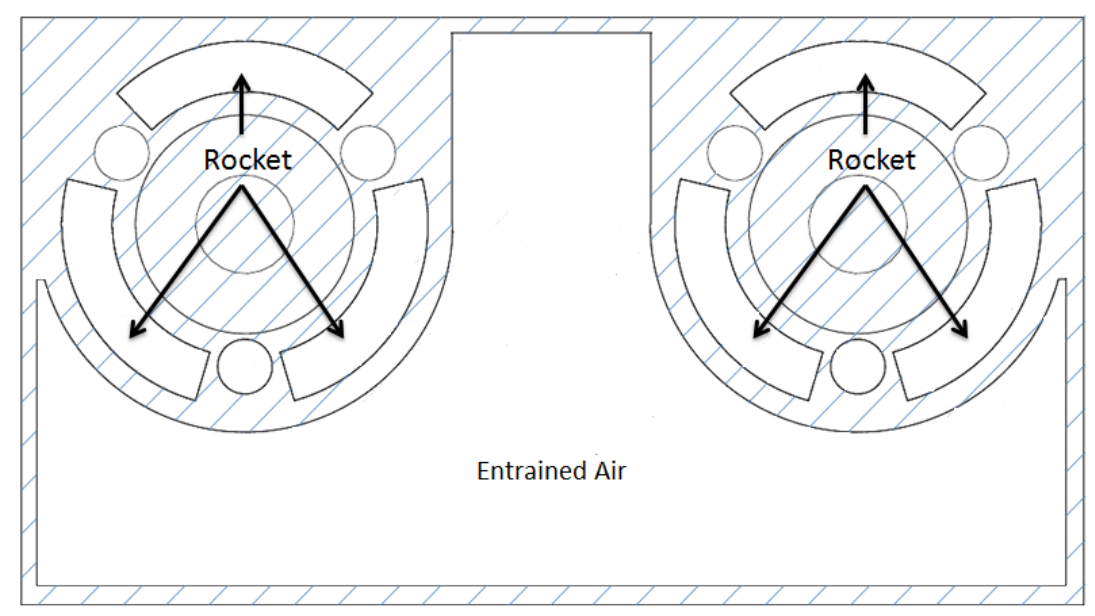

Figure 13: Exchange Inlet configuration RNEP

In Figure 13 the RNEP of the Exchange Inlet configuration is shown. It is significantly different than the Twin circular configuration seen in Figure 11, however, similarity is achieved between the two geometries by matching the ratio of Entrained air area and total flow area which includes rocket flow $\left(\theta=A_{a} /\left(A_{r}+A_{a}\right)\right)$ where $A$ is the area and the subscripts $a$ and $r$ represent air and rocket respectively. In addition to the large entrained air area are 6 circular holes present between the rocket exhaust sections which allow additional air to be entrained. The circular holes are entrainment tubes necessary to achieve similarity in the ratio of entrained air to total flow area.

The area ratios of both the Twin configuration and the the Exchange Inlet configuration are shown in Table 3 (each having an area ratio of 0.21 ). If $\sigma$ was set to 


\begin{tabular}{|l|l|l|l|}
\hline Engine configuration & Area Rocket $\left(\mathrm{mm}^{2}\right)$ & Area Entrained $\left(\mathrm{mm}^{2}\right)$ & Area Ratio $(\sigma)$ \\
\hline Exchange Inlet & 639 & 2416 & 0.21 \\
\hline Twin Circular & 508 & 1933 & 0.21 \\
\hline
\end{tabular}

Table 3: RNEP Area Properties

0 there would be no entrained air resulting in a pure rocket flow whereas increasing $\sigma$ increases the entrained area. If this ratio was not equal between the two configurations, an unfair comparison of $\dot{m}_{\text {air }}$ would result. Having a constant area ratio between both geometries allows for comparison based only on nozzle geometry.

\subsection{Numerical Setup}

The numerical setup of the engine is discussed here. The parameters of interest are the entrainment performance at the inlet of the engine, the outbound average Mach number of engine, as well as the average Oxygen concentration of the engine.

\subsubsection{Domain and Boundary Conditions}

The domain can be seen in Figure 14. The domain displayed is for the Exchange Inlet configuration, however the Twin Circular configuration is designed with the same values. In Figure 14 EL is the engine length, specified as $725 \mathrm{~mm}$. A symmetry plane is used along the face shown and no slip conditions are used along the surfaces within the rocket engine. The dashed box denotes the rocket engine. The tops and bottoms of the domain are free slip surfaces.

The inlet and outlet conditions are specified as $5 \mathrm{~m} / \mathrm{s}$ with a pressure specified at $101 \mathrm{kPa}$ and temperature of $292 \mathrm{~K}$. The conditions are typical sea level static conditions with a slight amount of momentum imparted into the domain. The rocket conditions were specified as pure Oxygen $\left(\mathrm{O}_{2}\right)$ at Mach 2.0. The simulations are 


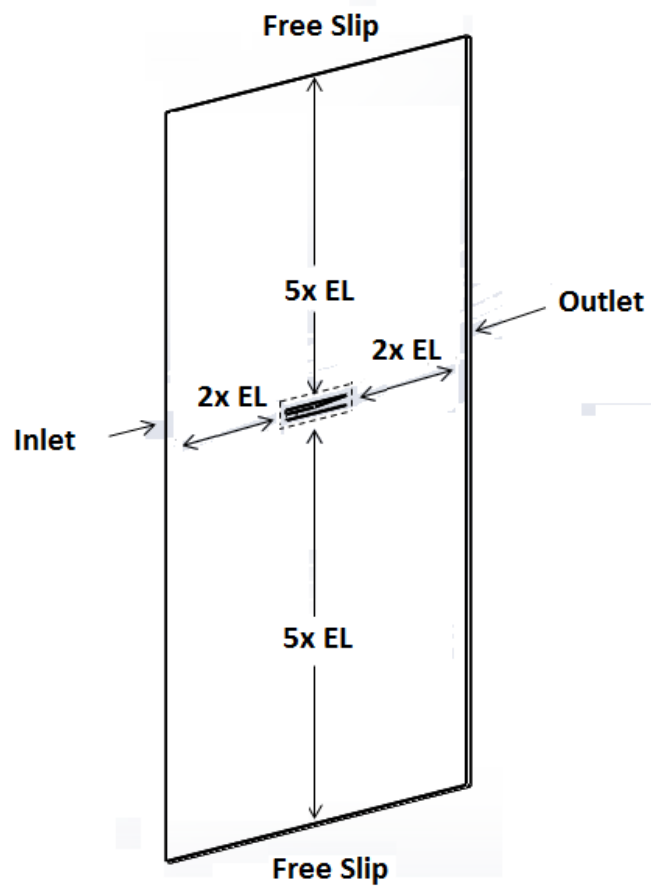

Figure 14: Domain of Exchange Inlet configuration

separated into low and high rocket pressure conditions. The low pressure condition is set near $750 \mathrm{kPa}$ while the high pressure conditions are specified near $1400 \mathrm{kPa}$. The nozzle exhausts are simplified as inlet planes and treated as constant velocity boundaries. The velocities, density, and temperature are specified on these inlet planes. The initialization conditions for the domain and rocket inlets are described in the following section.

\subsubsection{Initialization}

The domains are initialized with a velocity of $5 \mathrm{~m} / \mathrm{s}$ with a concentration of 21 $\%$ oxygen and $79 \%$ nitrogen comparable to air. This is to eliminate instabilities experienced at near zero velocity along the free stream inlet boundary. For the rockets, a constant velocity boundary condition is set based a nozzle designed for Mach 2.0 with a pure oxygen flow with the stagnation conditions of pressure $\left(P_{O}\right)$, temperature 


\begin{tabular}{|l|l|l|l|l|}
\hline Rocket Pressure & Configuration & $T_{O}(\mathrm{~K})$ & $\rho_{O}\left(\mathrm{~kg} / \mathrm{m}^{3}\right)$ & $P_{O}(\mathrm{kPa})$ \\
\hline Low & Exchange Inlet Long & 360 & 9.06 & 868 \\
\hline High & Exchange Inlet Long & 360 & 15.12 & 1415 \\
\hline Low & Twin Circular Long & 360 & 8.47 & 793 \\
\hline High & Twin Circular Long & 360 & 15.34 & 1436 \\
\hline Low & Exchange Inlet Short & 360 & 8.36 & 868 \\
\hline High & Exchange Inlet Short & 360 & 15.32 & 1434 \\
\hline Low & Twin Circular Short & 360 & 7.90 & 740 \\
\hline High & Twin Circular Short & 360 & 14.86 & 1391 \\
\hline
\end{tabular}

Table 4: Rocket Nozzle Stagnation Conditions

$\left(T_{O}\right)$, and density $\left(\rho_{O}\right)$ specified in Table 4 . In addition to the engine configuration displayed, engine configurations without the expanding section are considered and are called the short engine configurations.

For the higher nozzle pressure cases, the flow is initialized with the lower nozzle pressure cases to decrease computational time required to reach a solution.

\subsubsection{Residuals}

Convergence for these simulations is judged by 3 methods. The mass flow within the engine at various planes (one location $75 \%$ of the entrainment duct, the RNEP (BB), ESP (CC), and EEP (DD)), a maximum residual calculated for the entire domain, as well as a set of residuals calculated for the area within the engine (also seen in Figure 14 as the area within the dotted lines). Grid convergence is considered separately and done for the Exchange Inlet geometry and at only the lower pressure. 


\section{Mass Flows}

The planes that are examined are the plane $75 \%$ of the entrainment tube, the RNEP, the beginning of the expansion section and at the engine exit plane. When these planes are converged to within 3 orders of magnitude the flow is considered converged with respect to mass flow.

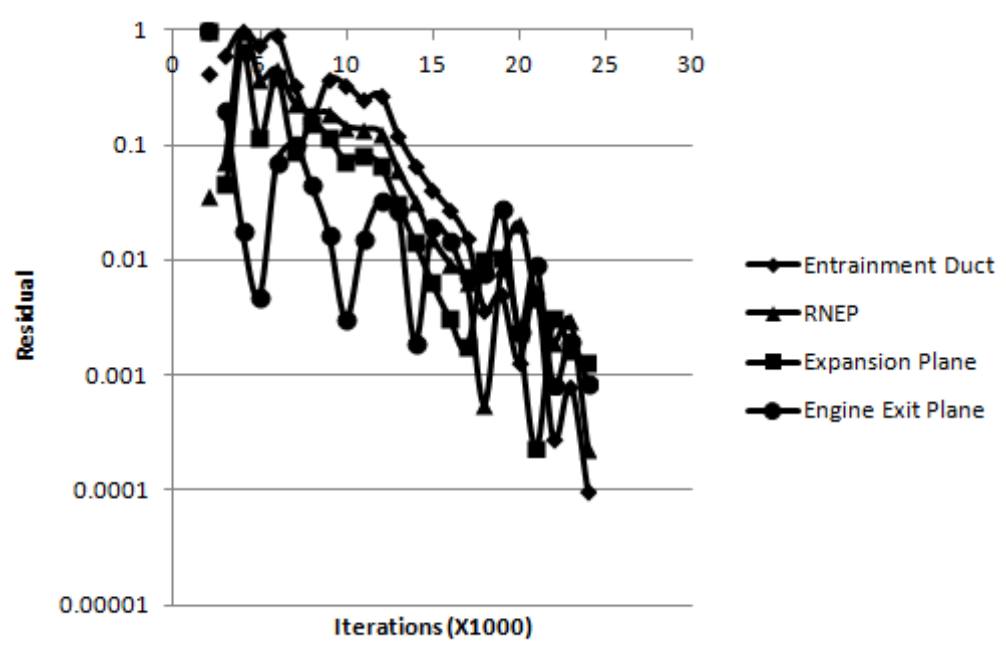

Figure 15: Exchange Inlet configuration Mass flow Residuals

Figure 15 shows the mass residuals for the low pressure Exchange Inlet configuration. After 24,000 iterations, all of the planes sampled show a converged solution in terms of mass flow.

\section{Residuals}

The residuals from the software package itself over the whole domain converge by 1 order of magnitude. The residuals calculated automatically by the software package sum the delta values of all calculated values over the entire domain where large portions of the domain do not change (i.e. they are set at freestream values which remain stable). The local residuals are calculated based on the change of the individual primitive variables of the flow inside the engine seen in Figure 14 as the area within 
the dashed lines. While the flow over the whole domain only converges by 1 order of magnitude, the flow in a local region within the engine converges by 3 or 4 orders of magnitude. For the local convergence, the primitive variables are normalized against the maximum difference for the variable.

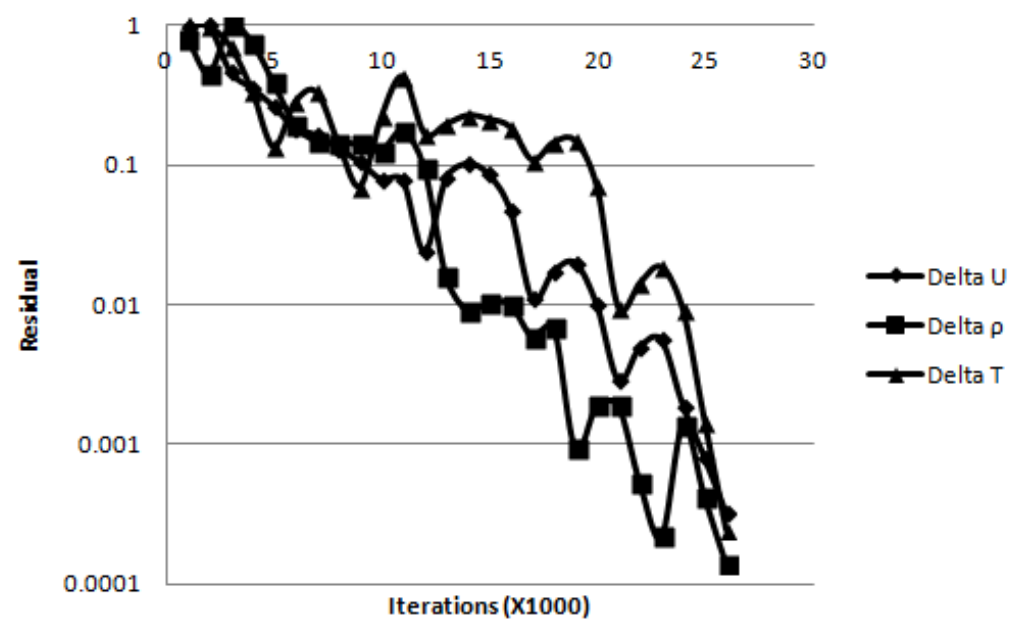

Figure 16: Exchange Inlet configuration local residuals

Figure 16 shows the local residuals for the low pressure Exchange Inlet long configuration and shows that for all primitive variables the local area is converged to 3 orders of magnitude after 26,000 iterations and Figure 17 shows residuals output by the software package over the whole domain. 


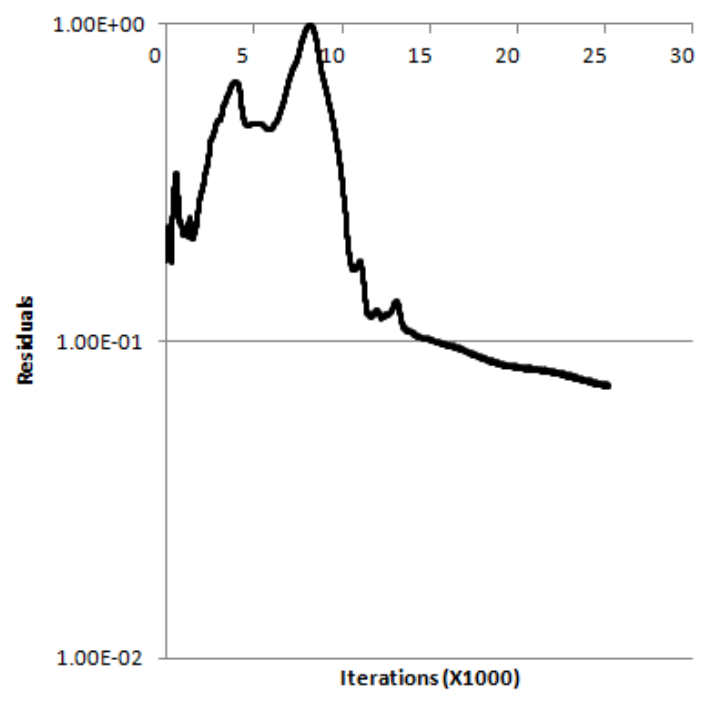

Figure 17: Software Package Residuals

\begin{tabular}{|l|l|l|}
\hline Node Count & Inflation layers & expansion factor of inflation \\
\hline 800,000 & 20 & 1.21 \\
\hline $1,250,000$ & 18 & 1.2 \\
\hline $2,000,000$ & 18 & 1.2 \\
\hline
\end{tabular}

Table 5: Grid Sizing

\section{Grid Convergence}

Grid convergence is conducted for the Exchange Inlet long engine configuration at the low pressure. The parameters for the three grids used are shown in Table 5.

The reason for some of the discrepencies between inflation layers and expansion factor is due to difficulty with grid generation. It was found that with certain grid configurations, there were inconsistencies in the grid and iterative input was used to find a set of specifications that worked, hence the higher node count grids having less inflation layers. However a benefit of this is that the grid near the areas of interest (central in the engine) contain more nodes.

As an example, Figure 18 shows the medium grid used for the refinement study. 
It is seen that the grid sizing is relatively uniform within the engine. Areas around edges and corners were given higher node distribution resulting in the more densely meshed areas near the edges.

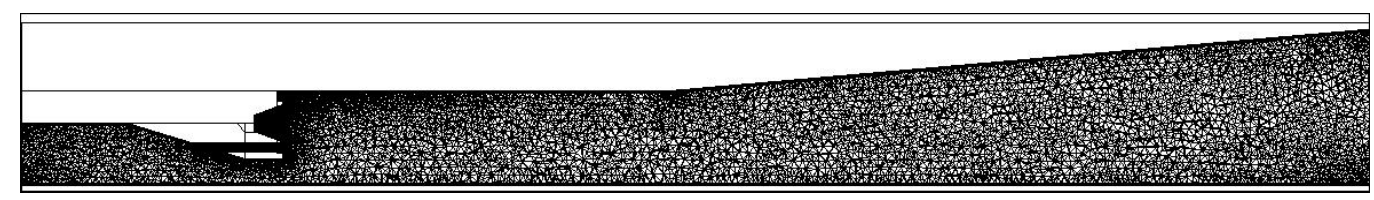

Figure 18: Medium Grid within the Exchange Inlet

Figure 19 shows a close up near the nozzle location where a higher density of mesh elements exists. This figure also shows the inflation layers within the engine.

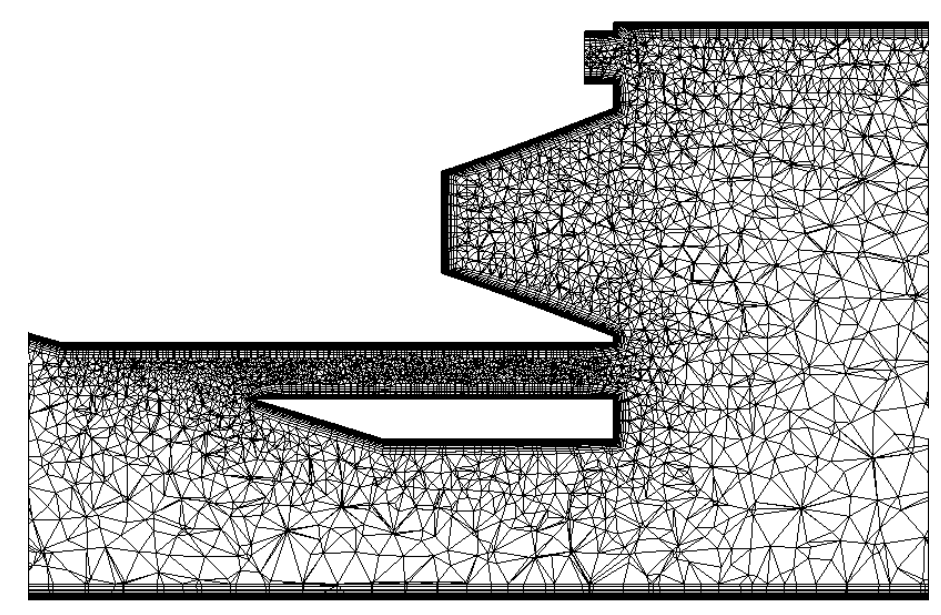

Figure 19: Medium grid near Exchange inlet nozzle

The grids were compared based on a line along the lower surface inside the engine directly below the nozzle. The results are shown in Figure 20.

Figure 20 shows that as the node count increases there is less change with each iteration in the pressure. The grids are increased based on an approximately 1.5 scaling increase in nodes. The minimum pressure between all 3 grids changes at most $15 \%$. The difference between the low and medium refinement is $15 \%$ while the difference between the medium and highly refined grids is $3 \%$. Therefore the 


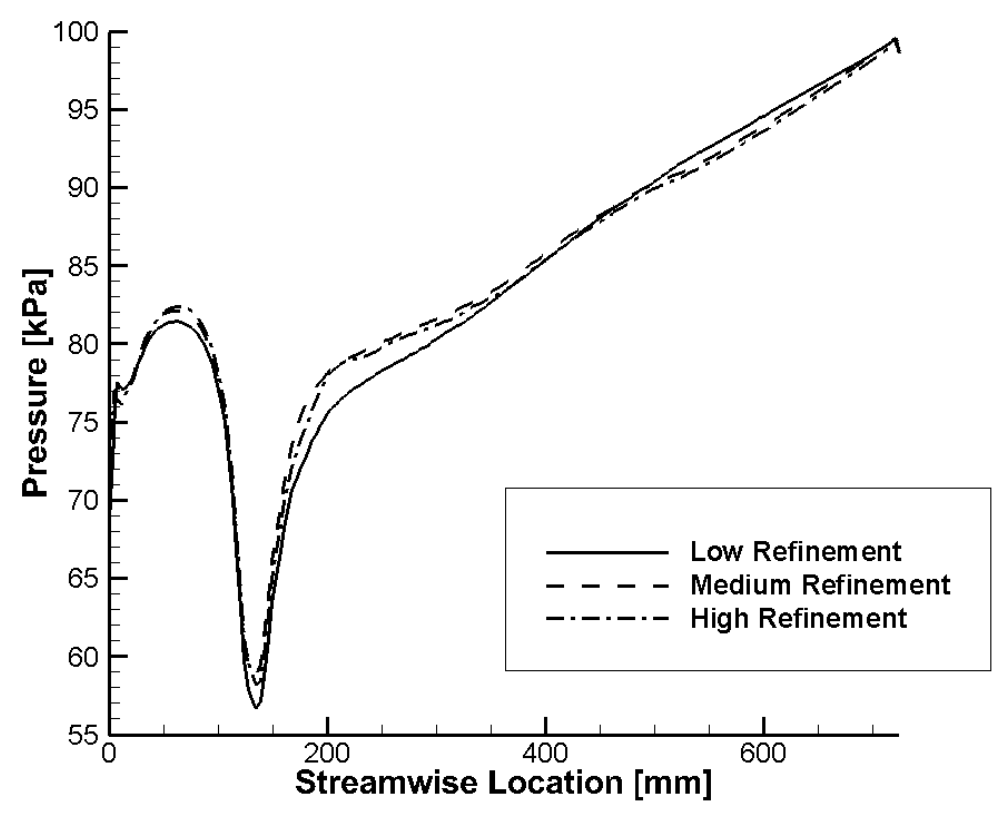

Figure 20: Grid Refinement Study [Pressure]

medium grid is found acceptable based on the pressure criteria.

Another method to confirm grid convergence is to investigate the velocity profile at a location downstream of the nozzle. The location chosen was in the expanding section inline with the rocket nozzle and halfway along the expanding section in the streamwise direction. Figure 21 shows how the velocity profile in this line compares between the different grids.

Figure 21 shows the streamwise velocity for each of the grids generated. It can be seen in Figure 21 that as the node count increases there is a change in the velocity profile with each grid. All three profiles follow a similar trend, however the peak velocity and location change slightly between each grid refinment. The increase from the low to medium grid is $3.6 \%$ while the increase from medium to high refinement is $0.8 \%$. Therefore the medium grid is found to be acceptable. 


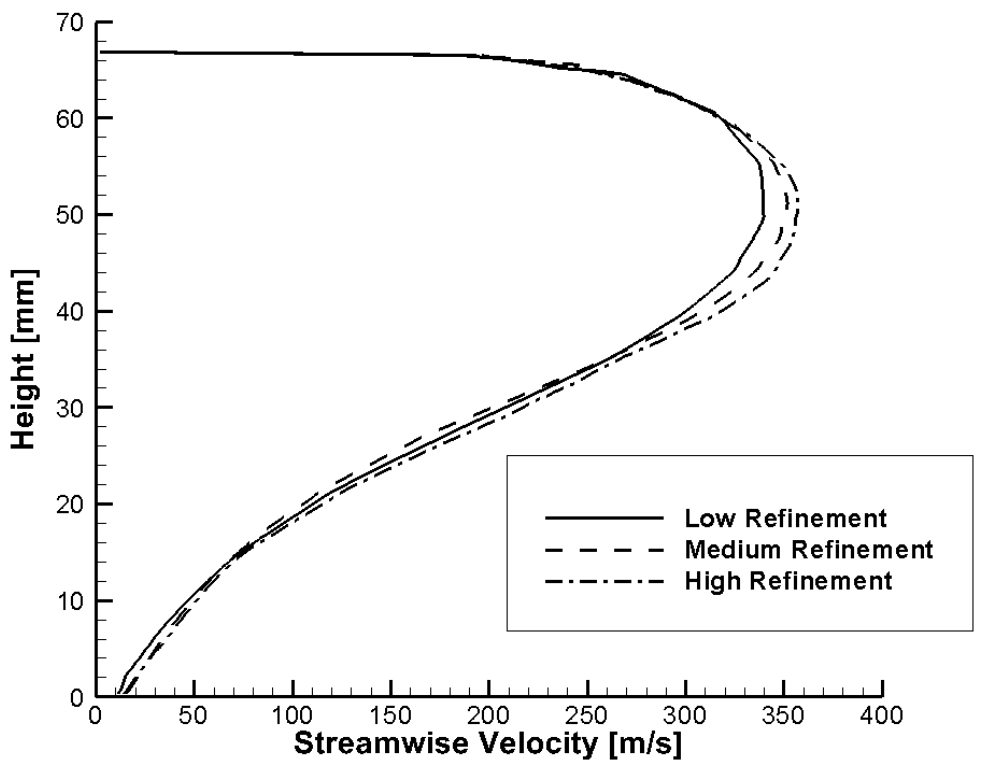

Figure 21: Grid Refinement Study [Velocity] 


\section{Chapter 4}

\section{Results}

The computational results are examined and an assessment is made of the two nozzle configurations. The Exchange Inlet configuration will be examined first followed by the Twin circular configuration. The twin configuration is used much like a benchmark as it is one of the design choices for RBCC engines by the Japanese Space Agency. The Exchange Inlet serves as a novel alternative to the nozzle types studied by JAXA.

\subsection{Validation}

To assess the prediction capabilities of the numerical study, a validation case is conducted. An experiment was conducted by a research group at JAXA in 2011 [46]. The experiment consisted of a test bed with a total of 84 pressure taps distributed within geometry described in Chapter 3. From the data, a pressure distribution is given along the upper and lower walls within the geometry. In the experiment, pure oxygen is also used as the rocket flow. In the experiment, a range of stagnation pressures between $500 \mathrm{kPa}$ and $1500 \mathrm{kPa}$ are used for the rocket flow. Additionally, 2 nozzles exist in the experiment where numerically this is simulated with a symmetry

plane. The pressure taps are streamwise along the centerplane as well as $28 \mathrm{~mm}$ offset 
from the centerplane. The data for the experimental study following the same geometry and flow conditions (free stream atmospheric air, pure $\mathrm{O}_{2}$ rocket flow, $\mathrm{M}=2$ ) proposed in Chapter 3 for both the Exchange Inlet and Twin circular configuration at the lower pressure of $P_{O}=868 \mathrm{kPa}$ and $793 \mathrm{kPa}$ respectively is used as comparison for the numerical data. From the experimental study, the data points collected along the symmetry plane of the engine are used to compared to the numerical study.

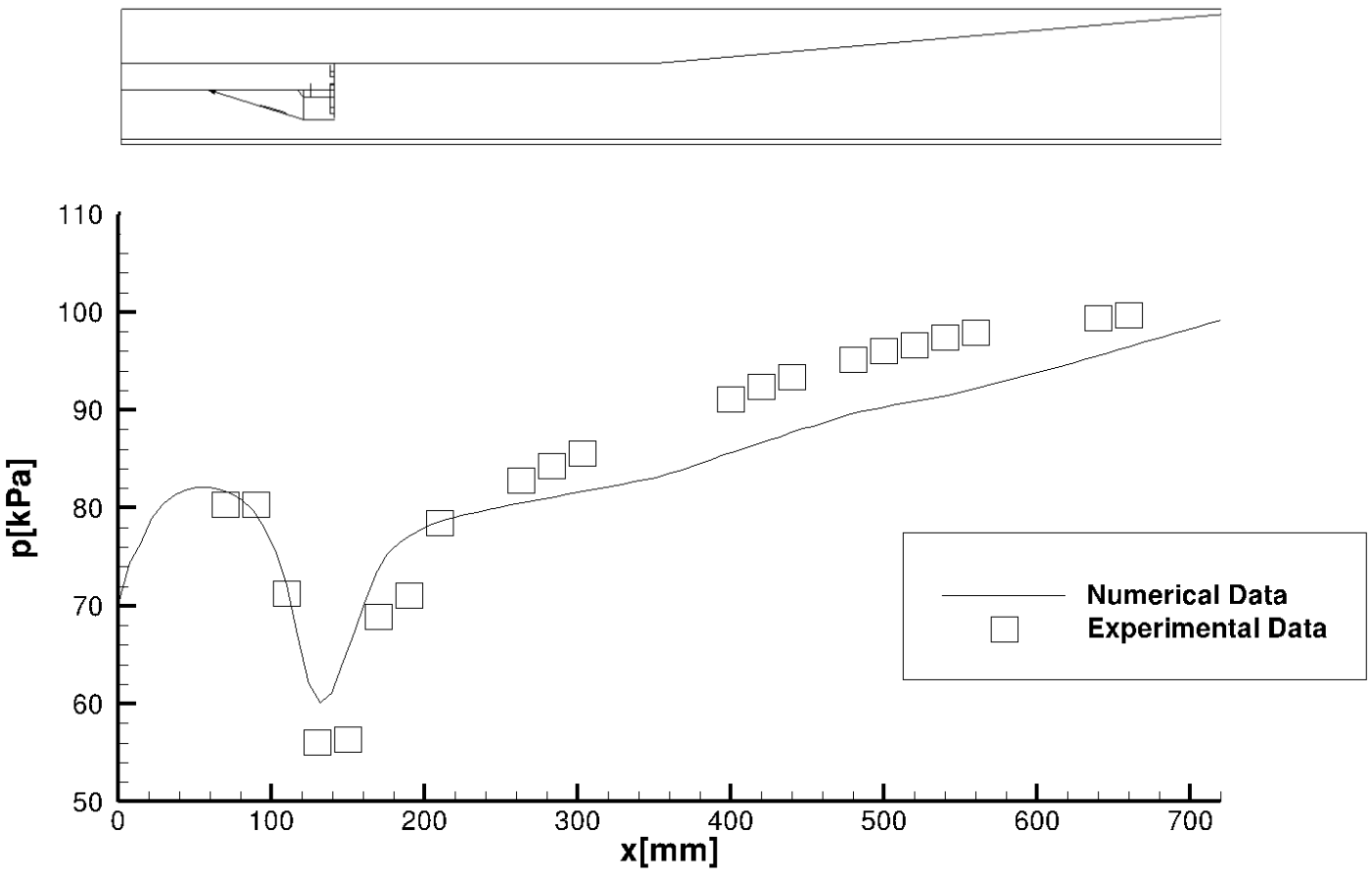

Figure 22: Exchange inlet lower surface pressure plot

It can be seen in Figure 22 that for the Exchange Inlet configuration the pressure plot along the lower surface shows good agreement between the experimental and numerical data. The greatest deviation between experimental and numerical data is $6.7 \%$ at the location just after the expansion duct begins $(400 \mathrm{~mm})$. The difference at the low peak value underneath the RNEP is $1.7 \%$ at the location $140 \mathrm{~mm}$ of the engine. The average difference between the numerical and experimental data is $4 \%$. The strong agreement between the experimental and numerical data gives confidence 
in the software's ability to predict the flow within this geometry.

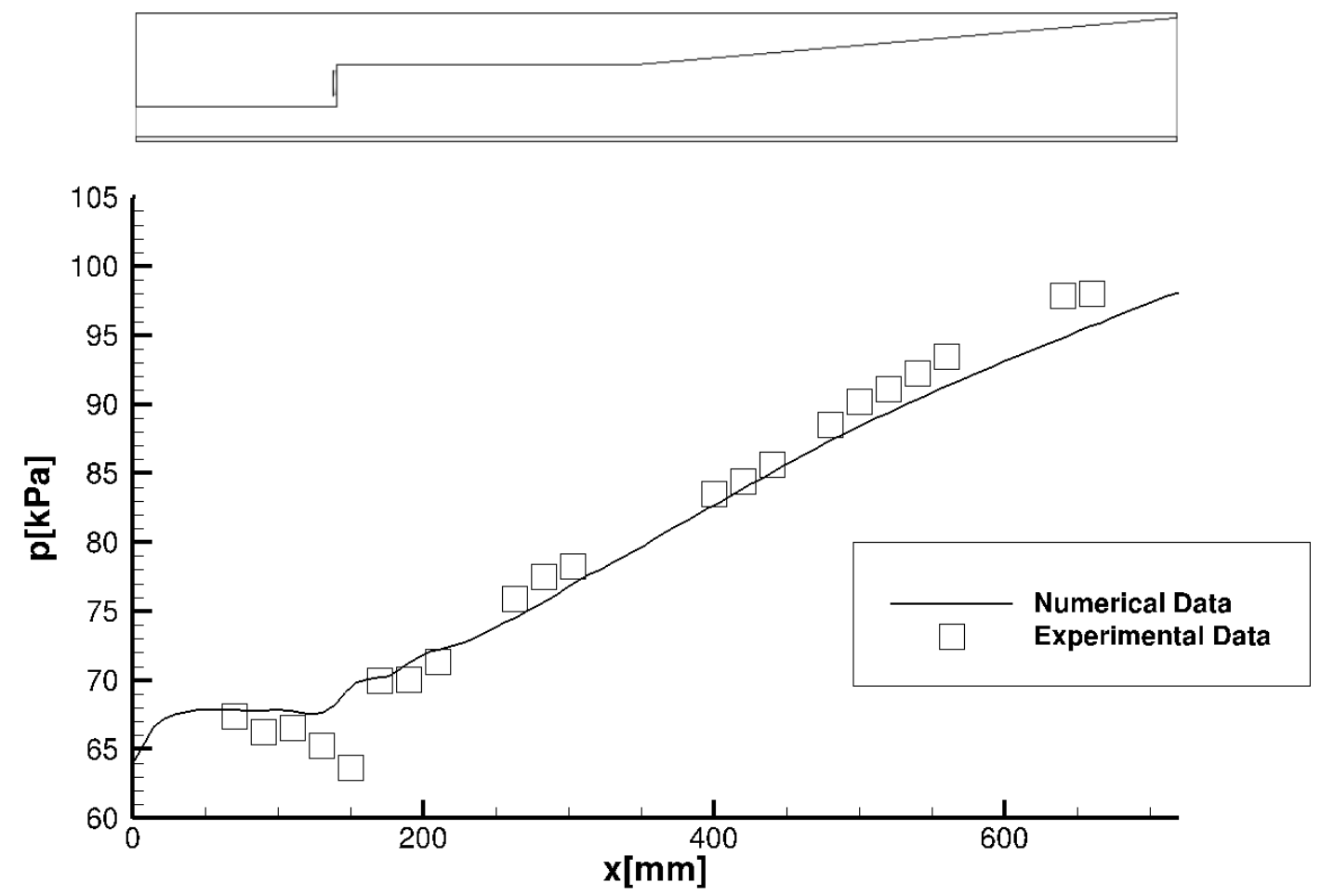

Figure 23: Twin circular pressure plot along lower surface

The Twin circular configuration is also compared numerically and experimentally. Figure 23 shows the pressure distribution along the lower surface of the Twin circular configuration. The most significant difference between the numerical and experimental data is at the RNEP, here the experimental results show an expansion or acceleration of the flow along the rocket flow plane. This amounts to an error of $6.2 \%$ based on the minimums in the location which is also the maximum difference. Similar to the Exchange Inlet in Figure 22, the experimental flow has lower pressure prior to the rocket nozzle but higher pressure shortly after. This indicates that the mesh has difficulty accurately capturing the acceleration and deceleration that is happening around the nozzle location. However, the average difference between the numerical and experimental data is $1.5 \%$. As with the Exchange Inlet configuration, 
good agreement is found between experiment and numerical analysis.

\subsection{Full Engine Configuration}

The full engine configuration is described as is shown in the Geometry section. An entrainment section of $140 \mathrm{~mm}$ allows the free stream flow to be drawn into the engine with the desire to achieve choked flow, allowing for maximum entrainment. From the RNEP, a straight duct is followed for $210 \mathrm{~mm}$ at which point an expansion occurs. At a length $350 \mathrm{~mm}$ into the engine, the expansion allows the flow to expand and mix further until it reaches the exit plane located $725 \mathrm{~mm}$ downstream.

\subsubsection{Low Pressure Cold Gas Configuration}

The low pressure cold gas configuration refers to the Exchange Inlet and Twin circular configurations with the pressures of $868 \mathrm{kPa}$ and $796 \mathrm{kPa}$ respectively. Since the two pressures differ only slightly, these two cases will be compared to one another. The numerical data examined are the pressure plots along the lower and upper surface of the two configurations, a Mach plane at the RNEP (BB) and ESP (CC), a Mach and $\mathrm{O}_{2}$ partial density plane at the $\mathrm{EEP}(\mathrm{DD})$, streamwise pressure and $\mathrm{O}_{2}$ partial density centered on the rocket nozzle through the engine, as well as stream line traces. Numerical results are only obtained for a half engine simulation therefore data is mirrored about the symmetry plane on the ESP and EEP for accurate representation.

\section{Wall Pressures}

Figure 24 shows a streamwise pressure line centered underneath the rocket nozzle (28 mm offset from the symmetry plane) as well as a pressure line along the symmetry plane. The Exchange Inlet configuration shows a significant decrease in pressure at the $140 \mathrm{~mm}$ location. The pressure can be related to the Mach number of the flow, 


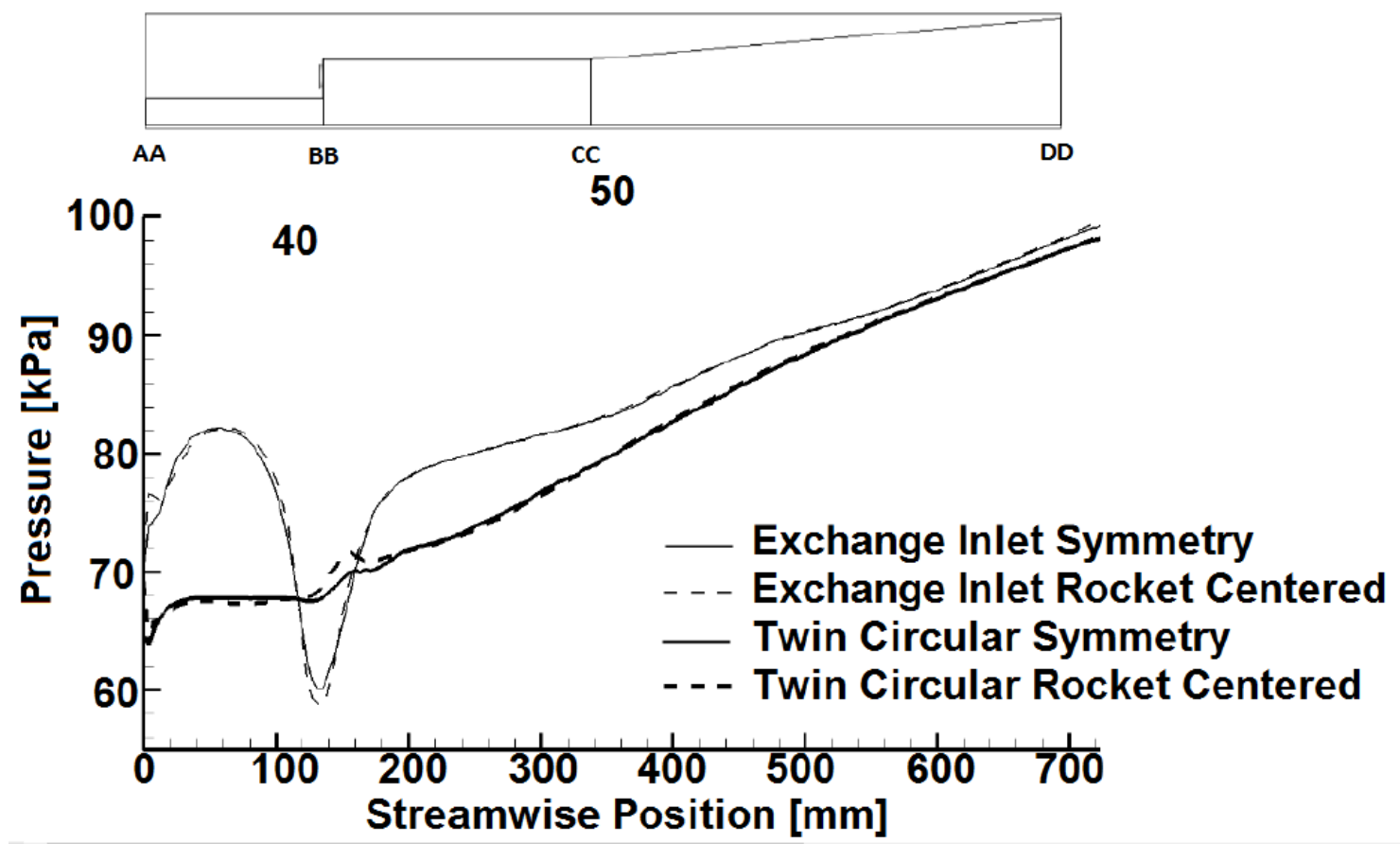

Figure 24: Lower Surface Low Pressure Distribution for Long Engine configuration

a low pressure is equivalent to a high Mach number. The pressures can be related to the Mach number through the equation

$$
M=\sqrt{\frac{2}{\gamma-1}\left({\frac{P_{o}}{P}}^{\frac{\gamma-1}{\gamma}}-1\right)}
$$

which for the entrained air has values for $P_{O}=101 \mathrm{kPa}$ and $\gamma=1.4$. At a pressure of $52.8 \mathrm{kPa}$, air is considered Mach 1.0. The pressure seen in the Exchange Inlet configuration reaches a minimum of $59.7 \mathrm{kPa}$ corresponding to $\mathrm{M}=0.9$. The Twin circular configuration does not show the same kind of decrease. A minimum pressure of $68.2 \mathrm{kPa}$ corresponding to $\mathrm{M}=0.77$ is experienced at the same location in the Twin configuration. The lower pressure in the Exchange Inlet indicates that the Exchange Inlet is closer to a choked condition than the Twin circular configuration. The pressure line along the symmetry plane shows little deviance from the line centered underneath the rocket for both the Exchange Inlet and Twin circular configuration. This indicates 
that in both the Exchange Inlet and Twin configurations, the flows are uniformly accelerated along the lower surface.
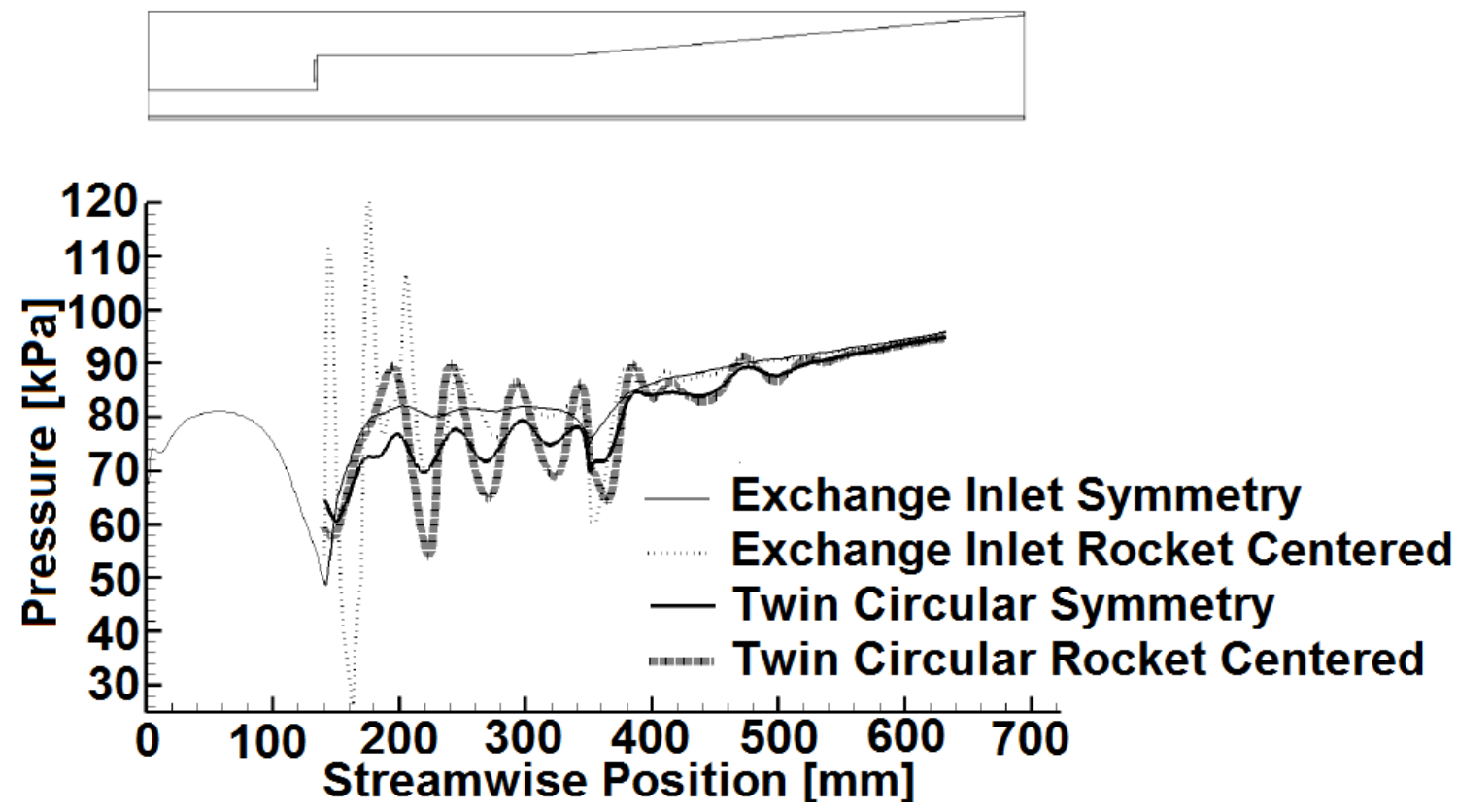

Figure 25: Upper Surface Low Pressure Distribution for Long Engine configuration

The upper wall pressures are shown in Figure 25. The Exchange Inlet symmetry plane shows data from Streamwise Position $=0$ to $725 \mathrm{~mm}$ because there is a separation between the two nozzles allowing for a flat upper wall from which data can be collected. The pressure along the symmetry plane does not show any significant oscillation indicating no shocks along this line. The streamwise line centered on the rocket shows strong initial oscillation in the Exchange Inlet configuration but quickly dissipates. The sharp peaks along this line indicate a shock train which is when flow expands and compresses rapidly due to supersonic flow alternating between over and under-expanding due to momentum. This is expected as the line follows the rocket path closely. At the expansion plane $350 \mathrm{~mm}$ downstream, there is a sudden decrease in pressure as a result of the supersonic flow. The oscillations are dissipated by the $450 \mathrm{~mm}$ location. Comparatively, the Twin circular configuration shows oscillations along both the symmetry line and the rocket centered streamwise line. The strong 
oscillations again indicate a shock train but do not dissipate at the same rate as the Exchange Inlet. The oscillations are dissipated by the $550 \mathrm{~mm}$ location.

\section{RNEP and ESP}

The $140 \mathrm{~mm}$ location is where choking is desired to occur. A choked flow as a result of the duct geometry results in higher mass flow rates than if flow is choked by a Fabri choke. In both the Exchange Inlet and Twin configuration, choking was not found based on Figure 24. Examining the RNEP gives further insight into the flow at this location.

Figure 26 shows the Mach contours at the RNEP of the low rocket chamber pressure simulations for both configurations. Figure 26 (a) shows RNEP of the Exchange Inlet configuration. In this region, there are some areas of where the entrained air reaches Mach 1. The entrainment tubes alone reach a choked condition. The flow approaches Mach 1 in many areas that are close to the nozzle but flow far from the nozzle tends to remain slower. Over half of the entrained flow reaches a Mach 0.8. At this plane, the entrainment ratio can be calculated. Summing the mass flow of the finite elements of the entrained air area coupled with the entrainment tubes results in a mass flow of $0.258 \mathrm{~kg} / \mathrm{s}$ which yields an entrainment ratio of $0.804\left(\dot{m}_{\text {air }} / \dot{m}_{r}\right)$.

In Figure 26 (b) the RNEP of the Twin circular configuration is shown. The entrained flow never accelerates to Mach 1 anywhere within this plane. The flow reaches a maximum of Mach 0.83 close to the upper wall of the entrainment duct. The entrained flow tends to reach high Mach numbers along the upper surface of the entrainment duct while the flow along the lower surface stays slow. The square entrainment duct shows weaknesses in entrainment in the corners as flow remains slow along the outer corners. The flow greater than Mach 0.8 is centered below the two rocket nozzles. The flow is also found to be uniform along the lower surface. The mass flow rate of air and entrainment ratio are found the same way for the Twin 


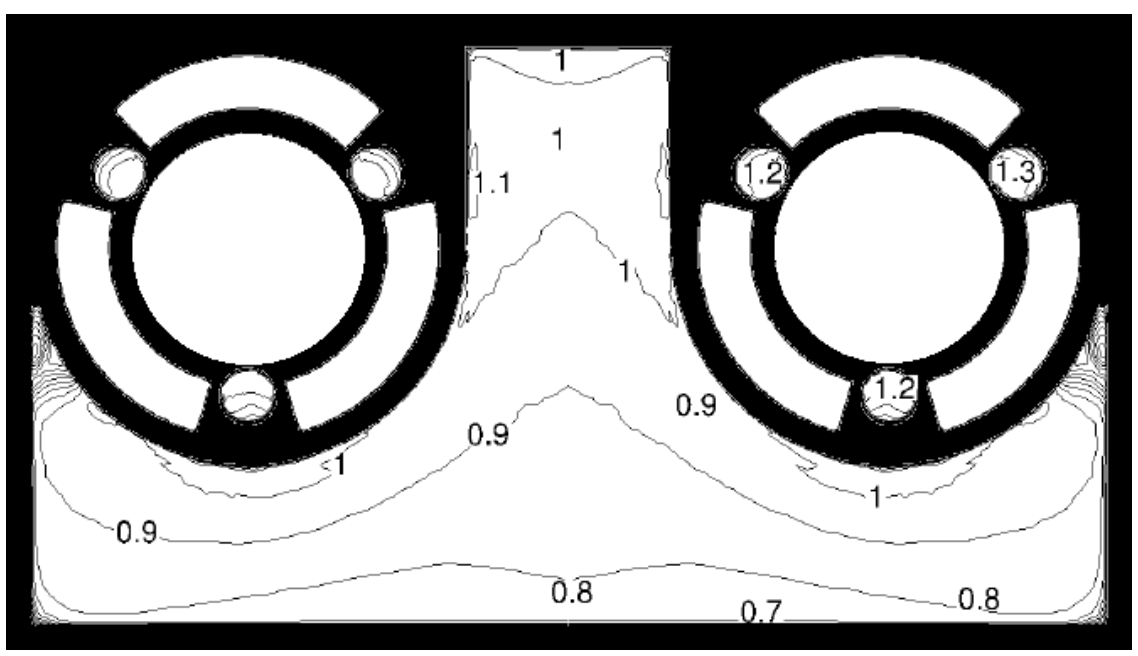

(a) Exchange Inlet

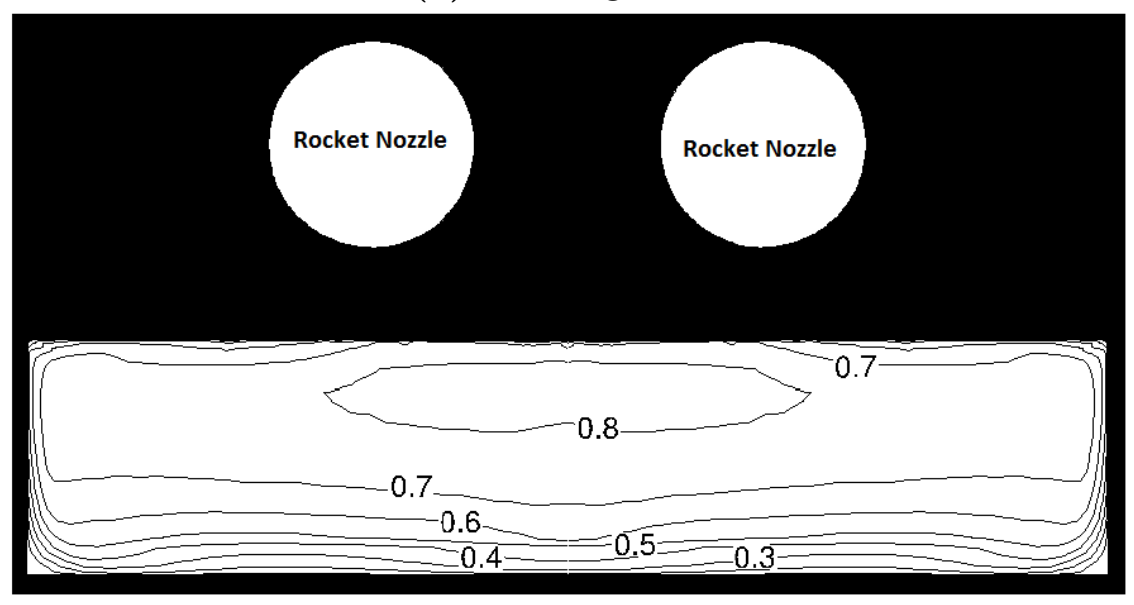

(b) Twin Circular

Figure 26: Low Rocket Chamber Pressure RNEP Mach Contours

circular configuration as it is in the Exchange Inlet configuration and they are found to be $0.172 \mathrm{~kg} / \mathrm{s}$ and $0.7319\left(\dot{m}_{\text {air }} / \dot{m}_{r}\right)$ respectively.

The ESP shows how the rocket core develops through this location. In both the Exchange Inlet configuration and Twin circular configuration, the pressure plots indicated supersonic flow at this location by virtue of a sharp decrease in upper wall pressure.

Figure 27 (a) shows the Mach distribution of the flow at the ESP of the Exchange Inlet. It is seen that a significant amount of the flow in the upper corners exceeds 


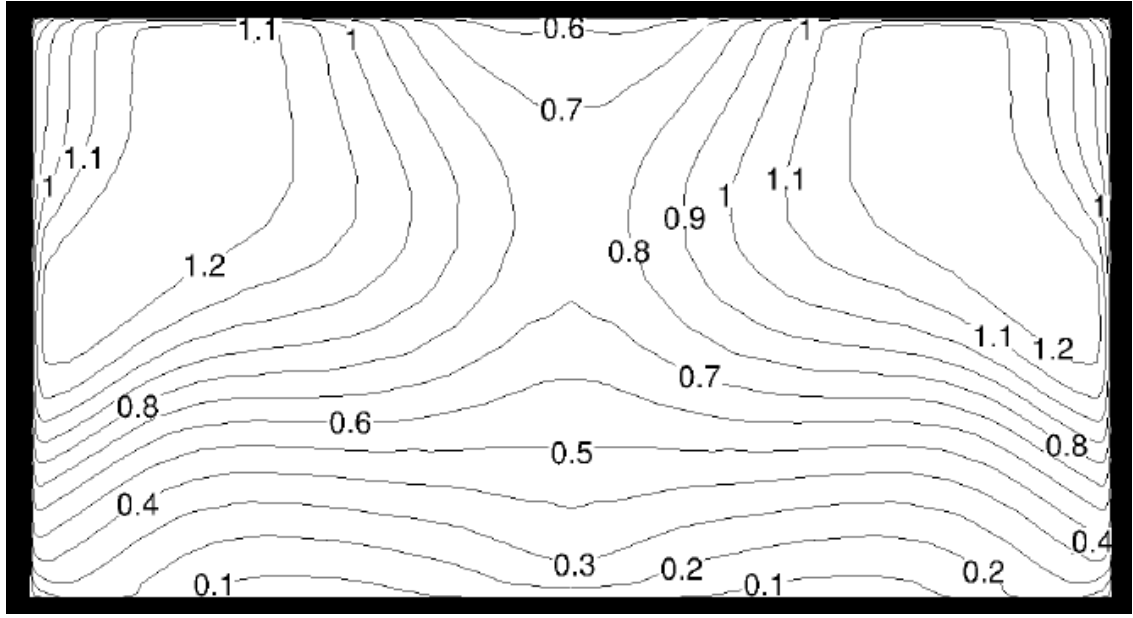

(a) Exchange Inlet

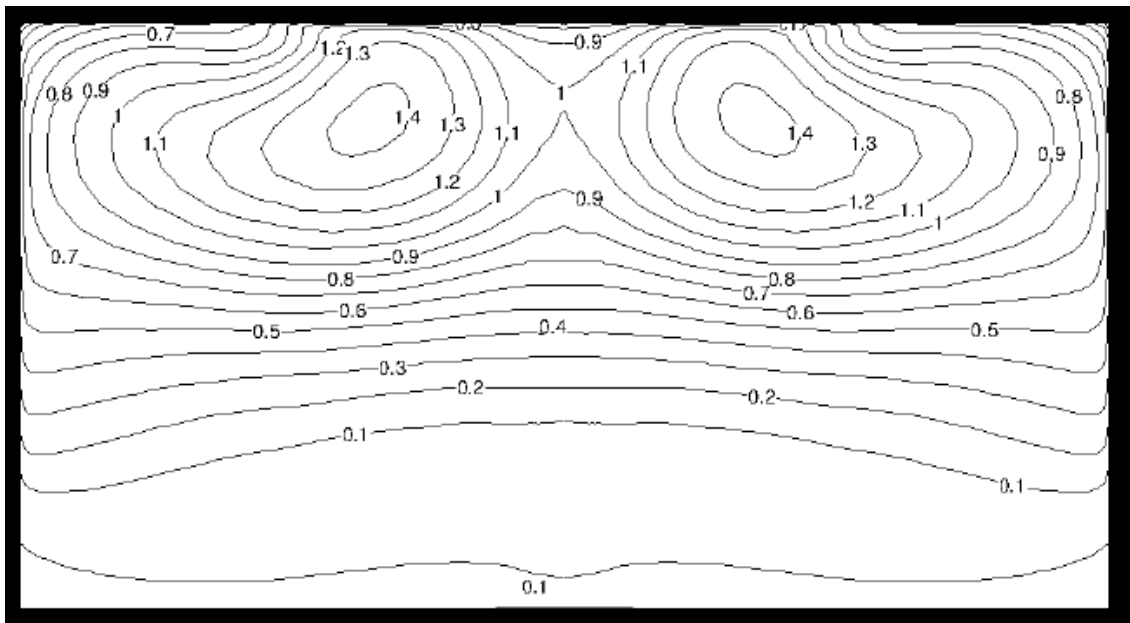

(b) Twin Circular

Figure 27: Low Rocket Chamber Pressure Mach Contours at ESP 
Mach 1 explaining the expansion of pressure in Figure 24. The flow tends to follow the region along the outer walls resulting in less acceleration along the symmetry plane. In this figure the region that falls below Mach 0.1 is where a small area of separation occurs. The Twin circular configuration is seen in Figure 27 (b) for comparison.

Figure 27 (b) shows that the rocket core from the nozzle is still evident at this location. The lower surface sees much slower flow in the Twin circular configuration than the Exchange Inlet configuration. The rocket core indicates that the rocket flow is not mixing as well with the entrained stream compared to the Exchange Inlet. The high Mach flow seen along the upper surface supports the existence of the shock train seen in the Twin circulars pressure lines from Figure 24. Along the lower surface, separation occurs along the width of the engine in a very thin region resulting in some recirculation.

\section{EEP}

The EEP show the final Mach distributions and the $\mathrm{O}_{2}$ composition of the rocket engines.

Figure 28 (a) shows the Mach distribution at the EEP of the Exchange Inlet configuration. The higher Mach flow remains along the outer walls of the engine as was seen in Figure 27 (a). The peak Mach number is 0.932 . The flow along the symmetry plane is not accelerated as significantly as the flow along the outer walls. At the symmetry plane near the lower surface there is a small region of flow below Mach 0.1 where some recirculation exists. The average Mach number based on area was found to be 0.415 . The Twin circular configuration is presented to in Figure 28 (b).

The Mach contours at the EEP of the Twin circular configuration are seen in Figure 28 (b). The rocket core still appears in the EEP near the symmetry plane and along the upper surface. The flow is much slower and more concentrated near the 


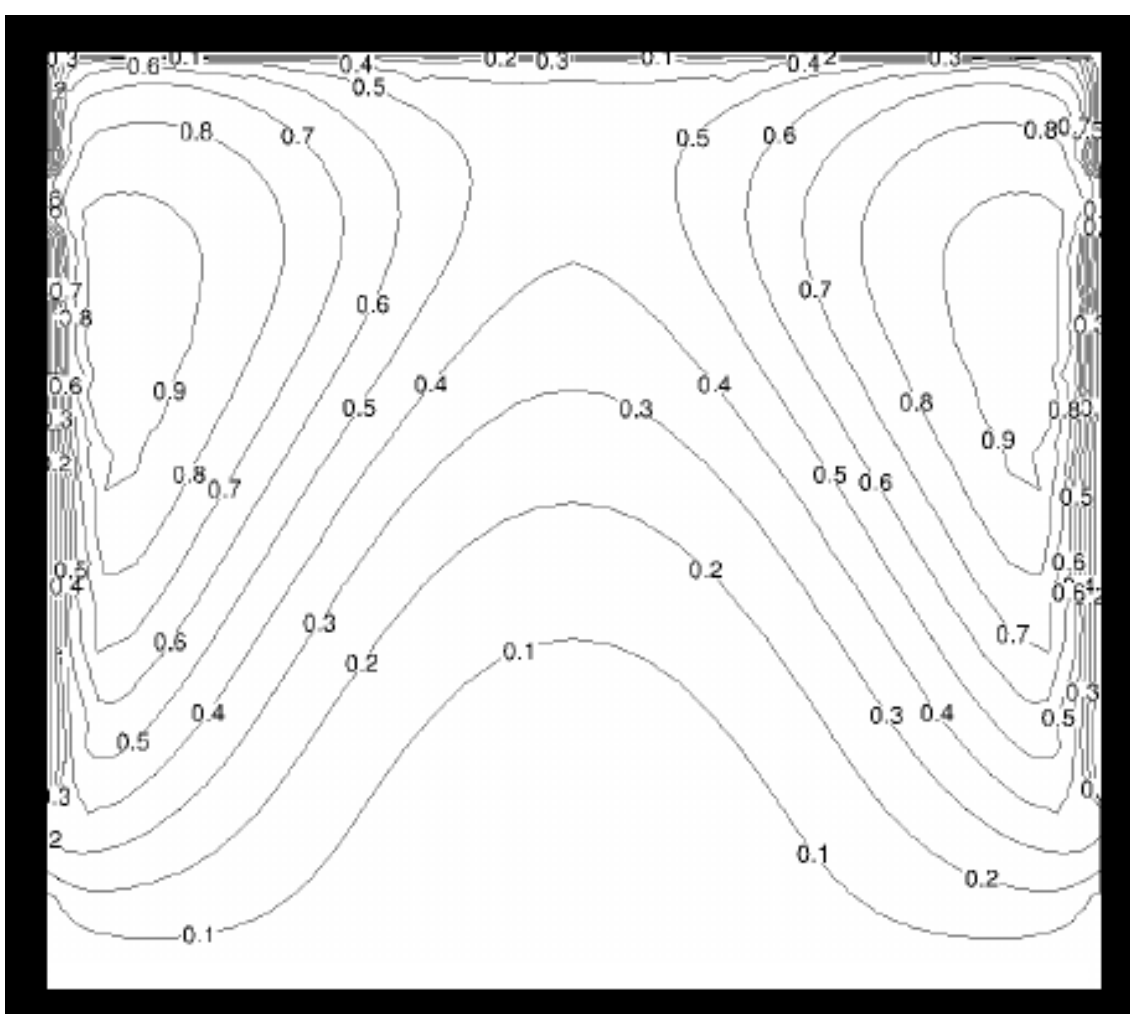

(a) Exchange Inlet

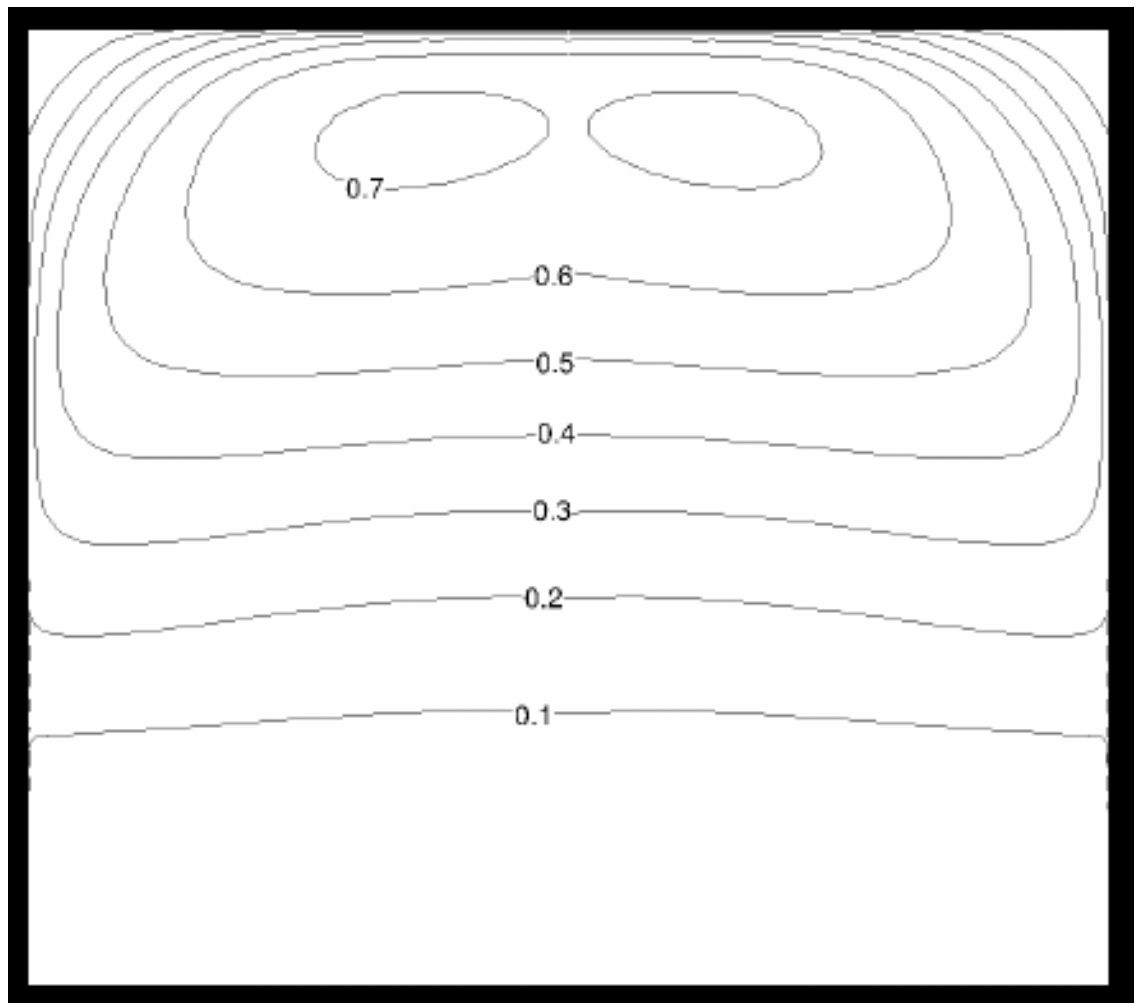

(b) Twin Circular

Figure 28: Low Rocket Chamber Pressure Mach Contours at EEP 
upper half of the EEP with a peak Mach number 0.78. The average Mach Number is 0.3. To assess how well the rocket flow mixes with the entrained air, the $\mathrm{O}_{2}$ partial densities at the EEP are examined.

The EEP $\mathrm{O}_{2}$ partial density of the Exchange Inlet is shown in Figure 29 (a). The nozzle exhaust is simulated as $100 \%$ pure $O_{2}$ and the entrained air is $21 \%$ $\mathrm{O}_{2}$, therefore any increase in partial density represents some of the pure rocket $\mathrm{O}_{2}$ mixing with entrained air. However very high partial densities of $\mathrm{O}_{2}$ indicate nearly pure rocket flow still existing (ie. not mixing with the entrained air). The average $\mathrm{O}_{2}$ partial density is $57 \%$. A peak value of $92 \%$ is found near the outer wall. The majority of the plane has an $\mathrm{O}_{2}$ partial density above $45 \%$ while at the center on the lower and upper surfaces some lower partial density regions exist. The minimum $\mathrm{O}_{2}$ partial density that can exist is $21 \%$ as this represents the entrained air. The lowest $\mathrm{O}_{2}$ partial density at the exit plane is $24 \%$. The Twin circular configuration shows a different trend than the Exchange inlet configuration with the $\mathrm{O}_{2}$ partial density being higher near the symmetry plane instead of the outer walls.

Figure 29 (b) shows the $\mathrm{O}_{2}$ partial density of the Twin circular configuration. The average $\mathrm{O}_{2}$ partial density is $55.8 \%$. The peak value is over $65 \%$ and the minimum is $28 \%$. The $\mathrm{O}_{2}$ partial density tends to be focused near the rocket core. The $\mathrm{O}_{2}$ partial density gradually decreases from the top of the EEP to the lower surface. 


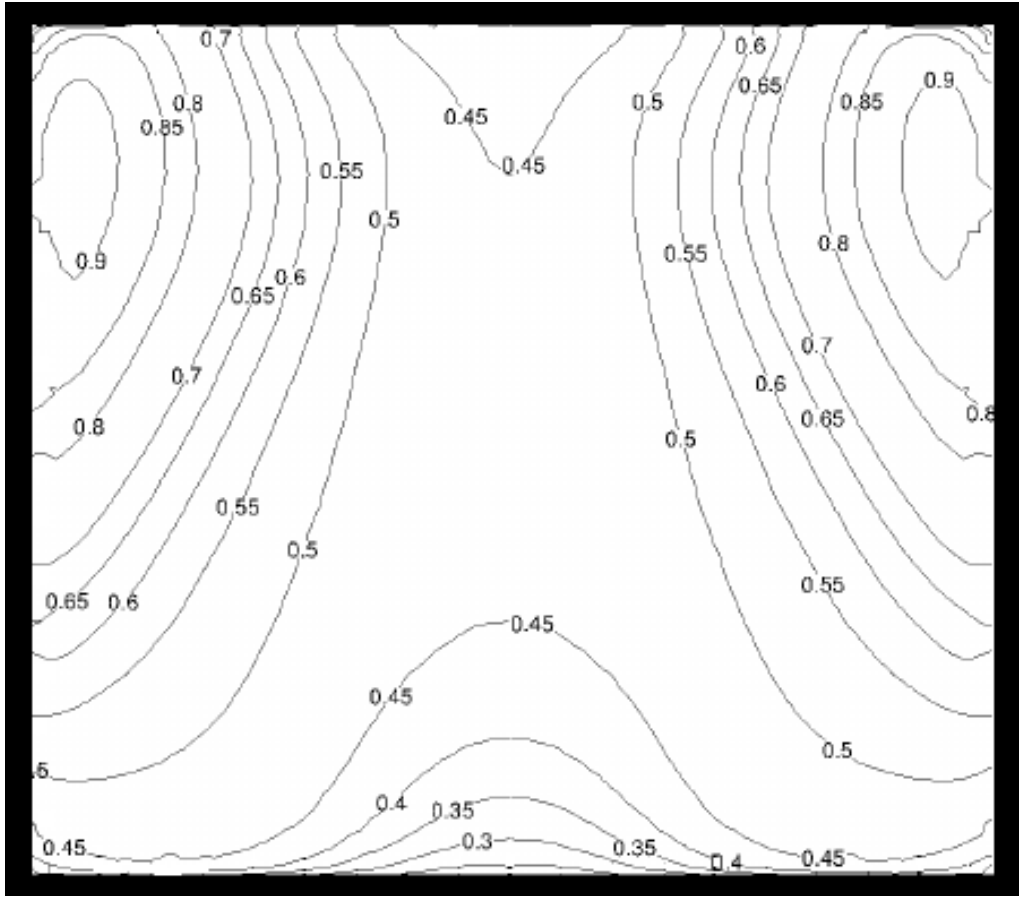

(a) Exchange Inlet

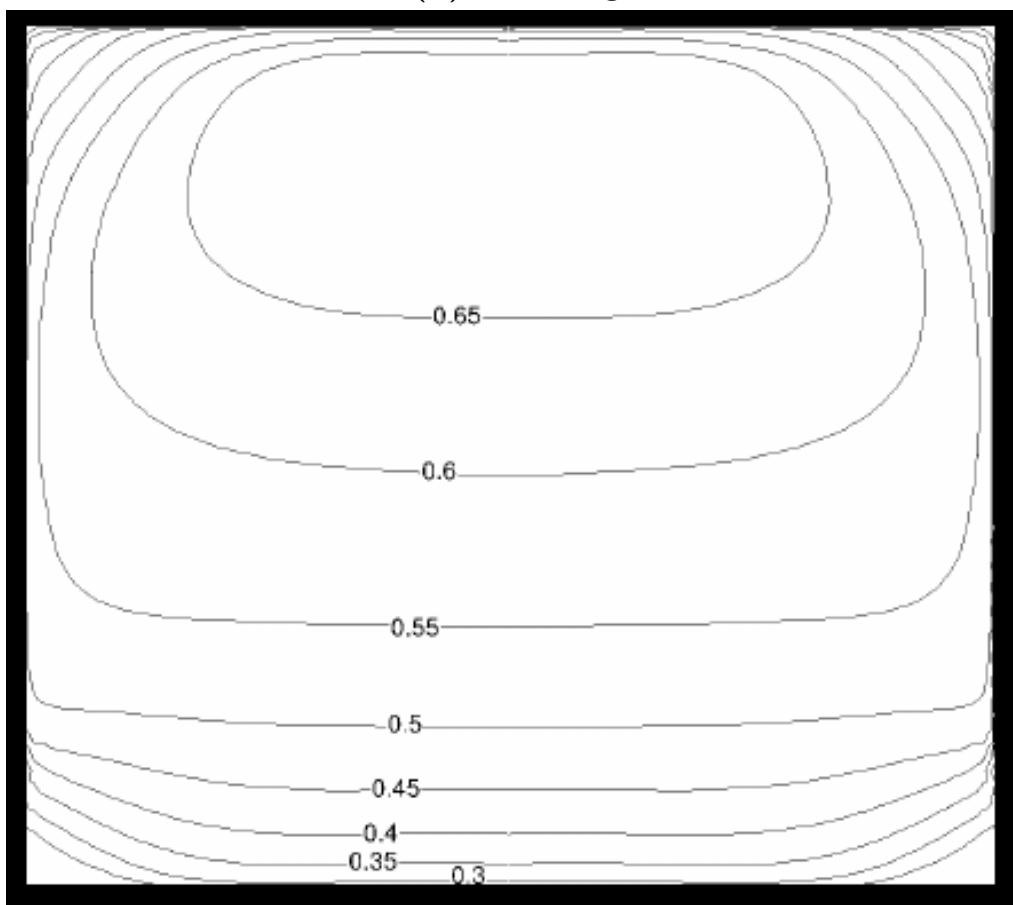

(b) Twin Circular

Figure 29: Low Rocket Chamber Pressure $\mathrm{O}_{2}$ Partial Density at EEP 


\section{Streamwise Planes}

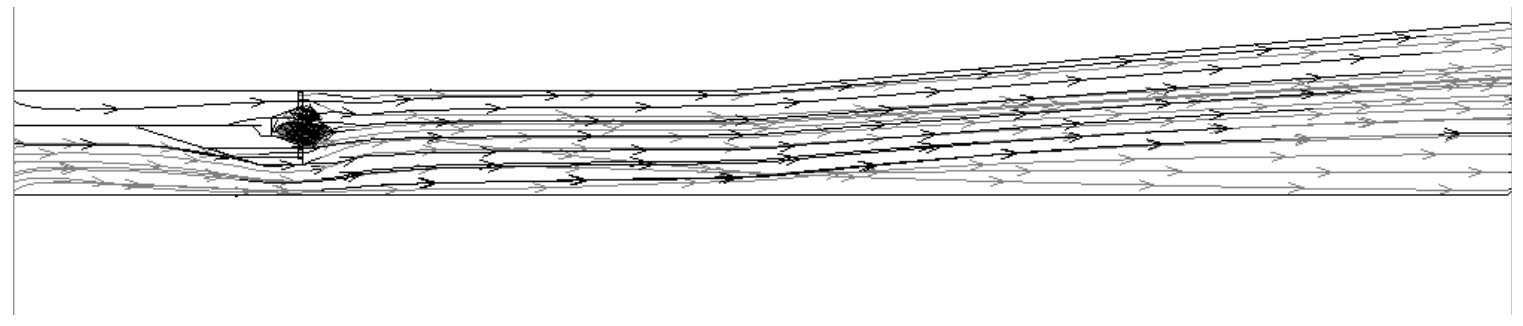

Figure 30: Stream Trace along Rocket Flow Plane of the Exchange Inlet $(28 \mathrm{~mm}$ offset of the symmetry plane)

Investigating the stream trace of the flow from Figure 30 of the Exchange Inlet shows that almost all of the flow is moving through the engine without recirculation. Some areas of interest are the entrance of the entrainment duct in which flow is forced to arc around the inlet. Another area of interest is the recirculation caused inside the cone by the nozzle which tends to aid mixing caused by the Exchange Inlet.

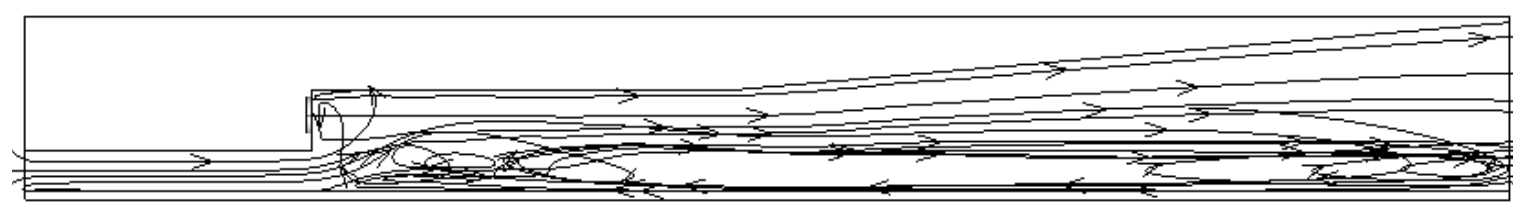

Figure 31: Twin Circular Low Pressure Stream Trace

In Figure 31 the streamtrace of the Twin circular configuration is shown. Despite having a thin plate at the inlet there is not a significant curve in the flow as it enters the entrainment duct. Near the RNEP, a recirculation area begins to form. This recirculation forces the rocket flow to compress and stay forced along the upper wall. The bulk of the Twin circular flow is outbound at the exit but there is some evidence of the recirculation. A recirculation is not desirable along the lower surface and reduces the amount of momentum at the outlet, reducing efficiency of the engine.

In Figure 32 a streamwise pressure plot centered on the rocket nozzle for the 


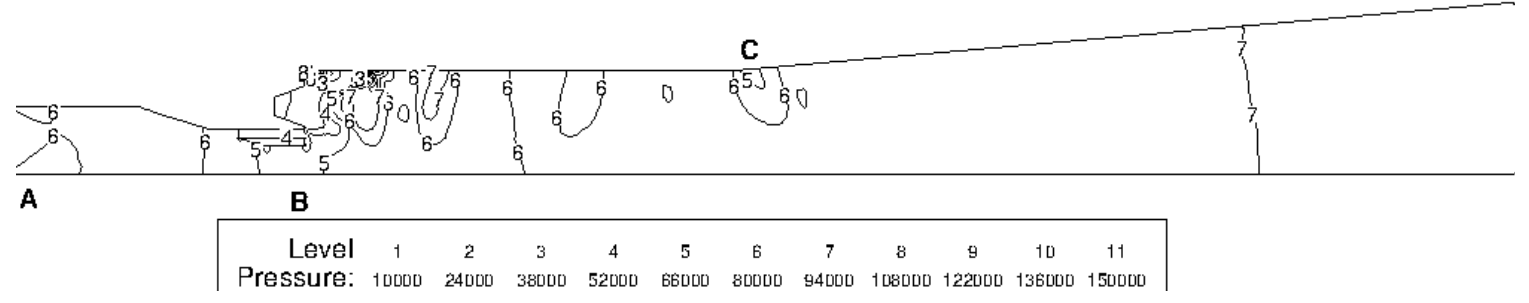

Figure 32: Exchange Inlet Rocket Flow Plane Pressure Plot

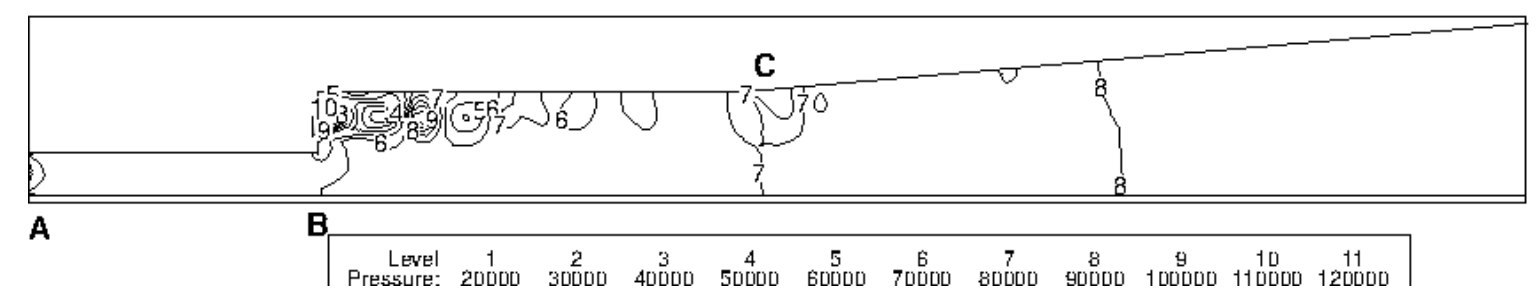

Figure 33: Twin Circular Rocket Flow Plane Pressure Plot

Exchange Inlet is shown. It is seen that the locations with strong oscillations in pressure indicate the existence of a shock train just after the RNEP above location B. At the location $\mathrm{C}$ where the expansion occurs there is a reduction in pressure as a result of the supersonic flow in this location.

The Twin circular pressure plot is shown in Figure 33. The shock train can be seen as rocket flow exits the rocket nozzle. The pressure values drop more significantly than in the Exchange Inlet. There is significant reflection of the shock between the flow and the upper wall. As the flow reaches the expansion duct at $\mathrm{C}$ there is a decrease in pressure as a result of the sonic flow similar to the behavior seen in the Exchange Inlet at this location.

The $\mathrm{O}_{2}$ partial density along the rocket flow plane shown in Figure 34 is for the Exchange Inlet. It shows that the flow from the nozzles mix well with the entrained air stream from the entrainment duct, increasing the average flow partial density of $\mathrm{O}_{2}$. The flow tends to concentrate along the upper surface and slowly dissipates after 


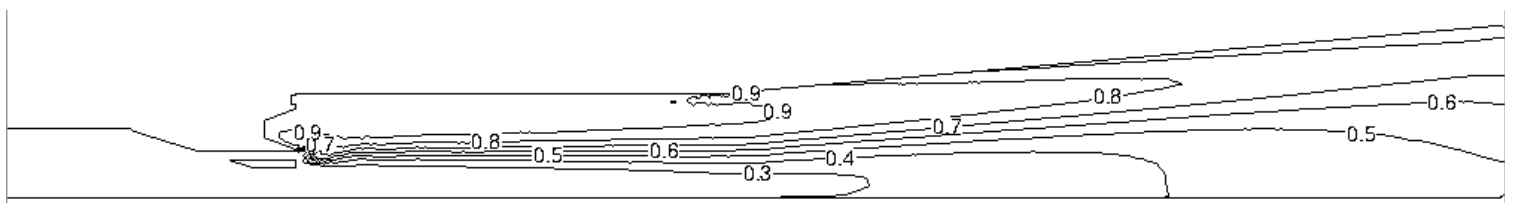

Figure 34: Exchange Inlet $O_{2}$ Mass Fraction along Rocket Flow Plane

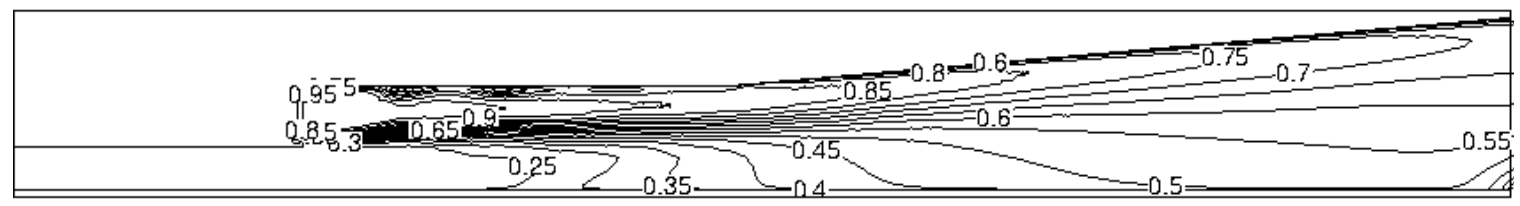

Figure 35: Twin Circular $\mathrm{O}_{2}$ Partial Density along Rocket flow Plane

the expansion duct begins.

In Figure 35 the $\mathrm{O}_{2}$ partial density for the Twin circular is shown. Due to the recirculation, the $\mathrm{O}_{2}$ partial density begins to increase before the halfway mark of the straight duct. The recirculation aids in the mixing process, however if this is not physically accurate, mixing would be less. The bulk of the high $\mathrm{O}_{2}$ partial density flow follows the upper surface.

Table 6 summarizes the results found in this section.

\begin{tabular}{|c|c|c|}
\hline Configuration & Twin circular & Exchange Inlet \\
\hline Mass Flow Air $\left(\dot{m}_{\text {air }}\right)$ & 0.172 & 0.258 \\
\hline Mass Flow Rocket $\left(\dot{m}_{r}\right)$ & 0.235 & 0.321 \\
\hline Entrainment Ratio & 0.7319 & 0.804 \\
\hline $\bar{M}$ Exit Plane & 0.300 & 0.415 \\
\hline $\bar{O}_{2}$ Exit Plane & $55.8 \%$ & $57.0 \%$ \\
\hline
\end{tabular}

Table 6: Summary Of Low Pressure configuration Results 


\subsubsection{High Pressure Cold Gas Configuration}

Similar to the low pressure cold gas cases, the high pressure Exchange Inlet and Twin circular configuration will be examined and compared. The pressures examined are $1415 \mathrm{kPa}$ for the Exchange Inlet and $1436 \mathrm{kPa}$ for the Twin circular configuration. High pressure test cases are considered as they represent more realistic rocket conditions as well as increasing the likelihood of a choked condition in the entrainment duct in both configurations.

\section{Wall Pressures}
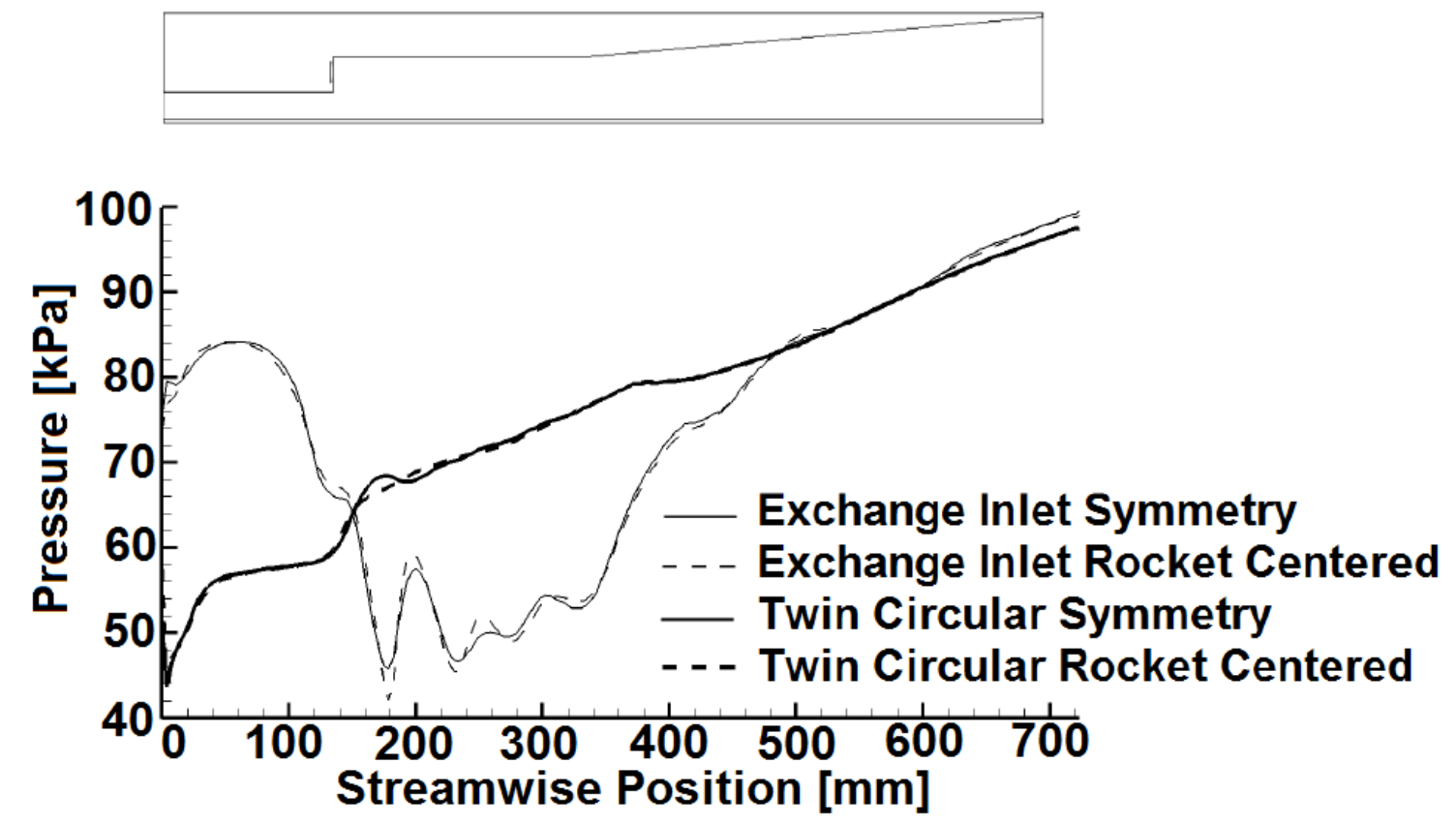

Figure 36: Lower Surface High Pressure Distribution for Long Engine Configuration

From Figure 36 it is seen that the minimum pressure of the Exchange Inlet configuration is at a location $175 \mathrm{~mm}$ downstream of the Engine Inlet. This indicates that a Fabri Choke is occurring as a result of the expansion of the supersonic flow. The pressure line then shows some oscillations along the lower surface as a result of shocks reflecting from the supersonic flow and the lower wall. A minimum pressure 
of $46.9 \mathrm{kPa}$ is seen from the Exchange Inlet configuration at the $180 \mathrm{~mm}$ location while the Twin circular configuration shows a minimum pressure at the inlet of 45 $\mathrm{kPa}$ that quickly rises to $55 \mathrm{kPa}$. The Twin circular configuration pressure rises to $58 \mathrm{kPa}$ where it is sustained until the RNEP where it rises to $68 \mathrm{kPa}$ and linearly expands to the pressure at the EEP of $101 \mathrm{kPa}$. The high pressure Twin circular configuration shows no Fabri choking. For both the Twin and Exchange configurations, the pressure distributions show similar values indicating uniform acceleration along the lower surface.

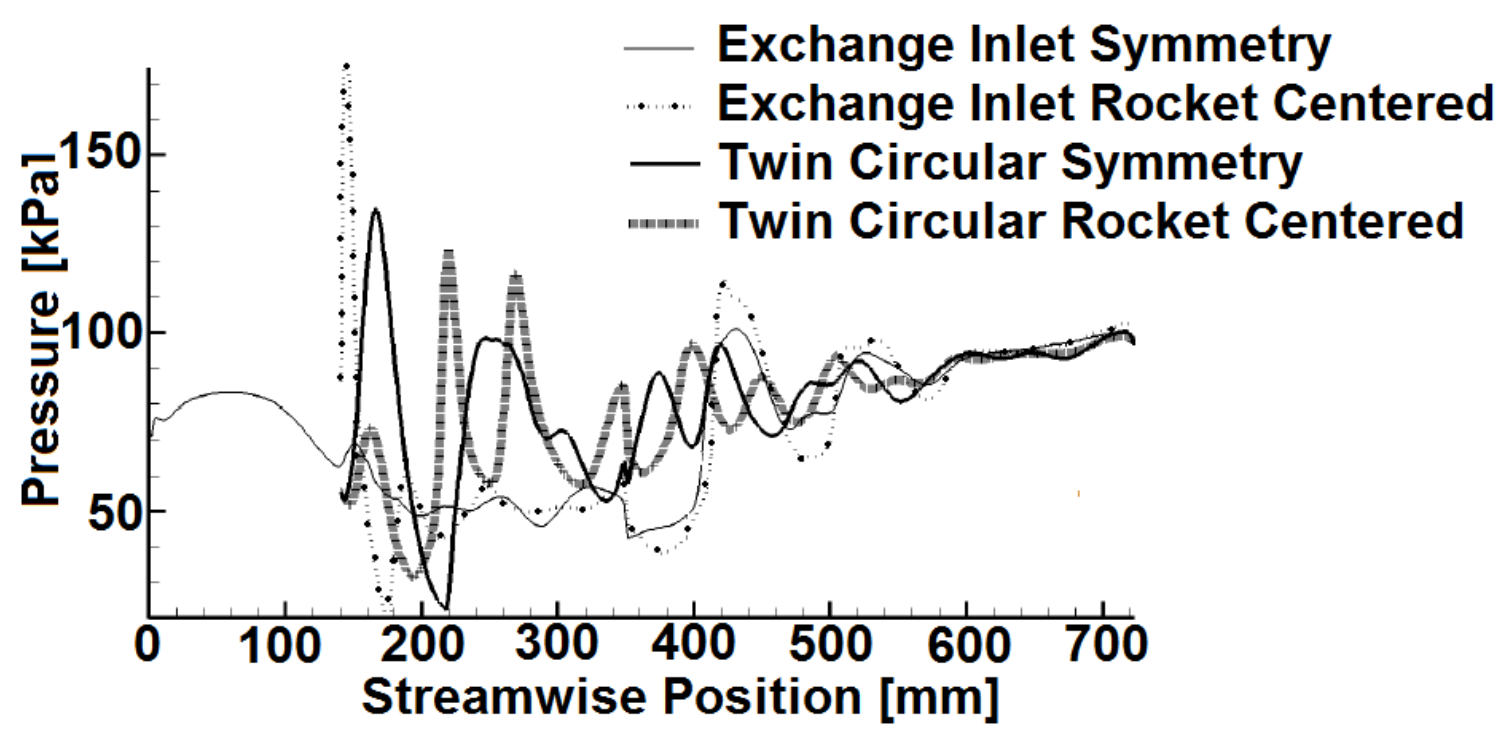

Figure 37: Upper Surface High Pressure Distribution for Long Engine configuration

Figure 37 shows the pressure distribution of the upper surface along the two streamwise planes (symmetry and $28 \mathrm{~mm}$ offset of the symmetry plane). The line following the symmetry plane for the Exchange Inlet reaches a minimum pressure of $50.1 \mathrm{kPa}$ and has some oscillations throughout the engine. Upon reaching the expanding section, the pressure rises up to atmospheric pressure and oscillates 3 more times before settling to atmospheric pressure. In contrast, the Twin configuration 
shows pressure oscillations along the symmetry line through to the exit. The streamwise line centered above the rocket nozzle for the Exchange Inlet shows a high peak pressure due to the shock train reflecting off the upper wall as a result of proximity to this surface. The pressure oscillations dampen shortly after the $200 \mathrm{~mm}$ location and begin to follow a similar trend to the symmetry line. The Twin circular configuration shows significant oscillation through to the exit along both planes indicating that the shock train does not dissipate as quickly in the Twin circular configuration as the Exchange Inlet.

\section{RNEP And ESP}

As with the low pressure case, the RNEP and ESP are examined.

Figure 38 (a) shows no flow from the entrained air area increasing to Mach 1 for the Exchange Inlet. The maximum Mach number is 0.82. Two of the entrainment tubes do not reach a choked condition, however, the lower entrainment tube reaches a choked condition. The reason for the slower flow at this plane than in the low pressure case is the under expanded flow out of the rocket nozzle which creates the Fabri choke just $35 \mathrm{~mm}$ downstream of this plane as was demonstrated by the low pressure location in Figure 36. The Exchange Inlet produces an entrained air mass flow of $0.244 \mathrm{~kg} / \mathrm{s}$ and an entrainment ratio of 0.409 . This is $5.4 \%$ less $m_{\text {air }}$ than at the low pressure $(0.258 \mathrm{~kg} / \mathrm{s})$ and a decrease of $49.1 \%$ in entrainment ratio.

Figure 38 (b) shows the RNEP of the Twin circular configuration and shows that the flow accelerates to Mach 0.918 near the rocket nozzles. The accelerated flow appears similar to that of the low pressure Twin case in that the peak location is near the symmetry plane and upper wall of the entrained air area. The flow pattern shows that the flow is more accelerated near the rocket nozzle flow as was done with the low pressure case. Though it appears that the flow is accelerated more in the Twin configuration, the entrainment tubes of the Exchange Inlet configuration help 


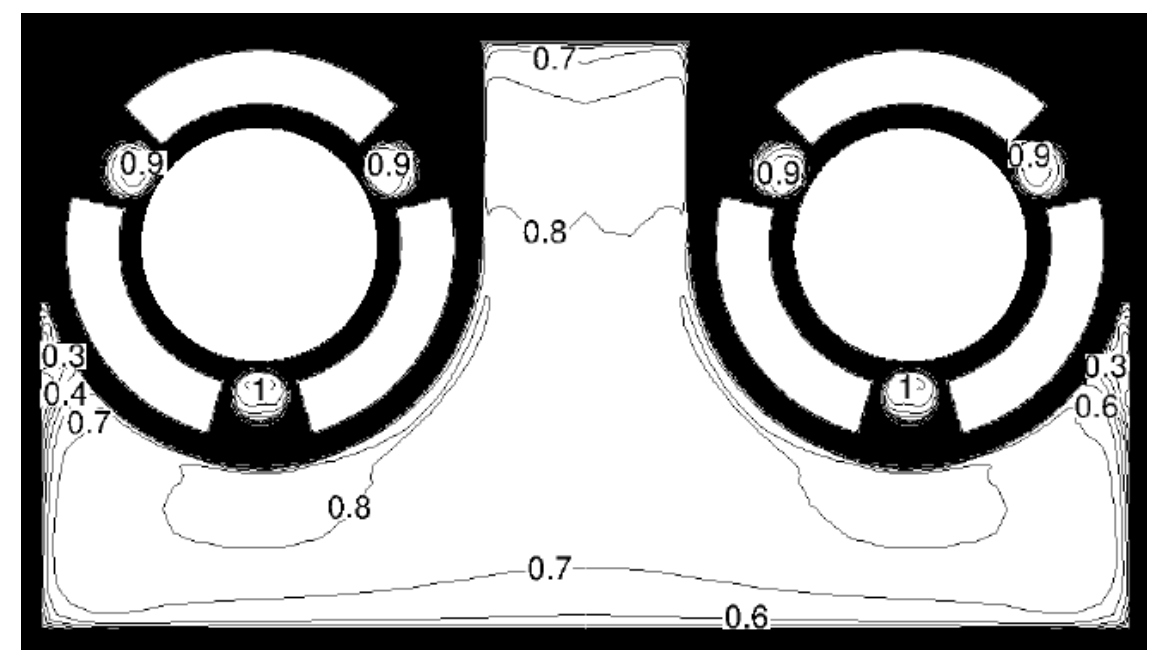

(a) Exchange Inlet

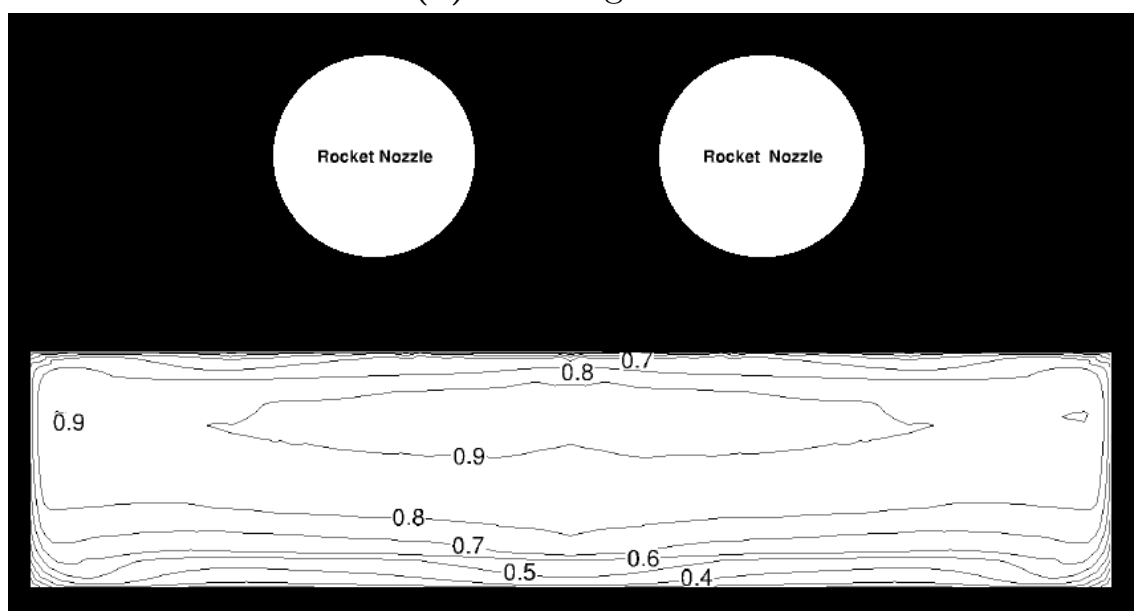

(b) Twin Circular

Figure 38: High Rocket Chamber Pressure Mach Contours at RNEP 


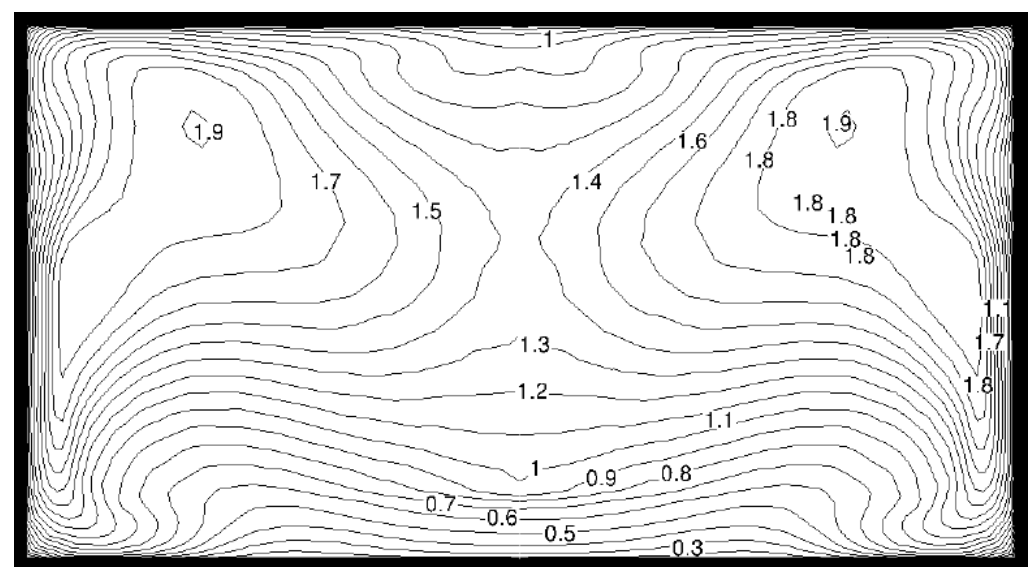

(a) Exchange Inlet

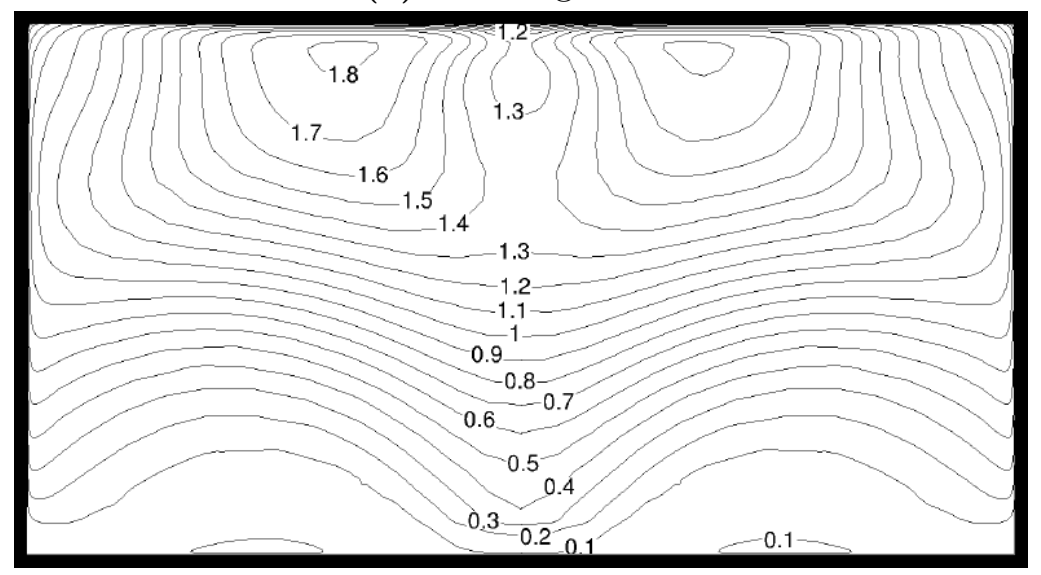

(b) Twin Circular

Figure 39: High Rocket Chamber Pressure Mach Distribution at ESP

to increase the air entrainment over the Twin circular configuration. Twin circular configuration has an entrainment ratio of 0.384 and an air mass flow rate of $0.186 \mathrm{~kg} / \mathrm{s}$. This represents an increase in $\dot{m}_{\text {air }}$ of $8.1 \%$ but a decrease of $47.5 \%$ in entrainment ratio compared to the low pressure case.

Figure 39 (a) shows the ESP of the Exchange Inlet configuration. As a result of the acceleration of the entrained air, this configuration sees higher Mach numbers throughout the plane as compared to the Twin configuration in Figure 39 (b). Similar to the low pressure configuration, the higher supersonic flow is focused along the outer wall of the engine. A significant portion of the flow is greater than Mach 1.0 . The 
Twin configuration has a more concentrated rocket core. Figure 39 (b) shows the ESP of the Twin circular configuration where the supersonic rocket core holds near the upper wall. The upper half of the flow has supersonic values in the Twin configuration while from the Exchange configuration over $3 / 4$ of the flow is supersonic.

\section{EEP}

At the outbound plane shown in Figure 40 (a) a similar trend to the lower pressure Exchange Inlet case from Figure 28 can be seen where the high speed flow dominates towards the upper outer corners. Along the centerline near the lower surface, there is a recirculation zone which creates the resulting region of low Mach number. The peak Mach number is 1.14 while the average Mach number is 0.703 which is an increase of $69.4 \%$ compared to the low pressure case.

Figure 40 (b) shows the Mach distribution at the outlet of the engine of the Twin circular configuration. A similar trend to the low pressure case for the Twin circular configuration (Figure 28 (b) with regards to the location of the peak Mach numbers. High Mach flow is seen along the symmetry plane and upper surface although now the two high speed zones have merged along the symmetry plane. In addition, there exists recirculation in the high pressure case at the outbound plane along the span of the lower wall with a minimum near the lower corners, an effect not seen in the low pressure case. The peak Mach number seen is 1.21 while the average Mach number is 0.681 , an increase of $127 \%$ in the average compared to the low pressure case.

The outbound $\mathrm{O}_{2}$ plane shown in Figure 41 (a) of the Exchange Inlet shows a strong tendency for the pure rocket flow to be maintained along the upper outer surface. A recirculation along the centerline causes the flow partial density to be lower along the centerline. A maximum partial density of $96.3 \%$ and a minimum of $23.2 \%$ is found for the Exchange Inlet configuration. A mean value of $64 \% \mathrm{O}_{2}$ partial density is found. 


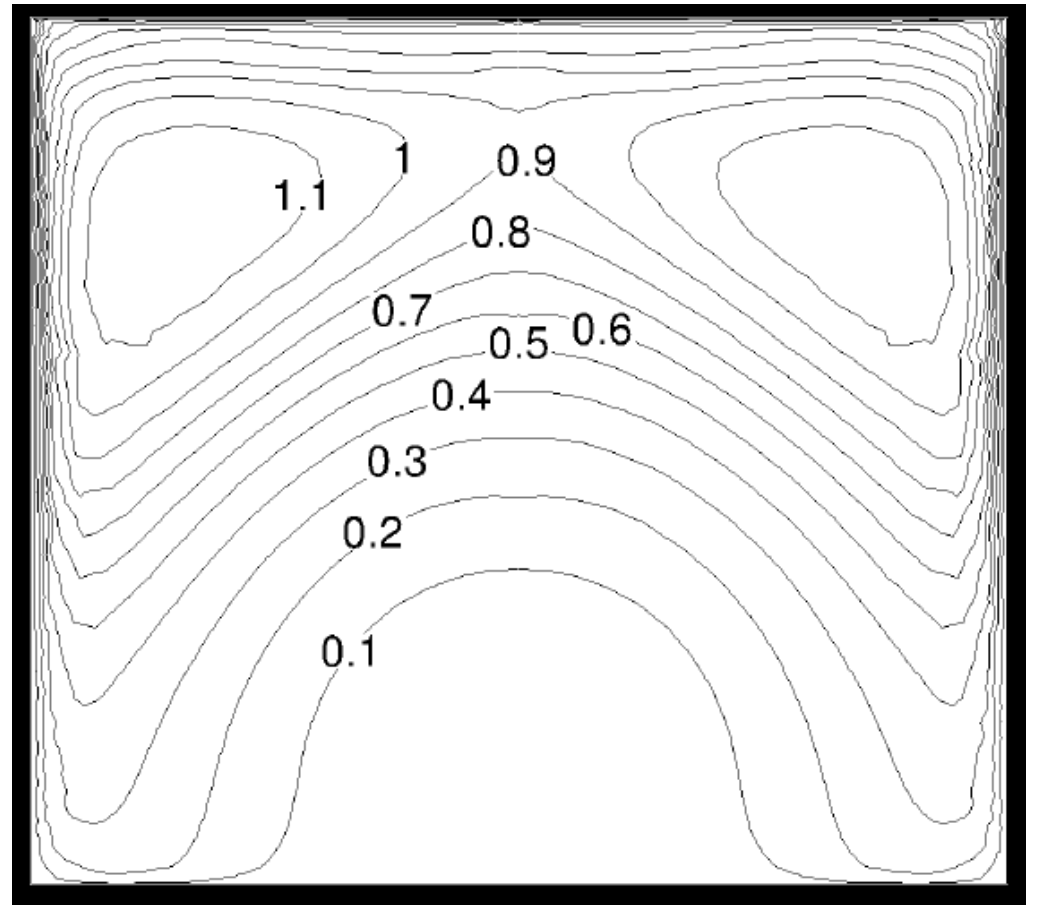

(a) Exchange Inlet

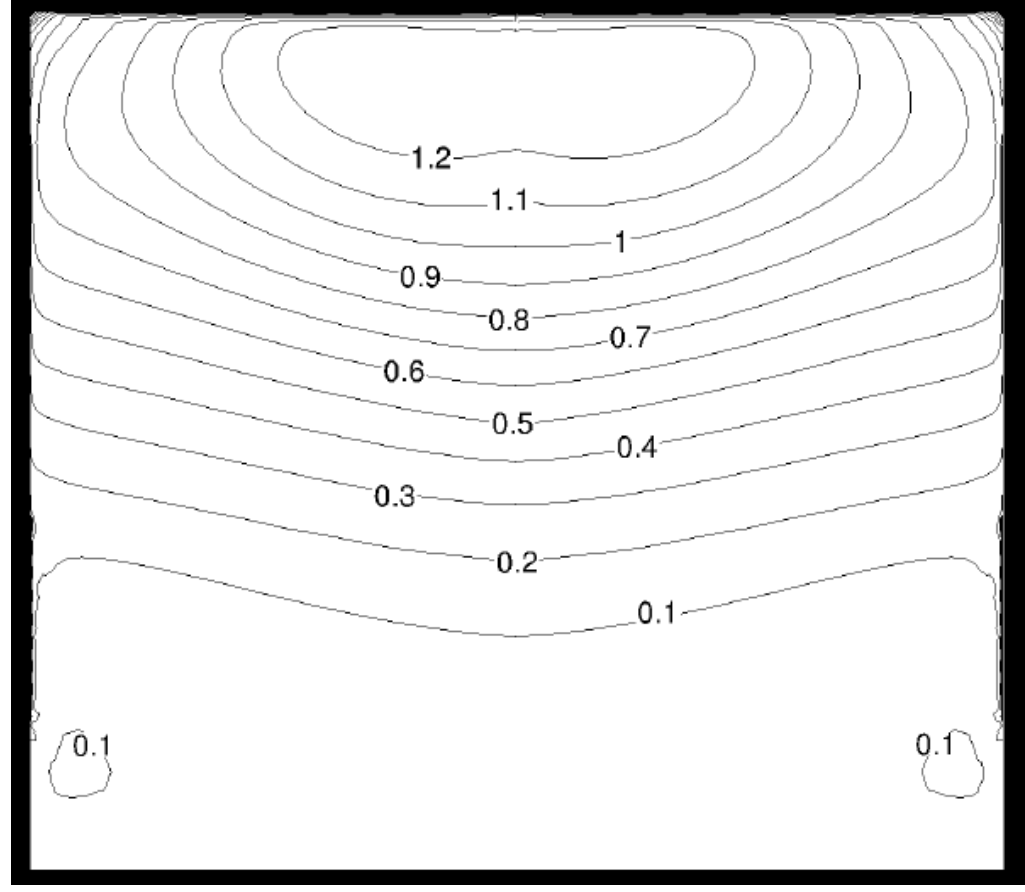

(b) Twin Circular

Figure 40: High Rocket Chamber Pressure Mach Distribution at EEP 


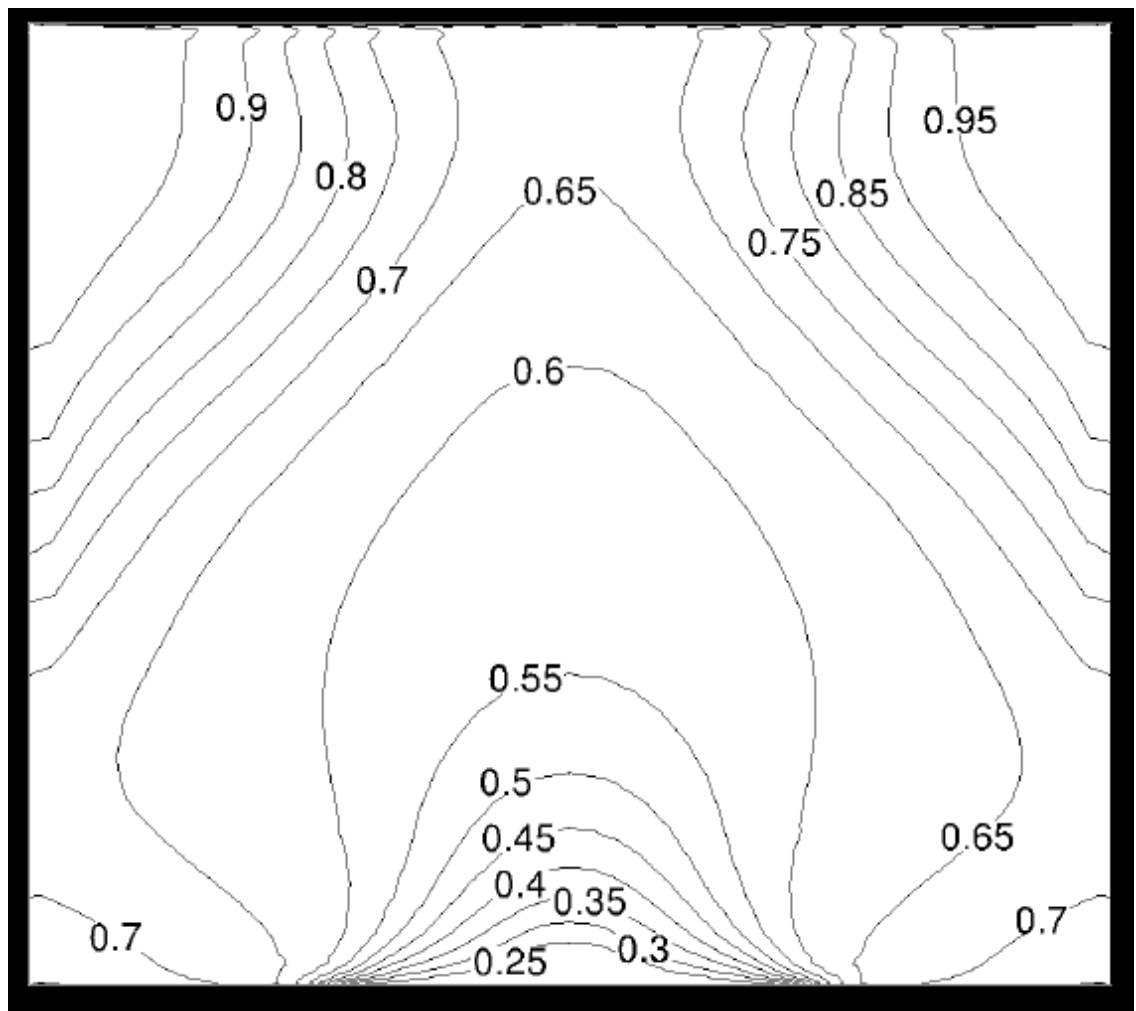

(a) Exchange Inlet

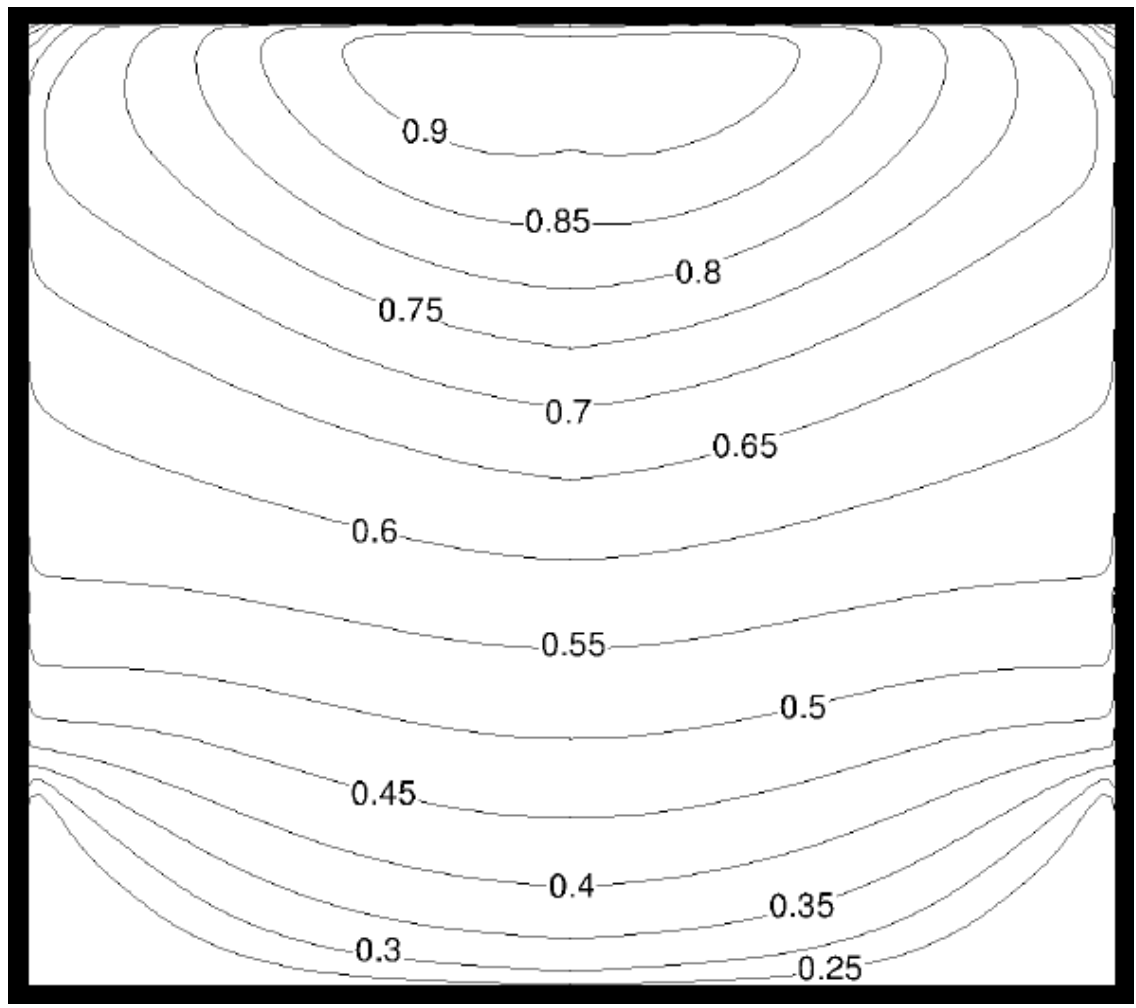

(b) Twin Circular

Figure 41: High Rocket Chamber Pressure $\mathrm{O}_{2}$ Partial Density at EEP 
The flow shown in Figure 41 (b) shows that the nearly pure $\mathrm{O}_{2}$ partial density is maintained along the upper wall near the symmetry plane for the Twin circular configuration. The $\mathrm{O}_{2}$ partial density is very similar to the lower pressure Twin circular configuration in trend but with higher peak values. The peak value of $\mathrm{O}_{2}$ partial density is $92.8 \%$ and the minimum value is $22 \%$ in the lower corners as a result of the recirculation. The two rocket cores produce a high partial density to be focused along the symmetry plane on the upper surface. An average $\mathrm{O}_{2}$ partial density of $62.8 \%$ is found on this plane.

\section{Streamwise Planes}

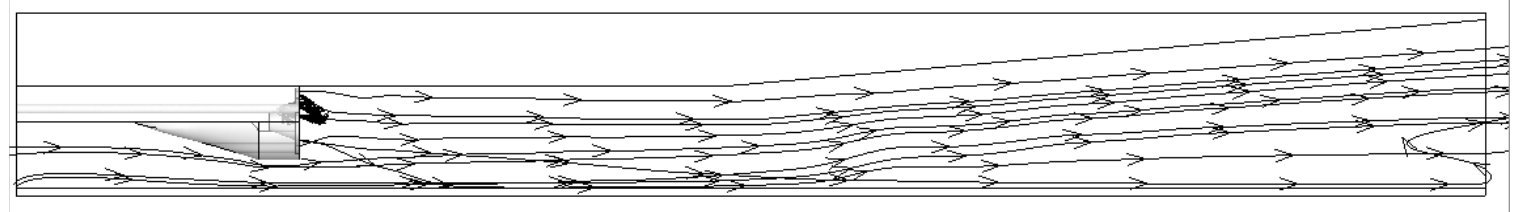

Figure 42: Exchange Inlet configuration Stream Trace High Pressure (28 mm offset of symmetry plane)

The stream trace in Figure 42 of the high pressure case Exchange Inlet displays the recirculation zone at the EEP as was mentioned in Figure 40 a . Like the lower pressure case, the flow at the entrainment duct inlet curves around the entrance to compresses through the narrow location below the rocket nozzle. There is again strong recirculation in the cone within the rocket nozzle. The recirculation at the outlet occurs near the symmetry plane.

In the stream trace shown in Figure 43 for the Twin circular configuration, recirculation is much more evident in the high pressure case. The flow separates further downstream in the high pressure case than in the low pressure case seen in Figure 31 , however, the recirculation area occupies more than $20 \%$ of the flow at the outbound 


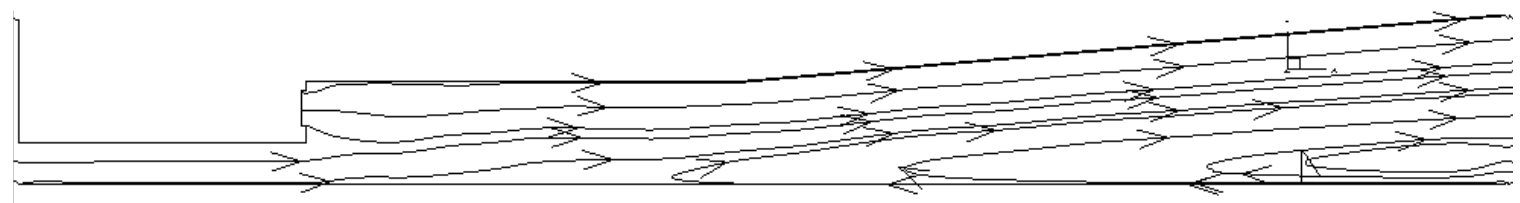

Figure 43: Twin circular High Pressure Stream trace

plane. The flow at the RNEP is under expanded and moves outwards as would be expected forcing some of the entrained flow to remain along the lower surface. However, the turbulence generated by the high velocity flow causes the separation to occur near the beginning of the expansion duct.

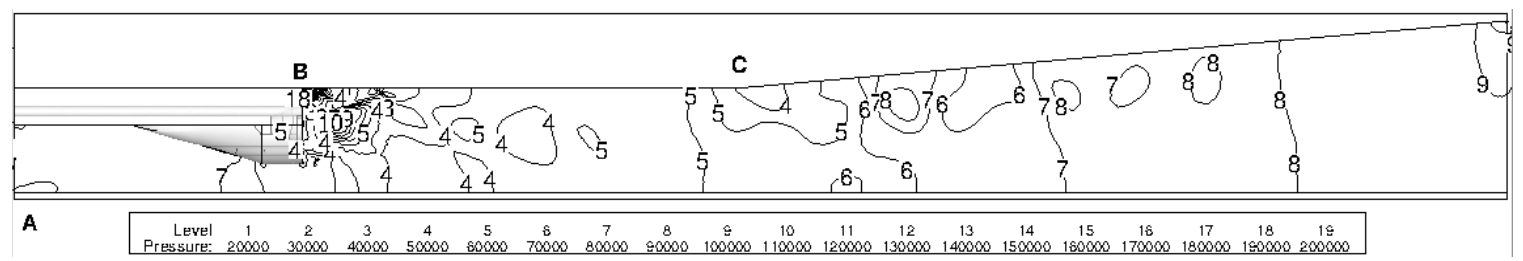

Figure 44: Exchange Inlet configuration Pressure Distribution

Figure 44 shows the pressure distribution inside the engine predicted for the Exchange Inlet. The data shows that at location A there is a slightly lower pressure as the entrained air moves around the corner and into the engine. At location $\mathrm{B}$ or the RNEP, there are significant regions of low and high pressure reflecting off the upper wall showing the existence of the shock train. After location B, there are some areas where the flow a significantly lower pressure condition. At the start of the expansion duct the shock train still persists and can be seen to continue almost completely to the outlet of the engine.

The pressure plot shown in Figure 45 shows the strong shock train that exists in the Twin circular configuration flow and the low pressure region reaching into entrainment duct. The entrained air expands almost immediately after passing the 


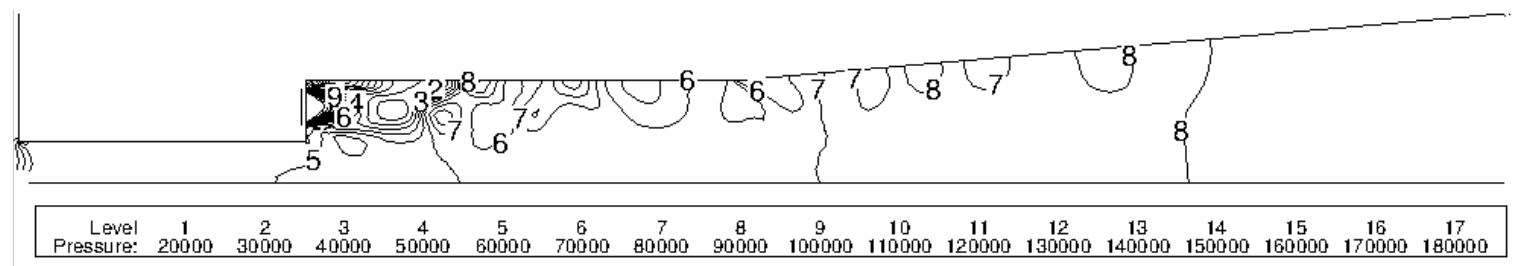

Figure 45: Twin circular configuration Engine Rocket Flow plane Pressure Plot

RNEP. Along the upper surface the shock train reflects off the upper surface multiple times similar to the Exchange Inlet. Around the corner of the expanding duct, the flow expands again as a result of the existing shock train. At the RNEP it is seen that the rocket nozzle flow does not quite expand into the entrained air which prevents the secondary choke that was seen in other flows.

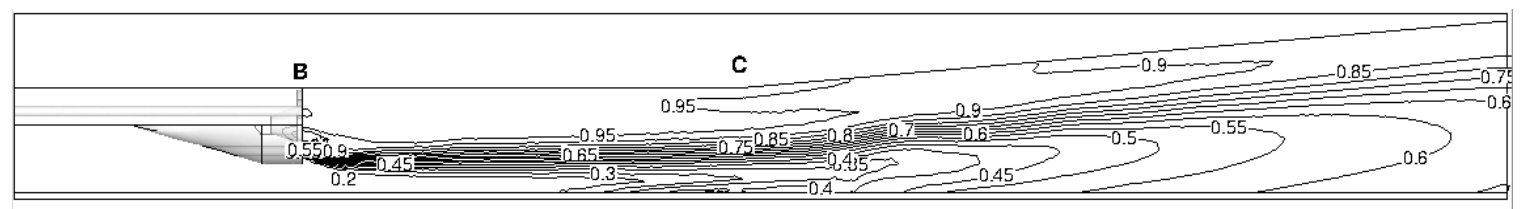

A

Figure 46: Exchange Inlet $\mathrm{O}_{2}$ Partial Density Rocket flow plane

The $\mathrm{O}_{2}$ partial density plots along the rocket flow plane of the Exchange Inlet are shown in Figure 46, It is seen that the $\mathrm{O}_{2}$ partial density begins to increase in a much shorter distance than with the lower pressure case. The regions of high $\mathrm{O}_{2}$ partial density are focused along the upper wall throughout the flow. At location $\mathrm{C}$ there begins to be more dissipation of the $\mathrm{O}_{2}$ partial density into the entrained air.

The $\mathrm{O}_{2}$ partial density plot shown for the Twin circular configuration in Figure 47 shows how the flow maintains a high partial density along the upper surface. The rocket flow mixes at a similar distance to the Exchange Inlet. The later onset of separation and recirculation is evident in the $\mathrm{O}_{2}$ plane as $\mathrm{O}_{2}$ partial density more gradually increases downstream. Due to the recirculation at the outlet, some flow 


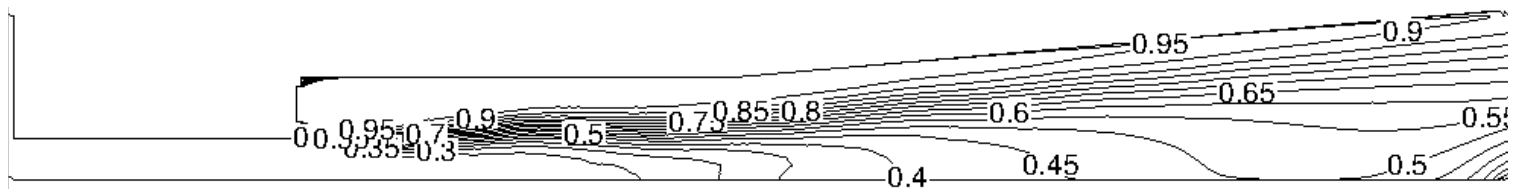

Figure 47: Twin circular configuration Engine Rocket Flow plane $\mathrm{O}_{2}$ Partial Density

\begin{tabular}{|c|c|c|c|c|}
\hline & \multicolumn{2}{|c|}{ High Pressure } & \multicolumn{2}{c|}{ Low Pressure } \\
\hline Configuration & Twin circular & Exchange Inlet & Twin circular & Exchange Inlet \\
\hline Mass Flow Air $\left(\dot{m}_{\text {air }}\right)$ & 0.186 & 0.2435 & 0.172 & 0.258 \\
\hline Mass Flow Rocket $\left(\dot{m}_{r}\right)$ & 0.485 & 0.596 & 0.235 & 0.321 \\
\hline Entrainment Ratio & 0.384 & 0.409 & 0.7319 & 0.804 \\
\hline $\bar{M}$ Exit Plane & 0.681 & 0.703 & 0.300 & 0.415 \\
\hline $\bar{O}_{2}$ Exit Plane & $64.1 \%$ & $62.8 \%$ & $55.8 \%$ & $57.0 \%$ \\
\hline
\end{tabular}

Table 7: Summary Of High Pressure Configuration Results

from outside the engine enters the engine and pushes the higher concentrated $\mathrm{O}_{2}$ flow upwards reducing the average partial density near the lower surface at the outlet. The high partial density of $\mathrm{O}_{2}$ is maintained well beyond the expanding duct beginning along the upper surface.

A summary of the key values is given in Table 7 . 


\subsection{Half Engine Configuration}

In the half engine configuration, the expanding duct (from $\mathrm{x}=350 \mathrm{~mm}(\mathrm{CC})$ to $\mathrm{x}=725 \mathrm{~mm}$ (DD) in Figure 10 is removed from the original engine design. The goal of this design is to investigate the mixing potential of the engine by reducing the engine length available for the mixing. In addition, a duct with an expanding section leading to atmospheric pressure creates a lower pressure region without the duct which can affect entrainment of air independent of the nozzle configuration. Therefore removing the expanding section results in any differences observed to be more directly attributable to the nozzle design itself.

\subsubsection{Low Pressure Cold Gas}

The low pressure cases will be at pressures of $740 \mathrm{kPa}$ and $783 \mathrm{kPa}$ for the Twin circular and Exchange Inlet configurations respectively. These two configurations are compared to each other and the entrainment properties discussed.

\section{Wall Pressure}

Figure 48 shows the pressure trends along the lower surface of the Exchange Inlet and Twin circular Short configurations. The Exchange Inlet has a minimum pressure of $85 \mathrm{kPa}$ at the RNEP while the Twin circular has a minimum pressure of $88 \mathrm{kPa}$ at the RNEP. At the duct inlet the Twin circular configuration reaches a lower pressure of $80 \mathrm{kPa}$. The Exchange Inlet shows a similar trend in the short configuration as is seen in the long configuration where the pressure decreases towards the RNEP and then increases as the flow expands into the straight duct. The Twin circular has a very flat trend in that from $50 \mathrm{~mm}$ to $300 \mathrm{~mm}$ the pressure remains between $87 \mathrm{kPa}$ and 90 $\mathrm{kPa}$. The lower pressure seen in the Exchange Inlet configuration indicates a higher Mach flow exists at the RNEP in the Exchange Inlet. The difference between the 


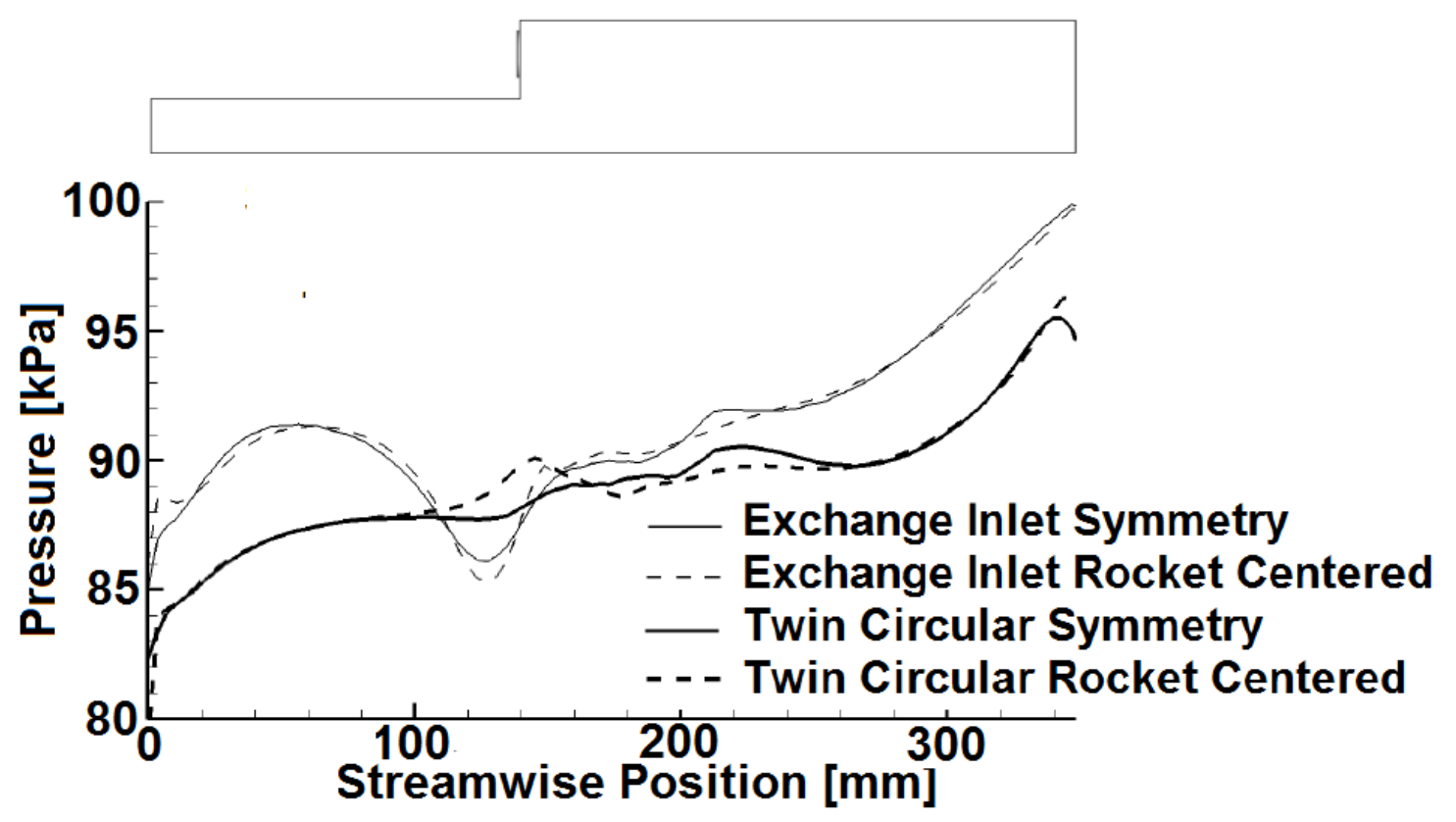

Figure 48: Lower Surface Low Pressure Distribution for Short Engine configuration

two nozzle configurations is far less pronounced in the short configuration than it is in the long configuration. The pressure distributions are close between the symmetry plane and the streamwise rocket centered plane for the Exchange Inlet but not for the Twin Circular showing a more uniform flow in the Exchange Inlet configuration.

Figure 49 shows the pressure along the upper surface. The symmetry line along the upper surface for the Exchange Inlet shows again the same trend as the long configuration at the lower pressure where a decrease in pressure occurs until the RNEP and then expansion occurs towards the exit thereafter. The streamwise line centered above the rocket for the Exchange Inlet shows oscillations occurring as a result of the shock train reflecting off the upper wall with the shocks quickly dissipating before the exit is reached. In comparison, the Twin circular configuration shows oscillations in pressure right until the EEP but of smaller overall magnitude. The line along the symmetry plane for the Twin circular configuration shows a low set of pressures that at the $150 \mathrm{~mm}$ location begin to expand to atmospheric pressure while the streamwise 


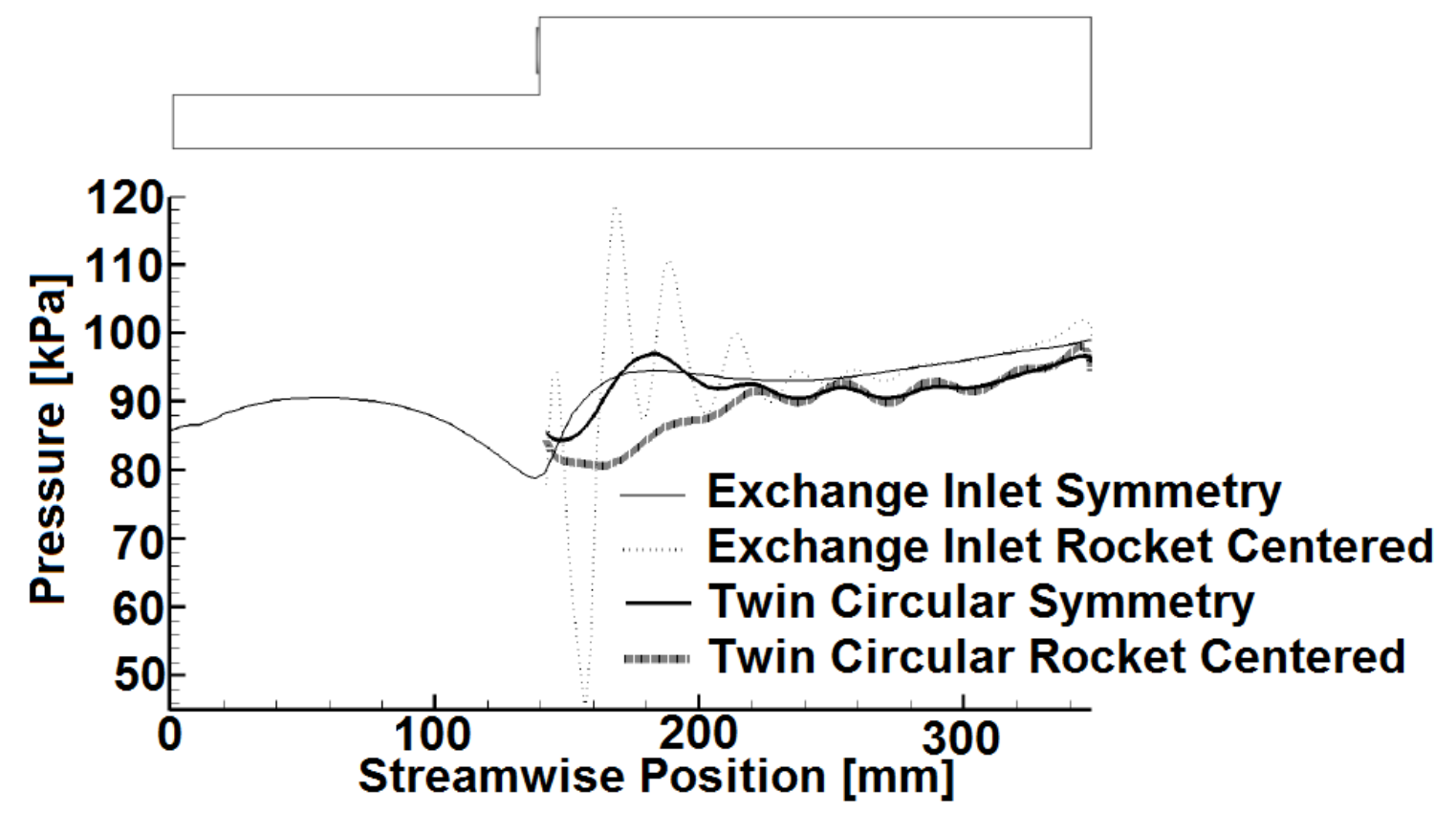

Figure 49: Upper Surface low Pressure Distribution for short Engine configuration

rocket centered line shows accelerated flow that has lower pressure from 175 to 250 $\mathrm{mm}$ than the symmetry plane but then expands towards the exit similarly to the symmetry plane.

\section{RNEP}

The RNEP is shown in Figure 50 (a) for the Exchange Inlet configuration. There are no regions where the flow accelerates to Mach 1 including the entrainment tubes. The maximum Mach number in the entrained duct is 0.61 which is significantly lower than the Exchange Inlet long configuration in Figure 26 (a) $(\mathrm{M}=1.1)$. In the entrainment tubes, the flow reaches a maximum Mach number of 0.7. The mass flow of the air through the RNEP is $0.250 \mathrm{~kg} / \mathrm{s}(3.2 \%$ less than in the long configuration) giving an entrainment ratio of 0.602 for the Exchange Inlet which is $25.1 \%$ lower than the long configuration.

The RNEP shown in Figure 50 (b) is the RNEP of the Twin configuration. It 


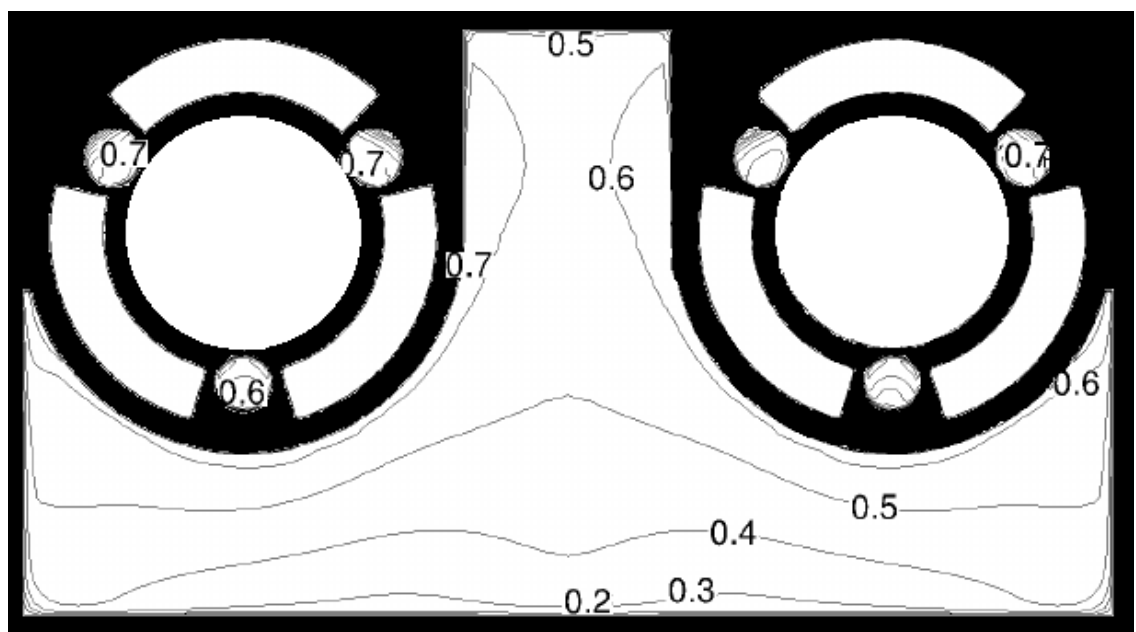

(a) Exchange Inlet

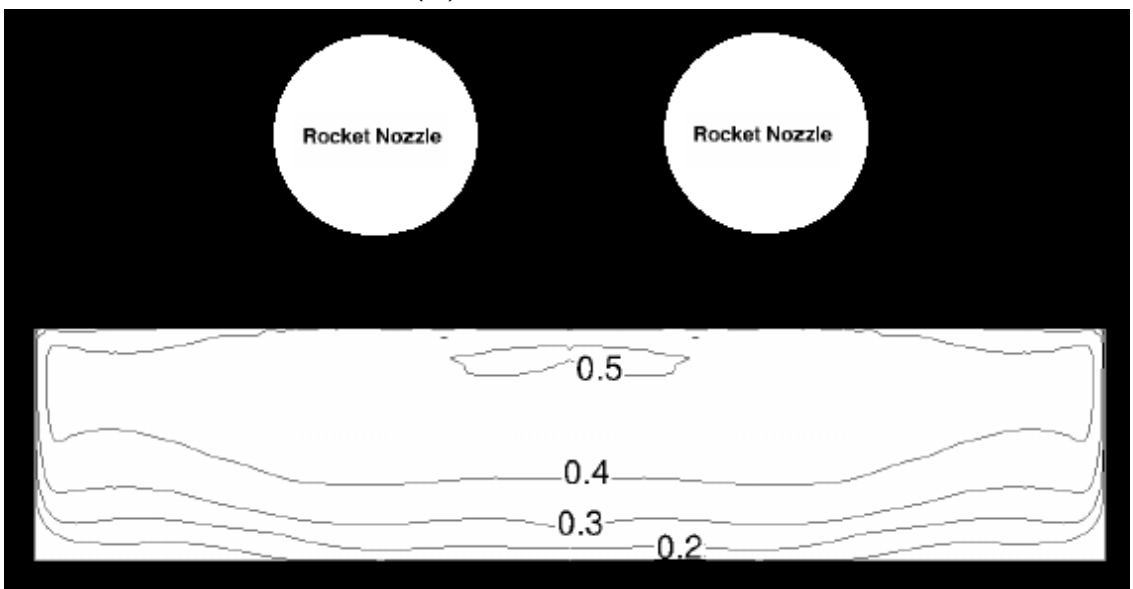

(b) Twin Circular

Figure 50: Short Configuration Low Rocket Chamber Pressure Mach Contours at RNEP 
shows a similar trend to the long configuration where the flow is more accelerated near the rocket nozzle. The flow however does not reach near the same peak values to the long configuration. The peak value in this plane is Mach 0.508. The entrained air mass flow is $0.122 \mathrm{~kg} / \mathrm{s}$ and the entrainment ratio is 0.445 for the Twin circular configuration as compared to $0.172 \mathrm{~kg} / \mathrm{s}$ and 0.7319 respectively for this rocket configuration with the longer duct. However comparing both the Twin circular and Exchange Inlet configurations without an expanding section, the Exchange Inlet shows higher peak Mach numbers as well as average values in the short configuration where $\dot{m}_{a i r} / \dot{m}_{r}$ is $18.9 \%$ greater in the Exchange Inlet

\section{EEP}

For the short engine configurations, since the expanding section is removed, the ESP is replaced by the EEP.

The outbound flow of the Exchange Inlet low pressure short engine is shown in Figure 51 (a). The peak value is Mach 1.14. The average value is 0.52. The lower surface does not accelerate significantly. The rocket core can still be seen in the EEP. This indicates a lower degree of mixing than compared to the long configuration as shown in Figure 28 (a).

The Mach distribution at the exit of the Twin circular short engine shown in Figure 51 (b) sees a more predominant rocket core when compared to both the Exchange Inlet short configuration 51 (a) and Twin circular long configuration 28 (b). The peak value is 1.2. However the numerical data predicts recirculation at the outlet plane. This causes the average Mach number to be lower. The average Mach number is 0.445 . The simulation predicts a large region of recirculated flow that is not as predominant in the long configuration. Though some separation occurs in the long configuration, near the outlet the long configuration only has a very small region of recirculation. 


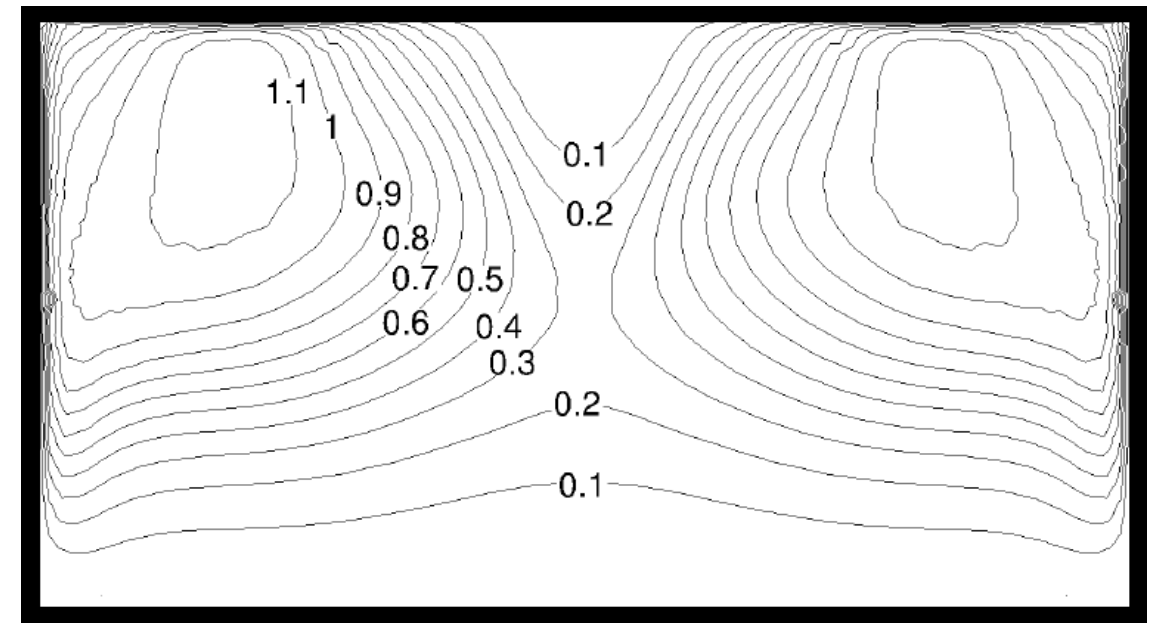

(a) Exchange Inlet

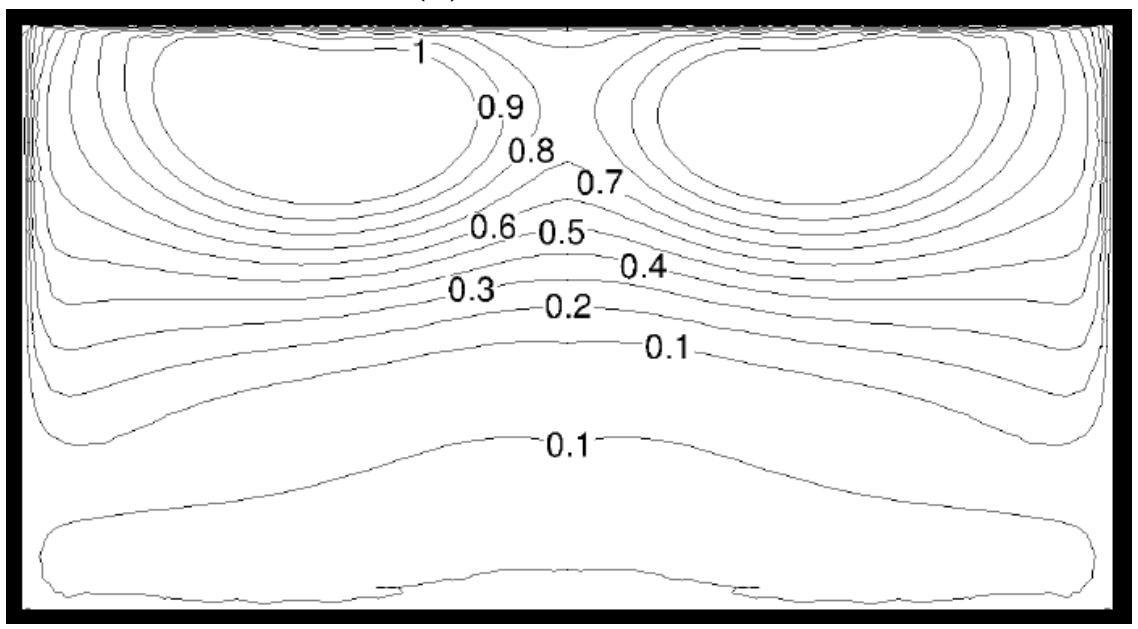

(b) Twin Circular

Figure 51: Short Configuration Low Rocket Chamber Pressure Mach Distribution at EEP 


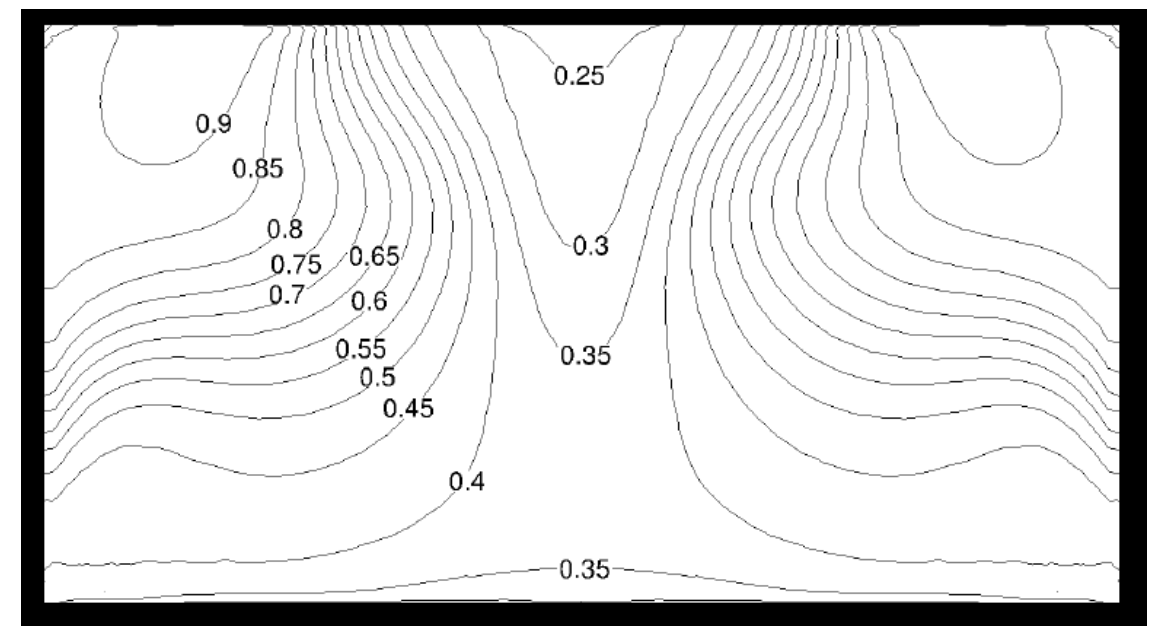

(a) Exchange Inlet

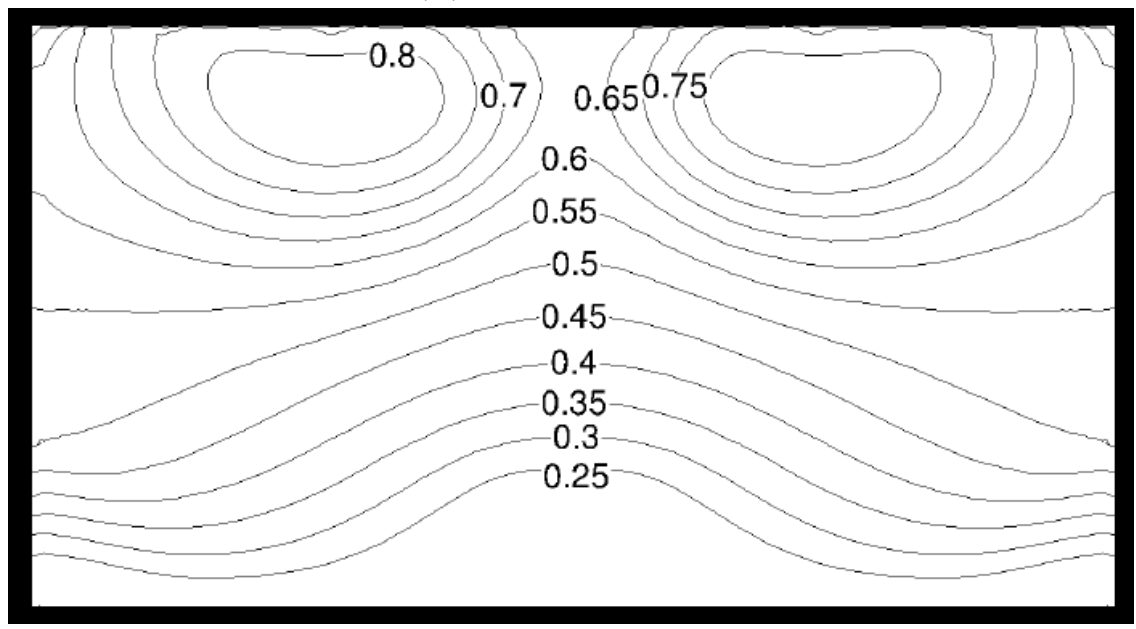

(b) Twin Circular

Figure 52: Short Configuration Low Rocket Chamber Pressure $\mathrm{O}_{2}$ Partial Density at EEP 
For the Exchange Inlet configuration $\mathrm{O}_{2}$ partial density plot at the EEP, Figure 52 (a) shows similar trends to the Mach distribution plane of Figure 51 (a) where flow is concentrated along the outer wall. The peak value is $92.6 \%$. The average $\mathrm{O}_{2}$ partial density is $55.7 \%$. Compared to the long configuration the peak values are nearly identical while the average values are $2 \%$ greater in the long configuration.

The $\mathrm{O}_{2}$ partial density shown in Figure 52 (b) for the Twin circular Short Engine configuration shows an unexpected pattern when considering Figure 51 (b) as $O_{2}$ is much more evenly distributed near the upper surface. The un-even distribution of high speed flow would make one assume that the partial density follows a similar trend. However, the process of diffusion of $\mathrm{O}_{2}$ is different than the transfer of momentum between the pure $\mathrm{O}_{2}$ rocket and entrained air. The peak value of $\mathrm{O}_{2}$ is $83 \%$. The location of the peak $\mathrm{O}_{2}$ partial density is in line with the rocket location. Both short configurations also suffer a recirculation on the lower surface which explains the lower $\mathrm{O}_{2}$ partial density along the lower surface. The minimum $\mathrm{O}_{2}$ partial density is $21.3 \%$, very close to the partial density of the ambient air. This indicates that the recirculation minimizes the partial density on the lower surface. The average $\mathrm{O}_{2}$ partial density is $51.5 \%$

\section{Streamwise Planes}

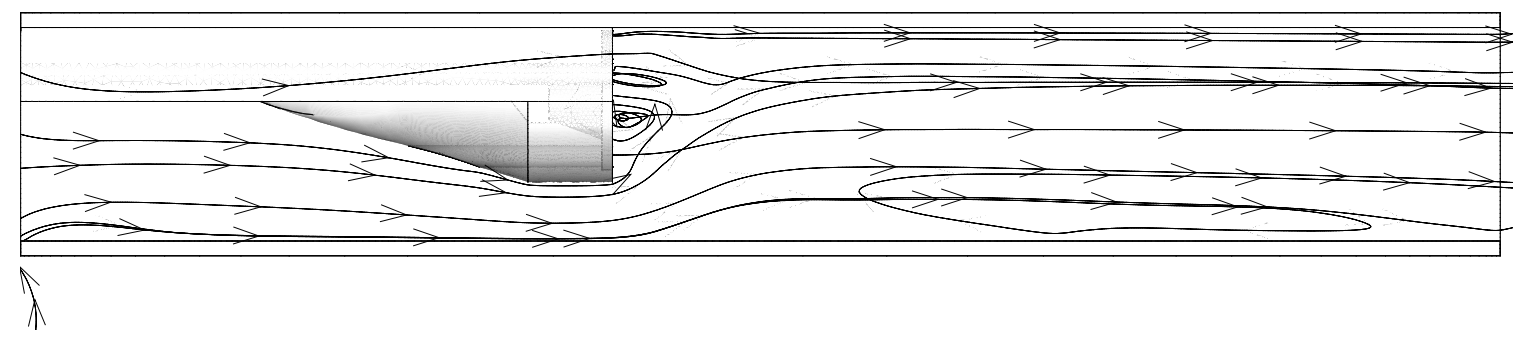

Figure 53: Exchange Inlet Short Engine Configuration Stream Trace

While the outbound plane shows no recirculation in the Exchange Inlet, the stream 
trace shown in Figure 53 shows that within the engine there is a small area of recirculation. There is some separation near the RNEP and some recirculation in this region, however the flow tends to re-attached before the EEP. The cone within the nozzle shows again recirculation as it did in the long configuration.

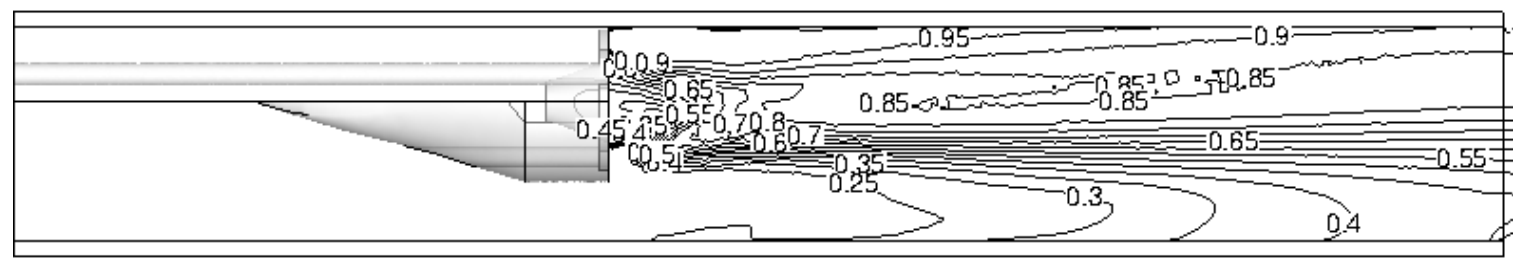

Figure 54: Exchange Inlet short engine $O_{2}$ Partial Density Rocket flow plane

Figure 54 shows the $\mathrm{O}_{2}$ partial density within the Exchange Inlet short configuration at the low pressure. Before the halfway location of the straight duct is even reached, the partial density reaches $30 \%$. A lower partial density of $\mathrm{O}_{2}$ near the upper surface of the flow implies that more entrained air is mixing with the rocket flow. The mixing occurs rapidly in the short engine which is not seen in the long configuration.

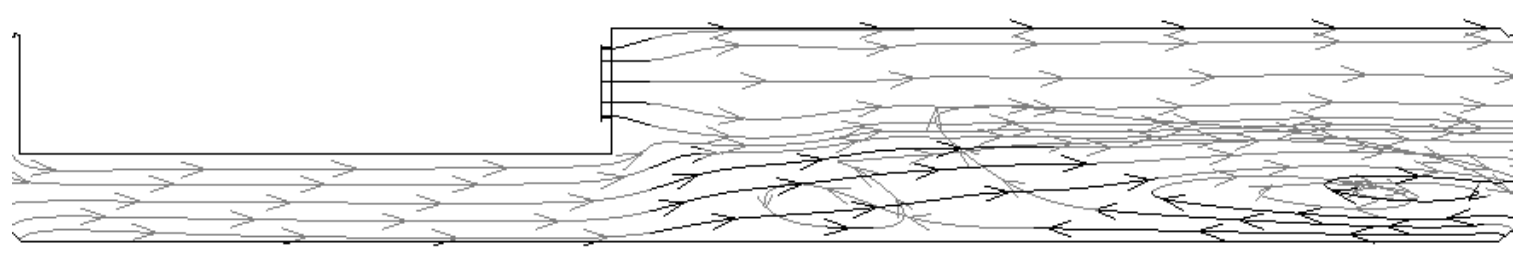

Figure 55: Twin circular Short Configuration Low Pressure Rocket Centered Stream Trace

Figure 55 shows a stream trace following the rocket centered streamwise plane. It is seen that within the short Twin circular configuration there is some recirculation near the lower surface from the EEP. 


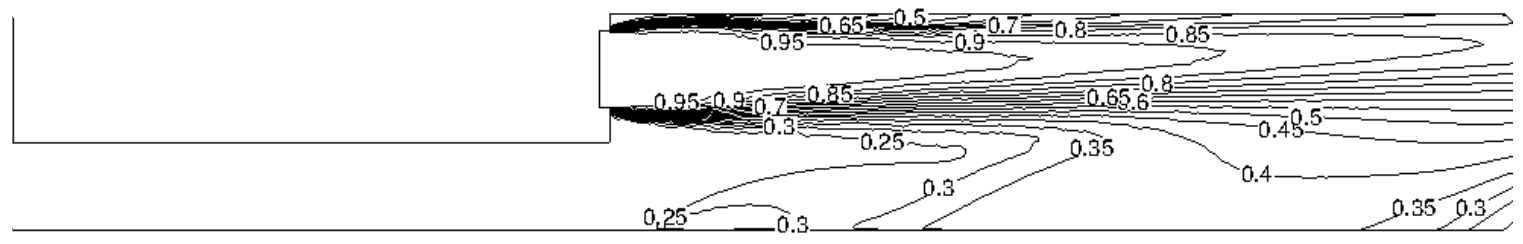

Figure 56: Twin circular Short Engine Rocket Flow Plane $O_{2}$ Partial Density

The rocket flow plane shown in Figure 56 shows the $O_{2}$ partial density along this plane for the Twin circular configuration. Here it is evident that some recirculation is occurring immediately after the rocket nozzle plane noted by the increase in $\mathrm{O}_{2}$ partial density in this location. The rocket flow does mix well with the entrained air although the recirculation appears to aid in mixing in a non-desirable way. The $\mathrm{O}_{2}$ dissipates quickly after the RNEP and is focused along the upper surface.

\begin{tabular}{|c|c|c|c|c|}
\hline & \multicolumn{2}{|c|}{ Short Configuration } & \multicolumn{2}{c|}{ Long Configuration } \\
\hline Configuration & Twin circular & Exchange Inlet & Twin circular & Exchange Inlet \\
\hline Mass Flow Air $\left(\dot{m}_{\text {air }}\right)$ & 0.122 & 0.198 & 0.172 & 0.258 \\
\hline Mass Flow Rocket $\left(\dot{m}_{r}\right)$ & 0.250 & 0.329 & 0.235 & 0.321 \\
\hline Entrainment Ratio & 0.488 & 0.602 & 0.7319 & 0.804 \\
\hline $\bar{M}$ Exit Plane & 0.445 & 0.520 & 0.300 & 0.415 \\
\hline $\bar{O}_{2}$ Exit Plane & $51.5 \%$ & $55.7 \%$ & $55.8 \%$ & $57.0 \%$ \\
\hline
\end{tabular}

Table 8: Summary Of Low Pressure Short and Long Configuration Results

A summary of the entrainment and mixing properties are given in Table 8 , The short configuration shows an increased entrainment ratio of the Exchange Inlet over the Twin circular configuration despite not having the expanding section which helps the entrainment process. This would indicate that despite engine length, the Exchange Inlet nozzle is more desirable over a circular nozzle. 


\subsubsection{High Pressure Cold Gas}

The high pressure configuration for both the Twin and Exchange Inlet configurations are also examined. The pressure values used are $1391 \mathrm{kPa}$ and $1430 \mathrm{kPa}$. The high pressure case is examined in order to investigate a realistic rocket chamber pressure. Additionally with the short engine configuration, the rocket flow may not expand completely to atmosphere by the EEP.

\section{Wall Pressures}

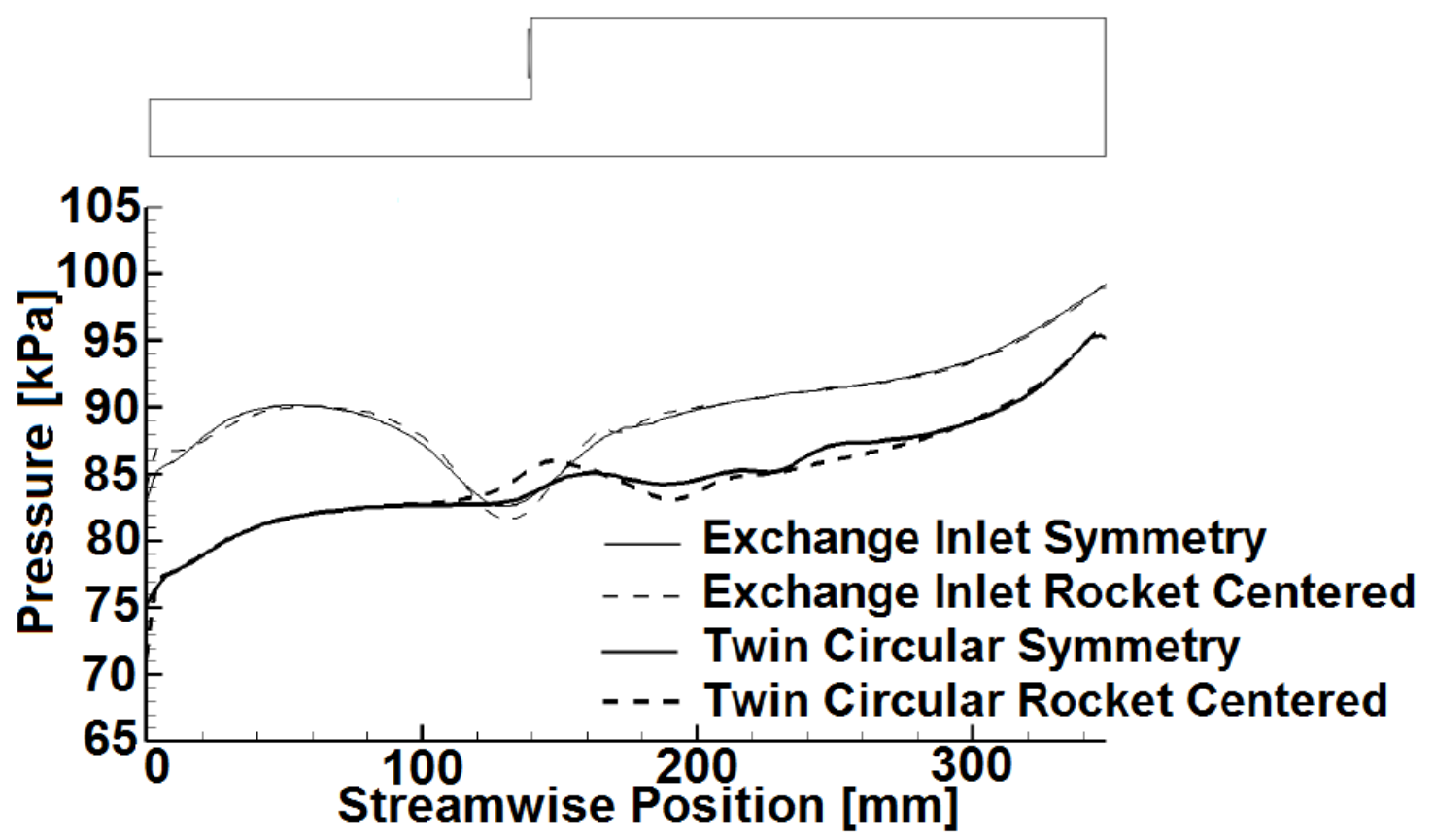

Figure 57: Lower Surface High Pressure Distribution for Short Engine configuration

The high rocket chamber pressure short engine configuration pressure data is shown in Figure 57. In the high pressure cases, the minimum pressure reached is nearly identical between both configurations. The Twin circular configuration pressure more slowly rises to ambient conditions than the Exchange Inlet.

The upper surface pressure lines are shown in Figure 58, The Exchange Inlet does not show a significant drop in pressure at the RNEP where choking is desired 


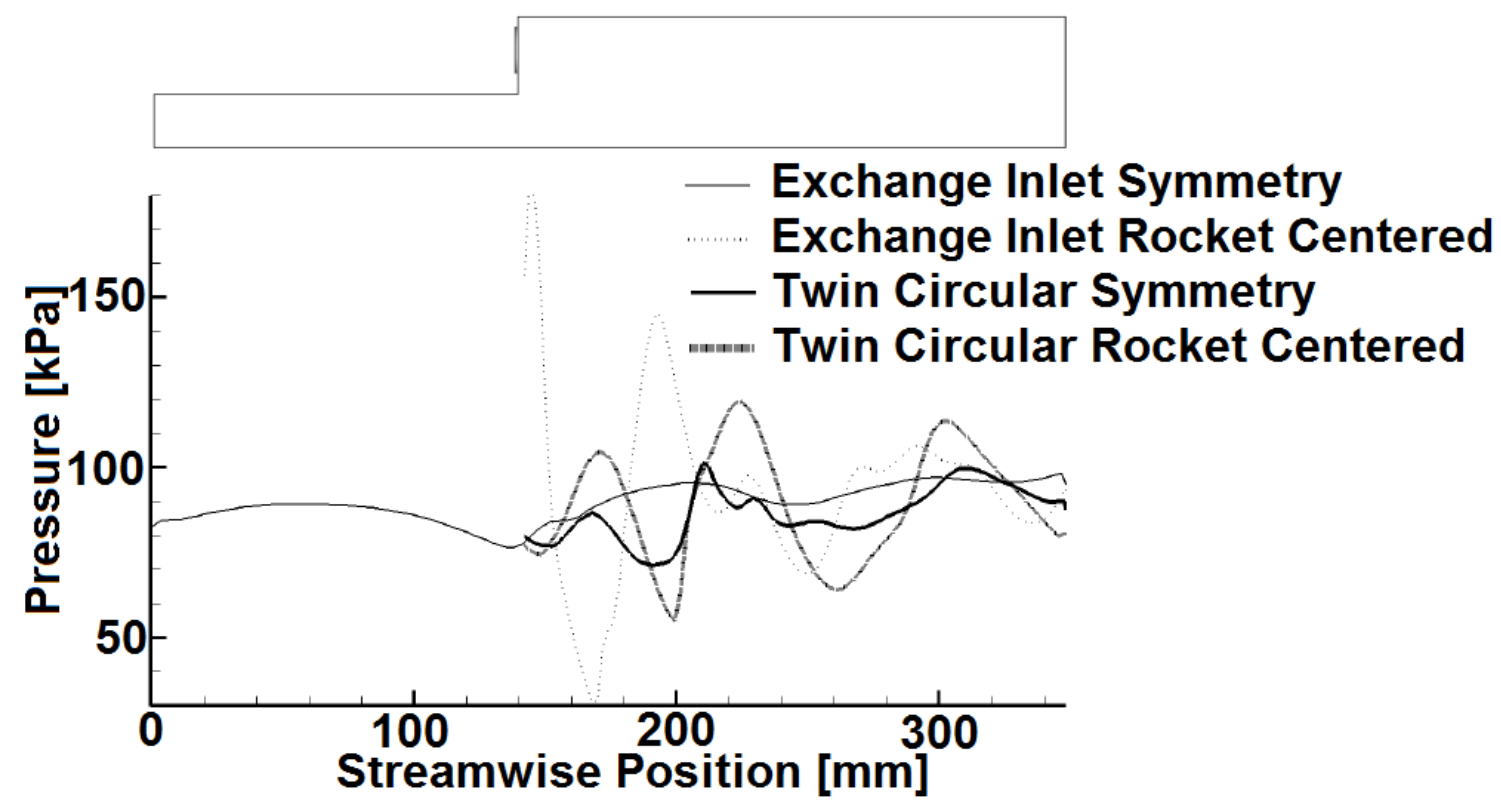

Figure 58: Upper Surface High Pressure Distribution for Short Engine configuration

along the centerline indicating less accelerated flow. It is seen that in the Twin circular symmetry line that the Twin circular configuration experiences some pressure changes as a result of the shock train while the Exchange Inlet does not. The rocket centered lines for the Exchange Inlet show greater peaks and minimums than for the Twin circular configuration. In both flows along the rocket centered line, the flow still shows oscillations in pressure until the EEP indicating that the shock train continues through the EEP.

\section{RNEP}

Figure 59 (a) shows the Mach Distribution of the high pressure Exchange Inlet case. The entrainment tubes do show some choked flow, however the entrained air area only reaches a maximum Mach number of 0.7. The long configuration for the Exchange Inlet has a maximum Mach number of 0.82 which is $14.6 \%$ greater so the short configuration does not perform as well as the long configuration. As was predicted by the pressure plots, the flow along the lower surface is evenly accelerated. The flow 


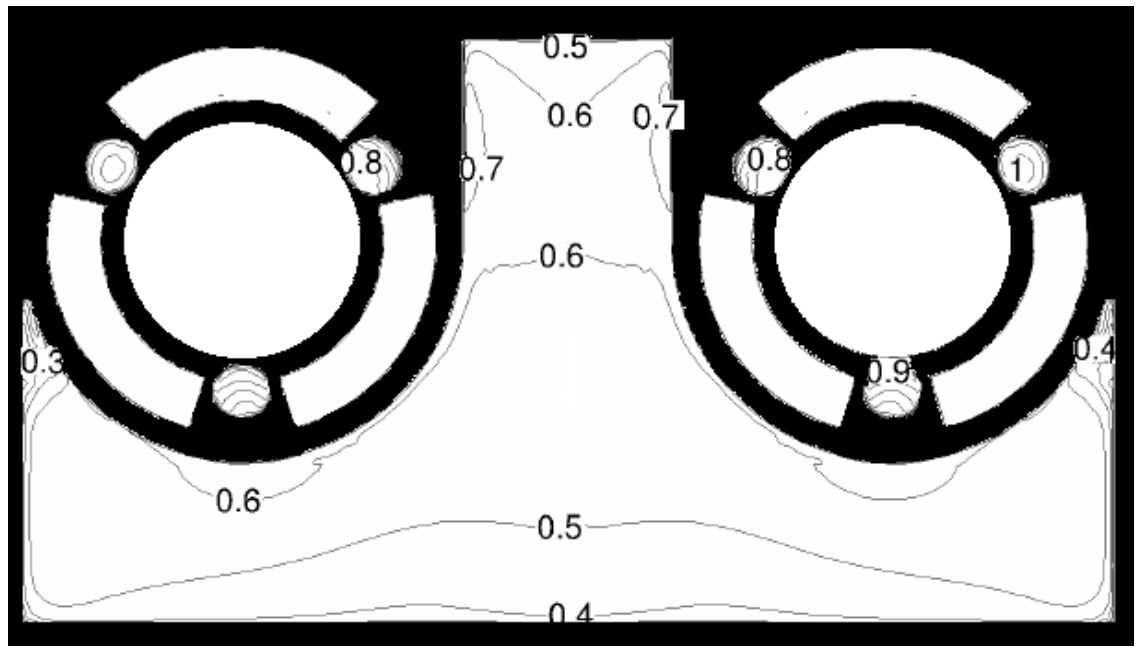

(a) Exchange Inlet

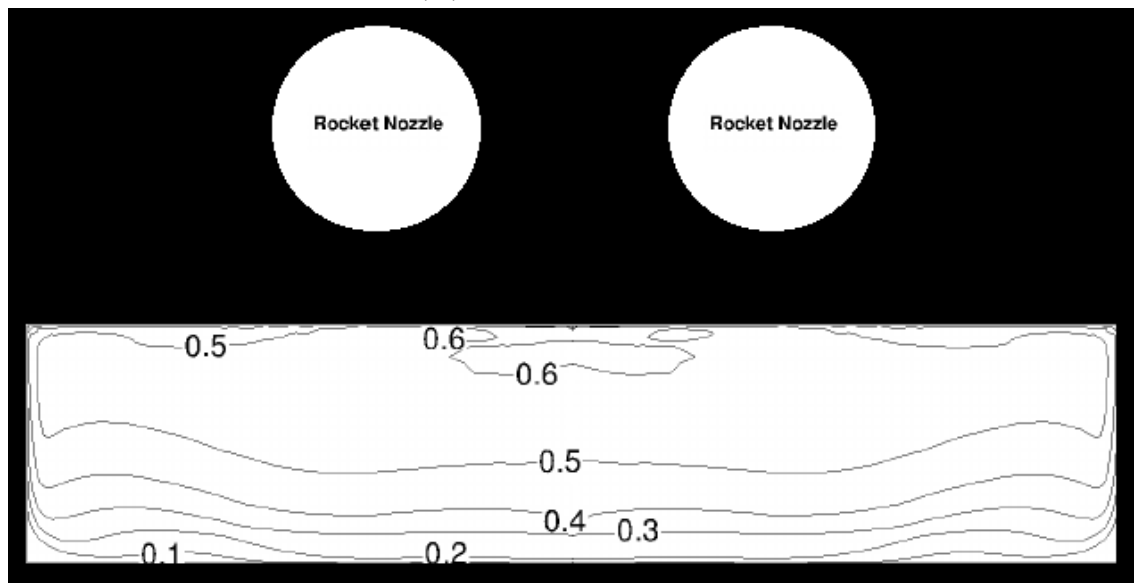

(b) Twin Circular

Figure 59: Short Configuration High Rocket Chamber Pressure Mach Distributions at RNEP

between the two rocket nozzles along the centerline shows the most accelerated flow.

In the RNEP shown in Figure 59 (b) of the Twin circular configuration there is no evident sign of choking. The entrained air accelerates to Mach 0.61 at the maximum value centered underneath both nozzles. The long configuration attains a significantly higher maximum Mach number of 0.9 in the RNEP which is $32.2 \%$ greater. Across all the Twin configuration simulations we see the flow accelerated along the upper surface of the entrainment duct near the centerline. The flow along 
the lower surface is also evenly accelerated in the Twin circular case. A much smaller area of the Twin circular entrained air is greater than Mach 0.5 than in the Exchange Inlet. The Exchange Inlet is able to accelerate much more of the flow. From Figures 57 and 58 the entrained air mass flows and entrainment ratio are determined. The entrained air mass flow of the Exchange Inlet is $0.211 \mathrm{~kg} / \mathrm{s}$ and then entrainment ratio is 0.347 while for the Twin circular configuration these values are $0.144 \mathrm{~kg} / \mathrm{s}$ and 0.306 respectively.

\section{EEP}

The Mach distribution is shown in Figure 60 (a) for the Exchange Inlet EEP. Near the center of the lower wall some recirculation exists causing the saddle shape. This recirculation slows the flow nearby. The peak Mach number at this plane is 1.51 . The sonic flow is able to penetrate almost to the middle of the Engine outlet. The two rocket cores are still evident in this plane along the outer surfaces. The average Mach number is 0.816. Compared to the ESP of the long configuration (where the ESP would be the at the same streamwise length of $\mathrm{x}=350 \mathrm{~mm}$ ), the long configuration has a peak and average Mach value of 1.9 and 1.23 respectively which is $20.5 \%$ and $33.7 \%$ respectively greater than the short configuration. This is a result of the EEP in the short configuration having a back pressure of atmospheric conditions whereas in the long configuration this same condition isn't reached until after the additional length of the expanding duct.

The Mach distribution plane shown in Figure 60 (b) displays the EEP of the Twin circular configuration. The peak Mach number is 1.92 compared to the long configuration of 1.8. The plane shows the rocket core has not dissipated very well by the exit. Over half of the outbound plane remains under Mach 0.5. The average Mach number of the exit plane is 0.700, lower than the long configuration ESP at 0.97. The peak Mach value is $6.66 \%$ greater in the short configuration but the average is $30 \%$ 


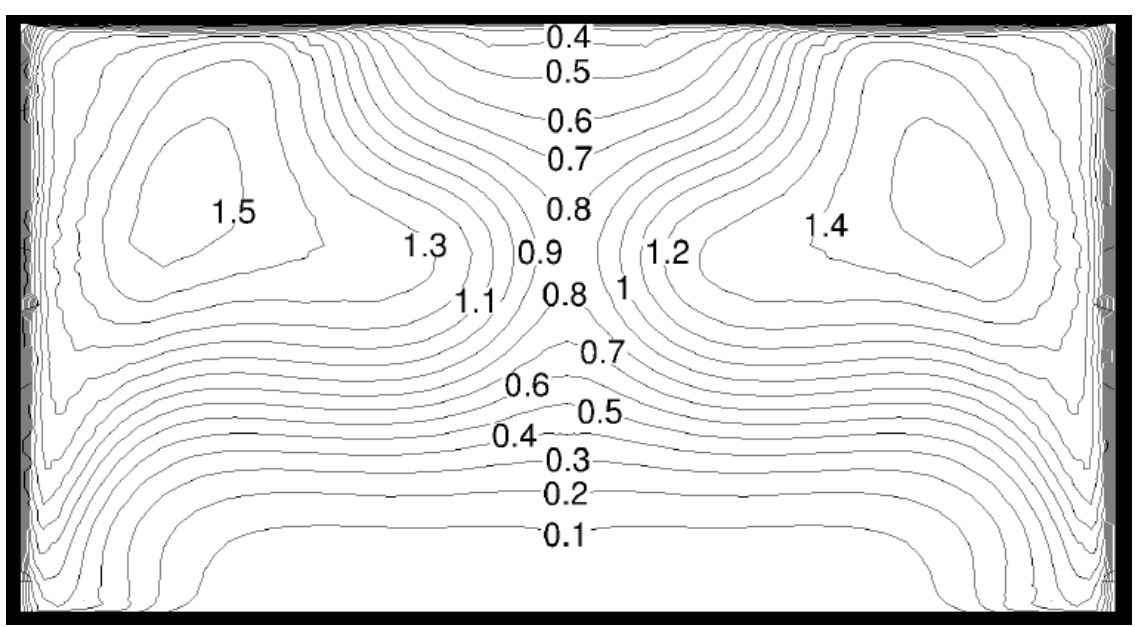

(a) Exchange Inlet

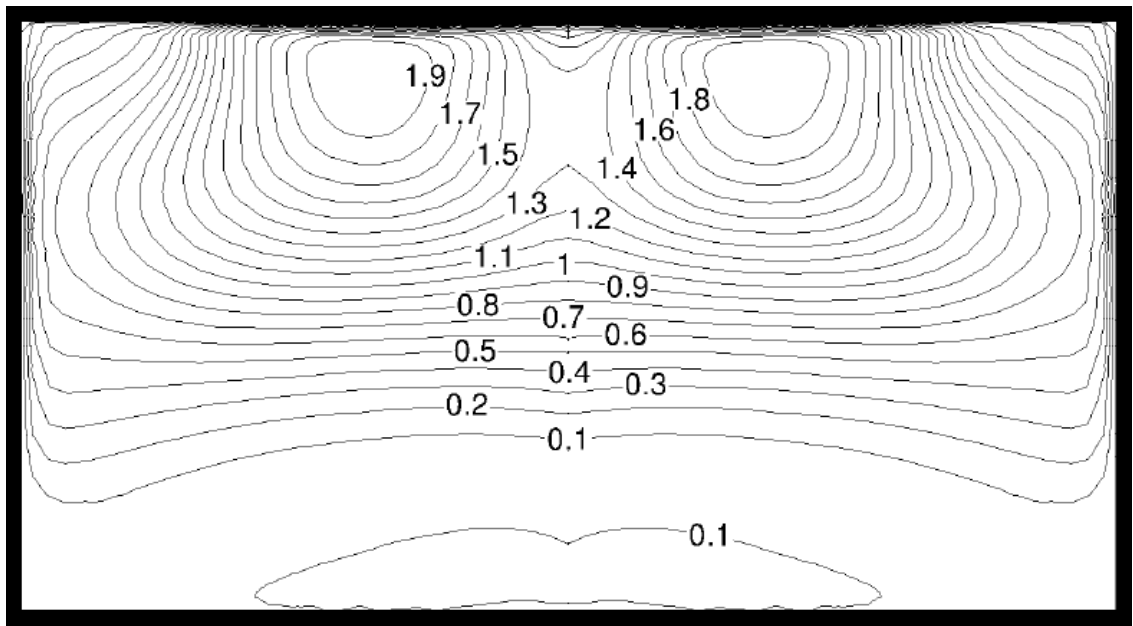

(b) Twin Circular

Figure 60: Short Configuration High Rocket Chamber Pressure Mach Distribution at EEP 
lower.

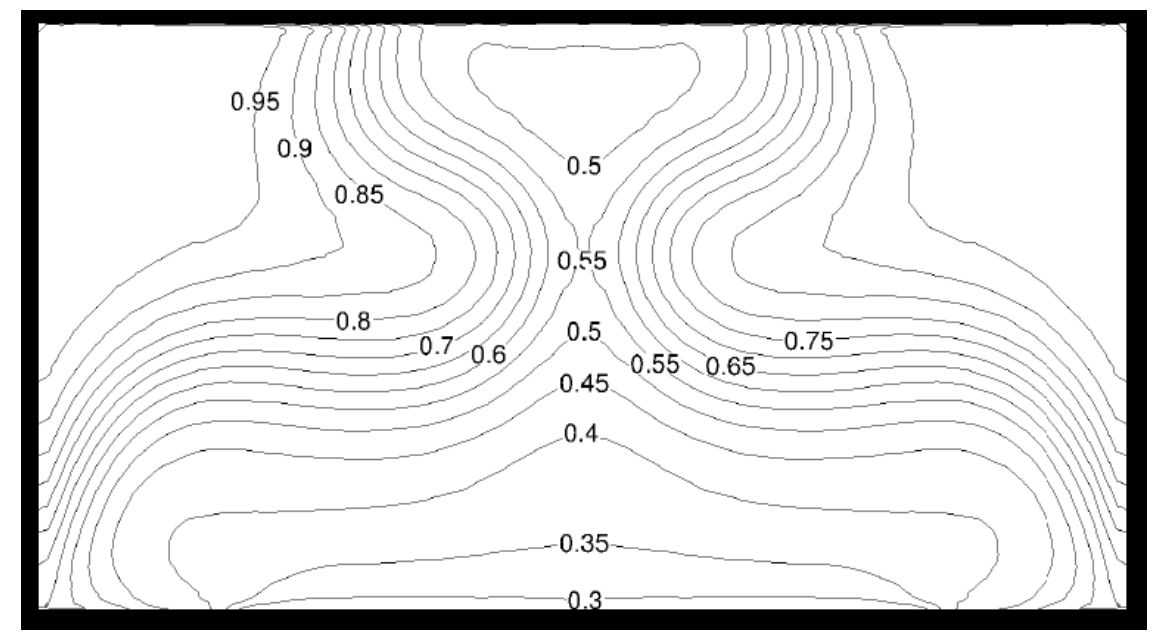

(a) Exchange Inlet

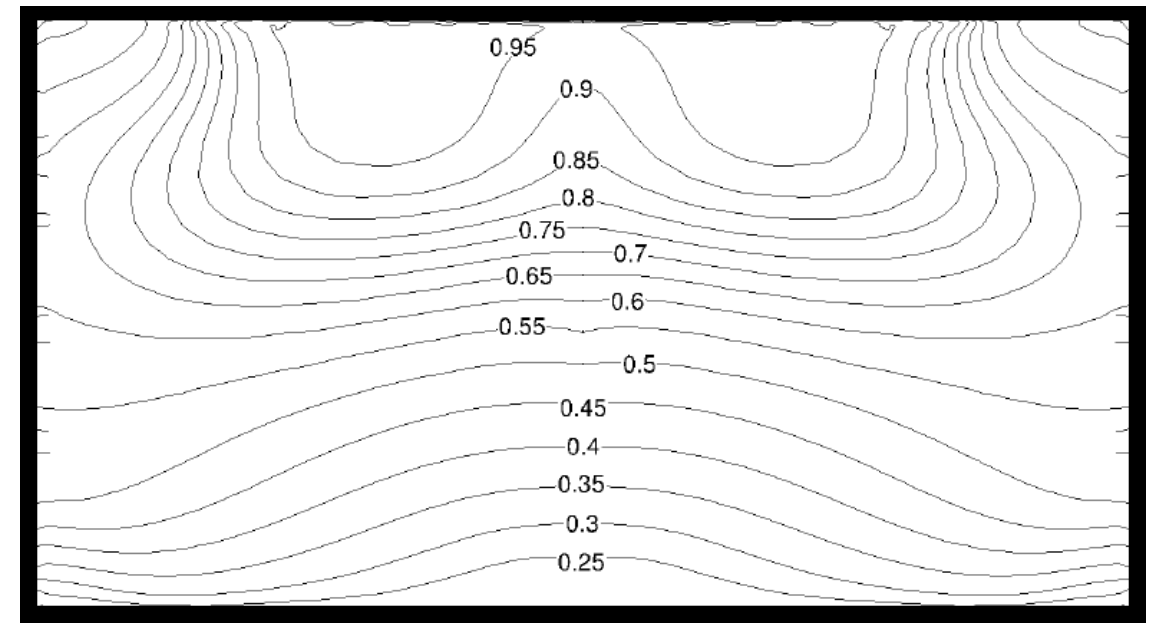

(b) Twin Circular

Figure 61: Short Configuration High Rocket Chamber Pressure $\mathrm{O}_{2}$ Partial Density at EEP

Figure 61 (b) shows the $\mathrm{O}_{2}$ partial density at the EEP of the Exchange Inlet. The recirculation mentioned prior tends to reduce the $\mathrm{O}_{2}$ partial density on the lower surface by suctioning air from the free stream. In the short configuration, a peak $\mathrm{O}_{2}$ partial density of $98 \%$ is found in the corners. This indicates that almost no mixing is occurring in these locations. Close to the centerline the flow is more mixed as $\mathrm{O}_{2}$ partial density tends to be lower. The average $\mathrm{O}_{2}$ partial density is $66.6 \%$. 
The $\mathrm{O}_{2}$ partial density for the Twin circular configuration is shown in Figure 61 (b). Similarly to the Exchange Inlet, peak values of $98 \%$ are seen where the rocket core still is present. The recirculation along the lower surface causes the $23 \%$ minimum of $\mathrm{O}_{2}$ partial density. The average $\mathrm{O}_{2}$ partial density is $61.3 \%$.

\section{Streamwise Planes}

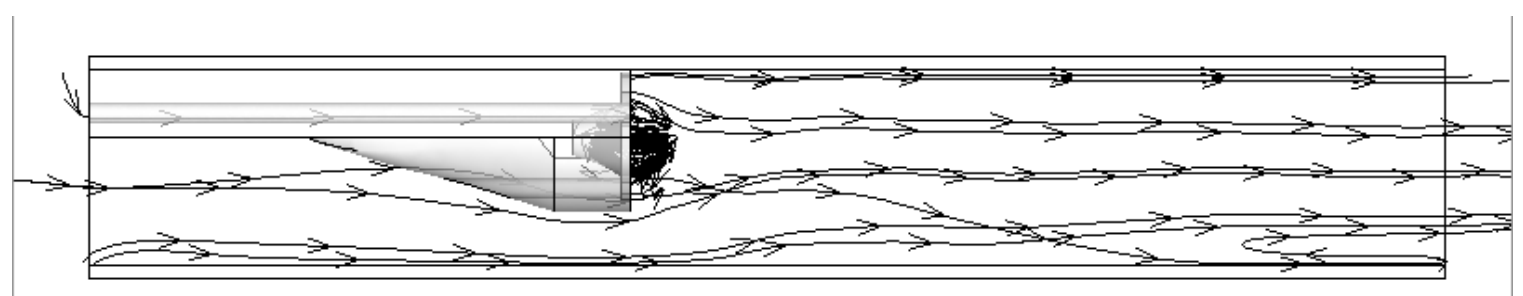

Figure 62: Exchange Inlet Short configuration Stream Trace

The stream trace of Figure 62 shows that there is some evidence of recirculation however minimal. The centerline has a minor amount of recirculation which may be the cause of the difference seen at the EEP.

The stream trace from Figure 63 clearly shows the recirculation in the engine. The recirculation of the Twin circular configuration is significantly more dominant than in the Exchange Inlet showing inefficiencies in designs comparatively.

Figure 64 shows the streamwise $\mathrm{O}_{2}$ partial density of the Exchange Inlet. The $\mathrm{O}_{2}$ plane shows the degree of mixing occurring within the short straight duct. The

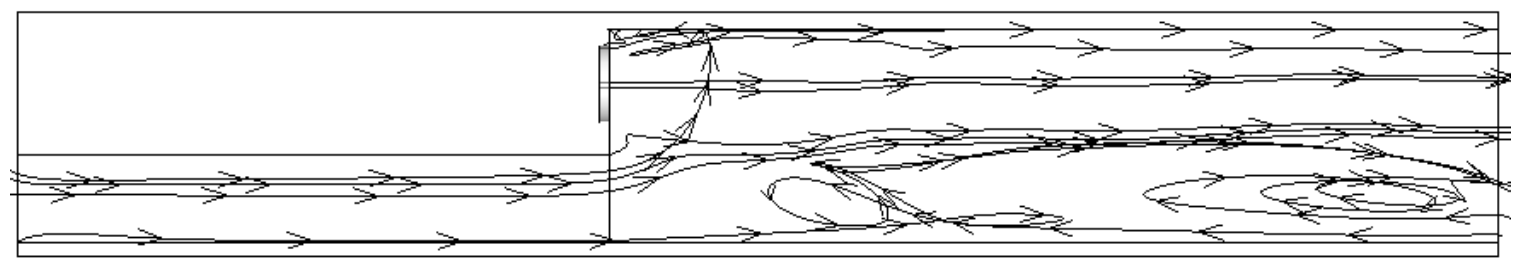

Figure 63: Twin Short Engine configuration Stream Trace 


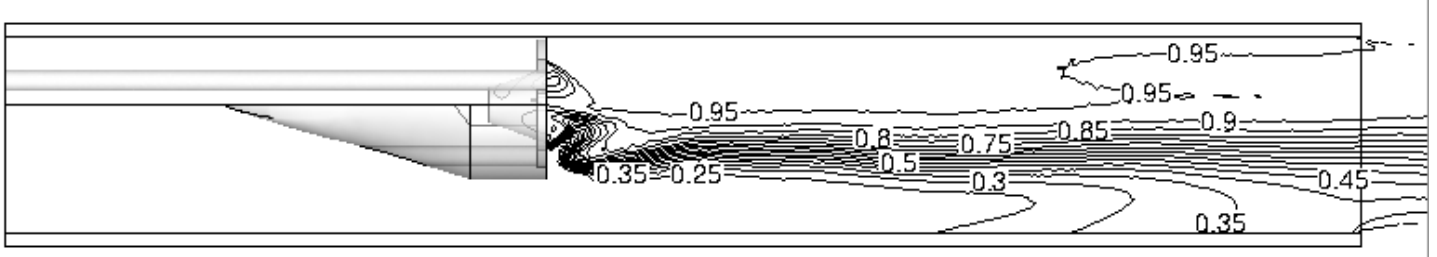

Figure 64: Exchange Inlet Short configuration $\mathrm{O}_{2}$ Partial Density along rocket flow plane

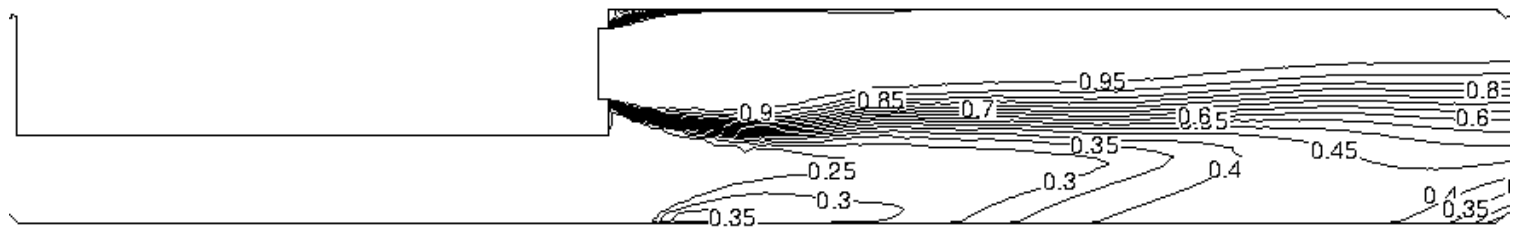

Figure 65: Twin Short configuration $\mathrm{O}_{2}$ Partial Density along Rocket Plane

entrained air moves farther along the lower surface than is seen in the Twin configuration.

Figure 65 shows the $\mathrm{O}_{2}$ partial density inside the Twin circular configuration. The rocket plane shows that some recirculation early in the engine causes the $\mathrm{O}_{2}$ partial density to be increased almost directly under the rocket stream. The flow remains largely together and concentrated along the upper surface as was seen in the low pressure short configuration. The results of the recirculation can be seen around the outlet plane where flow partial density starts to decrease.

A summary of the entrainment properties is given in Table 9.

\subsection{Additional Experimental Data}

Additional experimental data was taken for the short configurations to examine the consistency of results between the experiment and numerical analysis. 


\begin{tabular}{|c|c|c|c|c|}
\hline & \multicolumn{2}{|c|}{ Short Configuration } & \multicolumn{2}{c|}{ Long Configuration } \\
\hline Configuration & Twin circular & Exchange Inlet & Twin circular & Exchange Inlet \\
\hline Mass Flow Air $\left(\dot{m}_{\text {air }}\right)$ & 0.144 & 0.211 & 0.186 & 0.244 \\
\hline Mass Flow Rocket $\left(\dot{m}_{r}\right)$ & 0.470 & 0.608 & 0.485 & 0.596 \\
\hline Entrainment Ratio & 0.306 & 0.347 & 0.384 & 0.409 \\
\hline $\bar{M}$ Exit Plane & 0.700 & 0.816 & 0.681 & 0.703 \\
\hline $\bar{O}_{2}$ Exit Plane & $61.3 \%$ & $66.6 \%$ & $64.1 \%$ & $62.8 \%$ \\
\hline
\end{tabular}

Table 9: Summary Of High Pressure Short Configuration Results

The lower surface pressure plots of the Exchange Inlet and Twin circular configuration are shown in Figures 66 and 67.

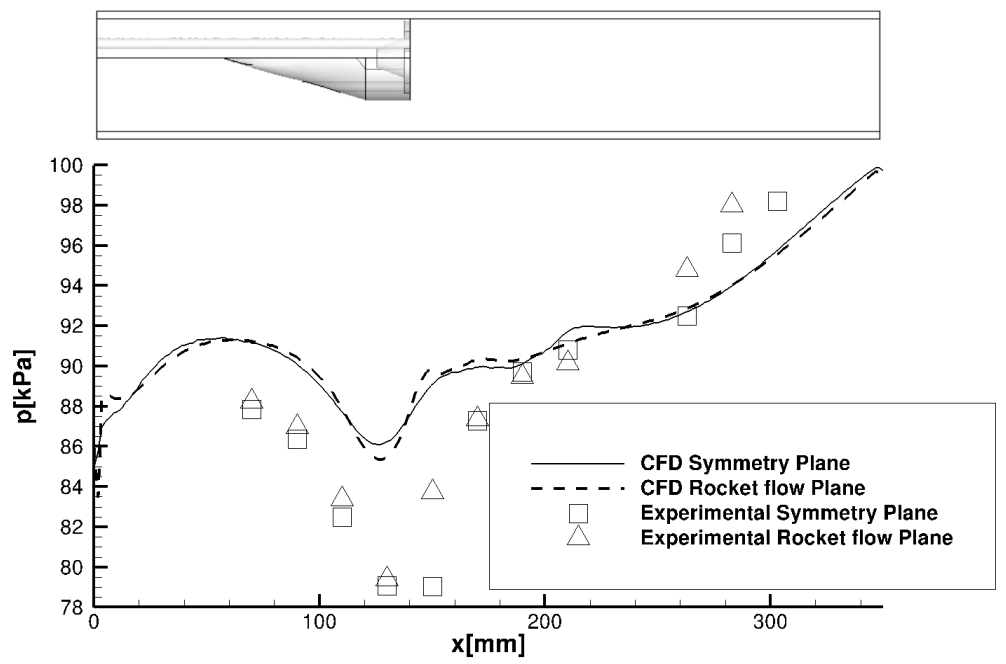

Figure 66: Exchange Inlet Short configuration Lower surface low pressure Experimental VS Numerical data

From these two figures, it is seen that accuracy of the simulations is not maintained when reducing the length of the engine. In the validation case (long configuration), both results were within $6 \%$ of experimental data and pressure values were greater in the numerical simulation than the experiment captured. For the short case the experimental data differs as much as $25 \%$ with numerical analysis pressure values along the shown planes for the Twin circular configuration to be lower than the 


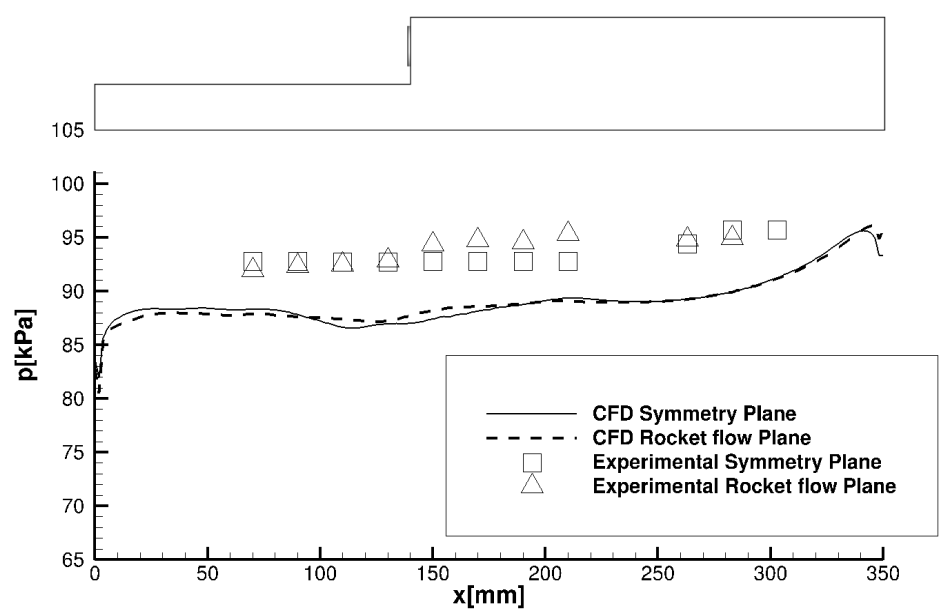

Figure 67: Twin circular Short configuration Lower surface low pressure Experimental VS Numerical data

experimental value. The numerical analysis also tends to over predict the entrainment performance of the Twin circular configuration while the Exchange Inlet is under predicted for the short engine configurations.

For the short configuration, the numerical vs. experimental results do not follow the same trend as the long configuration. While still capturing a general trend that the Exchange Inlet is able to have increased entrainment over the Twin circular configuration, the difference seen numerically is not as significant in the short configuration. 


\section{Chapter 5}

\section{Conclusions}

A detailed analysis of two nozzle configurations both within two engine configurations is conducted for varying rocket chamber pressures. The two nozzle configurations are the Exchange Inlet which has an annular exhaust where rocket flow expands out of three sections and a circular nozzle where rocket flow is expanded more traditionally. The two engine configurations that these nozzles are placed in are a long configuration measuring $725 \mathrm{~mm}$ in length and a short configuration measuring $350 \mathrm{~mm}$ in length. In the short configuration the expanding section from the long configuration is removed. The Exchange Inlet Configuration is found to produce higher entrainment ratios than the Twin circular configuration in a Rocket Based Combined cycle engine design.

The long engine configuration is examined at two pressure settings for the two nozzles and the Exchange Inlet is found to perform better. The Exchange Inlet achieves higher entrainment ratios, higher average Mach numbers and Oxygen concentrations than the Twin Circular configuration. The low pressure simulation of the Exchange Inlet is found to have a shock train that dissipates quickly and aids in mixing. The Twin configuration shock train does not dissipate as quickly and a thin area of recirculation exists along the lower surface at the exit plane. The Twin Circular configuration maintains its rocket core through the flow much more than the Exchange Inlet which is less desirable as a fully mixed Engine Exit plane is the goal. 
The high pressure long configurations similarly show that the Exchange Inlet configuration has higher entrainment ratios, Mach numbers, and oxygen concentrations. While neither flow choke at the aerodynamic choke, the Exchange Inlet does reach a Fabri choke. The air flow through both engines is found to be near to the low pressure configuration indicating that a maximum amount of air flow is reached. The pressure lines of the high pressure configurations show a longer shock train from both configurations but the Exchange Inlet's shock train again dissipates quicker. The outbound planes show that the Exchange inlet has more mixed flow in terms of oxygen concentration and Mach number than the Twin circular configuration.

The short configurations are considered in order to examine the nozzle performance more directly. Without the expanding section of the long configuration, atmospheric conditions constrain the engine by having a higher back pressure at a lower mixing length. It does however also show that the Exchange Inlet has higher entrainment ratios however not as pronounced as with the long configurations. Additionally, recirculation is significantly more evident in the short configurations than it is in the long configurations. In the low pressure short configuration the rocket cores are still seen at the Engine Exit plane and the flow shock structures have not dissipated as well as the long configurations.

The short high pressure configuration shows an even greater recirculation in the Twin Circular configuration than the low pressure short configuration. In the high pressure case the Exchange Inlet has a higher entrainment ratio again.

Additional experimental information showed that the predicted simulations for the short configurations did not capture pressure effects as accurately as the long configurations.

The Exchange Inlet has increased performance in terms of entrainment, Mach and oxygen concentrations in all cases. 


\section{List of References}

[1] D. Clary. Rocket Man: Robert H. Goddard and the Birth of the Space Age. Hachette Books. ISBN 9781401398330 (2003).

[2] J. Pelton and A. Bukley. The Farthest Shore: A 21st Century Guide to Space. Apogee Books space series. Collector's Guide Publishing, Incorporated. ISBN 9781926592077 (2010).

[3] B. Dunbar. FY 2014 Budget Proposal NASA. http://www.nasa.gov/content/ fy-2014-budget-proposal/\#.VKqroCvF8Vv ("2014 (accessed December 20, 2014)").

[4] S. Rogers. NASA Budget;US Spending on space travel since 1958. http://www.theguardian.com/news/datablog/2010/feb/01/ nasa-budgets-us-spending-space-travel (2010 (Accessed January 15 2015)).

[5] T. Benson. NASA; Specific Impulse. http://www.grc.nasa.gov/WWW/k-12/ airplane/specimp.html (2014 (Accessed January 15 2015)).

[6] B. C. Edwards. "Design and deployment of a space elevator." Acta Astronautica 47(10), 735-744 (2000).

[7] I. R. McNab. "Launch to space with an electromagnetic railgun." Magnetics, IEEE Transactions on 39(1), 295-304 (2003).

[8] W. J. Escher, B. J. Flornes, et al. "A study of composite propulsion systems for advanced launch vehicle applications." Contract NAS7-37\%. The Marquardt Corporation: Van Nuys, California 1, 7 (1966).

[9] W. J. Escher. "Synerjet for earth/orbit propulsion: Revisiting the 1966 nasa/marquardt composite (airbreathing/rocket) propulsion system study." Technical report, SAE Technical Paper (1985). 
[10] A. Murzionak. Rocket Based Combined Cycle Exchange Inlet Performance Estimation at Supersonic Speeds. Master's thesis, Carleton University (2010).

[11] R. Daines and C. Segal. "Combined rocket and airbreathing propulsion systems for space-launch applications." Journal of Propulsion and Power 14(5), 605-612 (1998).

[12] J. C. Mankins. "Highly reusable space transportation: Advanced concepts and the opening of the space frontier." Acta astronautica 51(10), 727-742 (2002).

[13] R. S. Fry. "A century of ramjet propulsion technology evolution." Journal of propulsion and power 20(1), 27-58 (2004).

[14] M. Smart. "Scramjets." Aeronautical Journal 111(1124), 605-620 (2007).

[15] P. T. Harsha, L. C. Keel, A. Castrogiovanni, and R. T. Sherrill. "X-43a vehicle design and manufacture." In "28th JANNAF Airbreathing Propulsion Subcommittee Meeting. Charleston, SC. June," pages 13-17 (2005).

[16] C. E. Kelly. Boeing: Boeing X-51A Waverider Breaks Record in First Flight. http://boeing . mediaroom . com/index . php?s=20295\&item=1227 (2010 (Accessed January 15 2015)).

[17] J. Keenan and E. Neumann. "A simple air ejector." Journal of Applied Mechanics 9(2), A75-A81 (1942).

[18] J. Fabri and J. Paulon. Theory and experiments on supersonic air-to-air ejectors. National Advisory Committee for Aeronautics (1958).

[19] J. Fabri and R. Siestrunck. "Supersonic air ejectors." Advances in applied mechanics 5, 1-34 (1958).

[20] A. Addy and W. Chow. "Interaction between primary and secondary streams of supersonic ejector systems and their performance characteristics." AIAA Journal 2(4), 686-695 (1964).

[21] A. Addy, J. C. Dutton, and C. Mikkelsen. "Supersonic ejector-diffuser theory and experiments." Technical report, DTIC Document (1982).

[22] D. Papamoschou. "Analysis of partially mixed supersonic ejector." Journal of propulsion and power 12(4), 736-741 (1996).

[23] G. Emanuel. "Comparison of one-dimensional solutions with fabri theory for ejectors." Acta Mechanica 44(3-4), 187-200 (1982). 
[24] B. Huang, J. Chang, C. Wang, and V. Petrenko. "A 1-d analysis of ejector performance." International Journal of Refrigeration 22(5), 354-364 (1999).

[25] S. Han and J. Tomes. "A numerical study of marquardt's ejector-scramjet test engine." AIAA Paper 3606, 2002 (2002).

[26] J. G. del Valle, J. M. S. Jabardo, F. C. Ruiz, and J. S. J. Alonso. "A one dimensional model for the determination of an ejector entrainment ratio." international journal of refrigeration 35(4), 772-784 (2012).

[27] Y. Bartosiewicz, Z. Aidoun, P. Desevaux, and Y. Mercadier. "Numerical and experimental investigations on supersonic ejectors." International Journal of Heat and Fluid Flow 26(1), 56-70 (2005).

[28] Y. Zhu, W. Cai, C. Wen, and Y. Li. "Numerical investigation of geometry parameters for design of high performance ejectors." Applied Thermal Engineering 29(5), 898-905 (2009).

[29] S. Varga, A. C. Oliveira, and B. Diaconu. "Influence of geometrical factors on steam ejector performance-a numerical assessment." International journal of refrigeration 32(7), 1694-1701 (2009).

[30] S. Varga, A. C. Oliveira, X. Ma, S. A. Omer, W. Zhang, and S. B. Riffat. "Experimental and numerical analysis of a variable area ratio steam ejector." international journal of refrigeration 34(7), 1668-1675 (2011).

[31] M. N. Jahingir and Z. Huque. "Design optimization of rocket-based combinedcycle inlet/ejector system." Journal of propulsion and power 21(4), 650-655 (2005).

[32] E. Petersen, V. Roan, and J. Pfahler. "Experimental investigation of supersonicprimary dissimilar-fluid ejectors." In "Joint Propulsion Conference and Exhibit," volume 1 (1992).

[33] F. Dijkstra, A. Maree, M. Caporicci, and H. Immich. "Experimental investigation of the thrust enhancement potential of ejector rockets." In "Proc. 33rd Joint Propulsion Conference and Exhibit, Seattle, WA," (1997).

[34] J.-H. Lee, V. S. Kumar, H. Kim, C. Bo-Gyu, K. Kim, and T. Setoguchi. "Studies on flow characteristics of variable ejector systems." In "Proceedings of 42nd AIAA/ASME/SAE/ASEE Joint Propulsion Conference \& Exhibit," (2006). 
[35] D. Lineberry, N. Smith, D. Landrum, and C. Hawk. "Characterization of cold flow non-axisymmetric ejectors." AIAA Paper 5231, 2003 (2003).

[36] M. Balasubramanyam, D. B. Landrum, C.-P. Chen, and D. Lineberry. "Numerical investigation of cold flow non-axisymmetric ejectors." In "43 rd AIAA Aerospace Sciences Meeting and Exhibit," page 2005 (2005).

[37] D. Lineberry and B. Landrum. "Effect of multiple nozzles on asymmetric ejector performance." In "Proceedings of the 41st AIAA/ASME/SAE/ASEE Joint Propulsion Conference and Exhibit, Report No. AIAA2005-4283," (2005).

[38] M. Balasubramanyam, D. Lineberry, C. Chen, and D. Landrum. "Experimental and numerical investigation of a non-axisymmetric strut based ejector." International Journal of Hypersonics 1(3), 181-198 (2010).

[39] H. D. P. Scott R. Thomas and C. J. Trefny. Strutjet engine which integrates a rocket into a ramjet/scramjet propulsion system. http://commons.wikimedia. org/wiki/File:Air_augmented_rocket_en.svg (1997 (Accessed February 8 2015).

[40] Z. Samitha, D. Davis, and P. Balachandran. "Computational study on supersonic mixing using clover nozzle." In "AIAA Aerospace Sciences Meeting and Exhibit," pages 2007-839 (2007).

[41] S. M. Rao and G. Jagadeesh. "Novel supersonic nozzles for mixing enhancement in supersonic ejectors." Applied Thermal Engineering 71(1), 62-71 (2014).

[42] G. Hagemann, H. Immich, T. V. Nguyen, and G. E. Dumnov. "Advanced rocket nozzles." Journal of Propulsion and Power 14(5), 620-634 (1998).

[43] D. J. Cerantola and J. Etele. "A nozzle concept to entrain atmospheric air for ejector operation." In "14th AIAA/AHI Space Planes and Hypersonic Systems and Technologies Conference," (2006).

[44] J. Etele, J. Sislian, and B. Parent. "Effect of rocket exhaust configurations on ejector performance in rbcc engines." Journal of propulsion and power 21(4), 656-666 (2005).

[45] T. S. C. Yuen. Simulation of a Rocket Base Combined Cycle Exchange Inlet at Subsonic Conditions. Ph.D. thesis, Carleton University Ottawa (2012). 
[46] J. Etele, S. Hasegawa, and S. Ueda. "Experimental investigation of an alternative rocket configuration for rocket-based combined cycle engines." Journal of Propulsion and Power 30(4), 944-951 (2014).

[47] R. Courtland. "The status of moores law: Its complicated." IEEE Spectrum, Oct (2013).

[48] C. A. Mack. "Fifty years of moore's law." Semiconductor Manufacturing, IEEE Transactions on 24(2), 202-207 (2011).

[49] H. K. Versteeg and W. Malalasekera. An introduction to computational fluid dynamics: the finite volume method. Pearson Education (2007).

[50] T. A. Oliver. "Favre-averaged navier-stokes and turbulence model equation documentation." Technical report, Technical report, Predictive Engineering and Computational Sciences, University of Texas at Austin (2011).

[51] F. R. Menter. "Two-equation eddy-viscosity turbulence models for engineering applications." AIAA journal 32(8), 1598-1605 (1994).

[52] K. Nakahashi, D. Sharov, S. Kano, and M. Kodera. "Applications of unstructured hybrid grid method to high-reynolds number viscous flows." International journal for numerical methods in fluids 31(1), 97-111 (1999).

[53] M. Kodera, T. Sunami, and F. Scheel. "Numerical study on the supersonic mixing enhancement using streamwise vortices." AIAA paper 5117, 2002 (2002).

[54] M. Kodera, K. Nakahashi, T. Hiraiwa, T. Kanda, and T. Mitani. "Scramjet inlet flow computations by hybrid grid method." AIAA paper pages 98-0962 (1998).

[55] R. J. LeVeque. Finite volume methods for hyperbolic problems, volume 31. Cambridge university press (2002).

[56] F. M. White. "Fluid mechanics (6th international edition)." (2007). 